Tiia Ojanperä

\title{
CROSS-LAYER OPTIMIZED VIDEO STREAMING IN HETEROGENEOUS WIRELESS NETWORKS
}

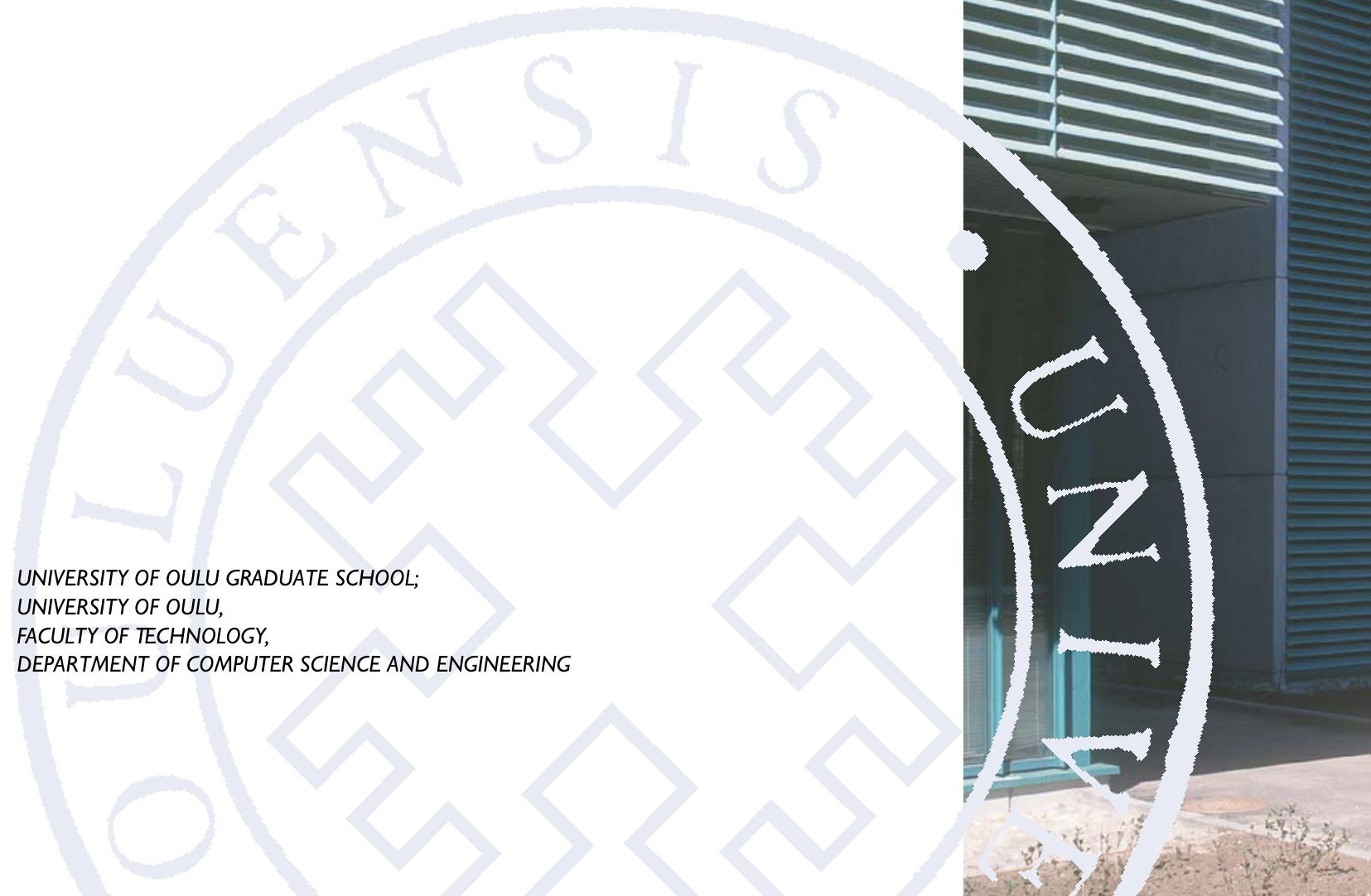



ACTA UNIVERSITATIS OULUENSIS

C Technica 453

TIIA OJANPERÄ

CROSS-LAYER OPTIMIZED VIDEO STREAMING IN HETEROGENEOUS WIRELESS NETWORKS

Academic dissertation to be presented with the assent of the Doctoral Training Committee of Technology and Natural Sciences of the University of Oulu for public defence in OP-sali (Auditorium LI0), Linnanmaa, on 19 June 2013 , at 12 noon 
Copyright (C) 2013

Acta Univ. Oul. C 453, 2013

Supervised by

Professor Timo Ojala

Reviewed by

Professor Jörg Ott

Professor Christer Åhlund

ISBN 978-952-62-0I50-4 (Paperback)

ISBN 978-952-62-0I5I-I (PDF)

ISSN 0355-32 I 3 (Printed)

ISSN 1796-2226 (Online)

Cover Design

Raimo Ahonen

JUVENES PRINT

TAMPERE 20I3 


\title{
Ojanperä, Tiia, Cross-layer optimized video streaming in heterogeneous wireless networks
}

University of Oulu Graduate School; University of Oulu, Faculty of Technology, Department of Computer Science and Engineering, P.O. Box 4500, FI-90014 University of Oulu, Finland

Acta Univ. Oul. C 453, 2013

Oulu, Finland

\begin{abstract}
This dissertation studies the impact of heterogeneous wireless networks on the design and implementation of mobile video streaming services. The aim is to enable Quality of Service (QoS) sensitive video streaming services to take full advantage of the access diversity of heterogeneous networks in order to optimize their operation in terms of quality and efficiency of network resource usage. This nevertheless requires support beyond the layered communication architecture of today's Internet.

The thesis proposes an architecture for end-to-end cross-layer signaling and control for video streaming systems. The architecture supports extensive context information transfer in heterogeneous networks; thus, enabling the efficient management of video stream adaptation and user terminal mobility in a diverse and dynamic network environment. This thesis also studies and proposes cross-layer enhancements for adaptive video streaming and mobility management functions enabled by the cross-layer architecture. These include cross-layer video adaptation, congestion-triggered handovers, and concurrent utilization of multiple access networks in the video stream transport. For the video adaptation and multipath transmission, the flexible adaptation and transmission capabilities of the novel scalable video coding technology are used. Regarding the mobility management, the proposed solutions essentially enhance the handover decision-making of the Mobile IP protocol to better support QoS-sensitive video streaming. Finally, the thesis takes a holistic view on the application adaptation and mobility management, and proposes a solution for coordinated control of these two operations in order to achieve end-toend optimization.

The resulting mobile video streaming system architecture and the cross-layer control algorithms are evaluated using network simulations and real prototypes. Based on the results, the proposed mechanisms can be seen to be viable solutions for improving video streaming performance in heterogeneous wireless networks. They require changes in the communication end-points and the access network but support gradual deployment. This allows service providers and operators to select only a subset of the proposed mechanisms for implementation. The results also support the need for cross-layer signaling and control in facilitating efficient management and utilization of heterogeneous wireless networks.
\end{abstract}

Keywords: cross-layer information, decision-making, handover, heterogeneous netwoks, mobility management, multi-homing, scalable video coding, video adaptation 



\section{Ojanperä, Tiia, Videonsiirron optimointi langattomissa verkoissa protokolla- kerrosten välistä tietoa hyödyntäen}

Oulun yliopiston tutkijakoulu; Oulun yliopisto, Teknillinen tiedekunta, Tietotekniikan osasto, PL 4500, 90014 Oulun yliopisto

Acta Univ. Oul. C 453, 2013

Oulu

\section{Tiivistelmä}

Väitöskirja tutkii langattomien ja heterogeenisen verkkoympäristöjen vaikutusta erityisesti mobiilikäyttöön suunnattuihin suoratoistovideopalveluihin (streaming). Työn tavoitteena on löytää keino optimoida verkkoyhteyden palvelunlaadulle (QoS) herkän suoratoistovideon toiminta sekä videopalvelun laadun että verkon tiedonsiirtokapasiteetin käytön osalta. Tämä tapahtuu mahdollistamalla heterogeenisten verkkojen tehokas käyttö suoratoistovideopalvelujen tapauksessa. Tavoitellut parannukset vaativat kuitenkin muutoksia nykyiseen kerroksittaiseen Internetarkkitehtuuriin.

Väitöskirjassa esitetään arkkitehtuuri protokollakerrosten välisen (cross-layer) tiedon välitykseen ja hyödyntämiseen suoratoistovideopalvelujen tiedonsiirron kontrolloinnissa. Arkkitehtuuria voidaan käyttää laaja-alaiseen kontekstitiedon välitykseen tietoverkoissa, mikä mahdollistaa tehokkaan videopalvelun adaptoinnin ja päätelaitteen liikkuvuudenhallinnan heterogeenisissa verkoissa, joissa palvelunlaatu vaihtelee. Väitöskirja myös ehdottaa erilaisia ratkaisuja videopalvelun adaptoinnin ja tiedonsiirron parantamiseksi arkkitehtuuria hyödyntämällä. Näihin lukeutuvat usealle protokollakerrokselle toteutettu videon adaptointi, verkkoyhteyden ruuhkautumiseen reagoiva yhteydensiirto sekä usean verkkoyhteyden samanaikainen käyttö videopalvelun tiedonsiirrossa. Videon adaptoinnissa ja siirrossa hyödynnetään uutta skaalautuvaa videonkoodausteknologiaa, joka mahdollistaa vaaditun, joustavan videobittivirran muokkauksen. Liikkuvuudenhallinnan osalta työssä keskitytään pääosin kehittämään Mobile IP -protokollan päätöksentekoa suoratoistovideopalvelujen tapauksessa. Lopuksi väitöskirjassa esitetään kokonaisvaltainen ja koordinoitu ratkaisu videopalvelun adaptoinnin sekä päätelaitteen liikkuvuuden hallintaan päästä päähän -optimoinnin saavuttamiseksi.

Tuloksena esitetyt järjestelmäarkkitehtuuri ja protokollakerrosten välistä tietoa käyttävät hallinta-algoritmit evaluoitiin simulaatioiden ja oikeiden prototyyppien avulla. Tulokset osoittavat, että ehdotettuja menetelmiä voidaan käyttää parantamaan suoratoistovideopalvelujen suorituskykyä heterogeenisissa verkoissa. Ratkaisut vaativat muutoksia verkko- ja palveluarkkitehtuureihin, mutta niiden asteittainen tai osittainen käyttöönotto on mahdollista. Tulokset osoittavat myös protokollakerrosten välisen tiedon tarpeellisuuden langattomien ja heterogeenisten verkkojen tehokkaassa käytössä.

Asiasanat: heterogeeniset verkot, liikkuvuudenhallinta, protokollakerrosten välinen tieto, päätöksenteko, skaalautuva videonkoodaus, videon adaptointi, yhteydensiirto 



\section{Preface}

I was introduced to the interesting world of heterogeneous wireless networks already a decade ago when starting my master's thesis work on the topic at MediaTeam Oulu. Back then, the topic enjoyed great interest within the research community, offering challenging problems in terms of developing novel mobility protocols and studying the impacts of inter-technology mobility on existing network infrastructures and operator business models. The first commercial implementations of Mobile IP were available although the technology was still under development. Based on the user study that I conducted for my M.Sc. thesis, it was clear that existing applications and transport protocols were lacking additional support to operate efficiently in heterogeneous networks. Yet, the idea of staying optimally connected to the Internet by exploiting the available access networks sounded appealing to the users. Thus, I was glad to return to the research topic after transferring to VTT Technical Research Centre of Finland in 2005 and becoming VTT's project manager in the Tekes MERCoNe project in 2006.

The constantly increasing popularity of accessing the Internet wirelessly has made the ability to use multiple access technologies and networks in supporting the generated data loads even more topical today than it was before. Wireless network operators always search for solutions that help them to ensure sufficient QoS for their customers' networked applications and services while minimizing the network operation costs. In this dissertation, I approach the problem in the viewpoint of one of the most demanding types of Internet services, that is, video streaming.

The contributions of this thesis resulted from several research projects that received funding from Tekes - the Finnish Funding Agency for Technology and Innovation, EU, Academy of Finland, VTT or industrial partners. In the context of utilizing the access diversity of heterogeneous multi-access networks in video transport, the work presented here was mainly conducted in the Tekes MERCoNe, EUREKA/Celtic SCALNET, and VTT's internal ITSE projects. On the other hand, the ability to adjust the video bitstream characteristics, such as throughput, efficiently to the available network capacity was considered within the frameworks of the EUREKA/Celtic SCALNET and EU FP7 ICT OPTIMIX. In addition, the Nordunet-3 ADIMUS acted as an umbrella project combining the results from the other projects in the preparation of this dissertation. My 
thesis has also been supported by scholarships granted by the foundations of TeliaSonera Finland and Tauno Tönning.

During the lengthy process of preparing my dissertation, I have been privileged to work with and receive guidance and support from several experts and other people, to whom I owe the deepest gratitude and whose contribution I want to acknowledge next.

I especially want to thank my supervisor Professor Timo Ojala for his continuous guidance and encouragement in my pursuit of both the M.Sc. and Ph.D. degrees as well as for his assistance when finalizing this dissertation. I also thank Professors Jörg Ott and Christer Åhlund for their thorough pre-examination and comments that helped me to improve the final version of the thesis.

I am most grateful to my colleague Mr. Jyrki Huusko for his support and guidance in compiling this dissertation over the years at VTT. I honestly believe I never would have finished this without his help. In addition, I thank Dr. Jussi Paakkari and Dr. Marko Jurvansuu for encouraging me to start pursuing a Ph.D. degree when I joined VTT.

It is also a great pleasure to thank all my colleagues at VTT and elsewhere, who have somehow been involved in the work resulting this dissertation. This includes a long list of people, who have co-authored the original papers included in this thesis or who have participated the related projects. I give special thanks to Mr. Markus Luoto, Ms. Helena Rivas, Mr. Jordi Ortíz, and Mr. Mikko Myllyniemi for their hard work in developing the prototypes and testbeds as well as Mr. Esa Piri and Mr. Mikko Uitto for their assistance with the OMNeT++ simulations. I am also grateful to Dr. Pertti Raatikainen, Mr. Janne Vehkaperä, and Mr. Juha Zidbeck for their valuable comments when finalizing this thesis, and to Dr. Ian Marsh for his help with the language as well as the fruitful discussions on the topic. To everyone else, be assured that your contribution has not been overlooked even though I am not listing all your names here. And in case I missed someone, I am deeply sorry for that.

I thank VTT and its staff for providing the facilities for running all the interesting projects. The availability of the Converging Networks Laboratory and the OPNET simulation tool were crucial for this work. I also thank the people of the Communication Networks knowledge centre for offering me a pleasant working environment.

Finally, I would like to thank my family and friends for their support in this project. I am most indebted to my parents for the support and encouragement they have always given me. And to everyone else in my life: thank you for being there for me. 


\section{Abbreviations}

2G/3G/4G Second, Third, and Fourth Generation Cellular Technology

3GPP Third Generation Partnership Project

$\mathrm{ABC}$

Always Best Connected

$\mathrm{AC}$

Access Category

ADTE

Adaptation Decision-Taking Engine

AN

Access Network

ANDSF

Access Network Discovery and Selection Function

AQPE

Application QoS Policy Engine

AP

Access Point

BL

Base Layer

BS

Base Station

BSS

Basic Service Set

$\mathrm{CN}$

Core Network / Correspondent Node

CoA Care-of-Address

DCMF Distributed Control and Management Framework

DiffServ Differentiated Services

DQT Dependency ID, Quality ID, and Temporal ID

DSMIP Dual-Stack Mobile IP

EDCA Enhanced Distributed Channel Access

EDCAF Enhanced Distributed Channel Access Function

EL Enhancement Layer

EPC Evolved Packet Core

EPS

Evolved Packet System

FA Foreign Agent

FU Fragmentation Unit

GOP Group of Pictures

HA Home Agent

HIP Host Identity Protocol

HSPA High-Speed Packet Access

IEEE Institute of Electrical and Electronics Engineers

IETF Internet Engineering Task Force 
JSVM Joint Scalable Video Model

L2

L3

LAN

LTE

MAC

MANE

MES

MIIS

MIH

MIHF

MIHU

MIP

MN

NAL

NALU

NES

NIS

OSI

PHB

PMIP

PoA

PSNR

QoS

QoE

RAN

RFC

RQ

RSS

RTP

RTCP

RTSP

RTT

SAE
Link Layer

Network Layer

Local Area Network

Long Term Evolution

Medium Access Control

Media-Aware Network Element

Mobile Expert System

Media Independent Information Service

Media Independent Handover

Media Independent Handover Function

Media Independent Handover User

Mobile IP

Mobile Node

Network Abstraction Layer

Network Abstraction Layer Unit

Network Expert System

Network Information Server

Open Systems Interconnection

Per-Hop Behaviour

Proxy Mobile IP

Network Point of Attachment

Peak-Signal-to-Noise-Ratio

Quality of Service

Quality of Experience

Radio Access Network

Request for Comments

Research Question

Received Signal Strength

Real-time Transport Protocol

RTP Control Protocol

Real Time Streaming Protocol

Round-Trip Time

Systems Architecture Evolution 


$\begin{array}{ll}\text { SDP } & \text { Session Description Protocol } \\ \text { SNMP } & \text { Simple Network Management Protocol } \\ \text { SOM } & \text { Self-Organizing Map } \\ \text { SVC } & \text { Scalable Video Coding } \\ \text { TCP } & \text { Transmission Control Protocol } \\ \text { TFRC } & \text { TCP-Friendly Rate Control } \\ \text { TRG } & \text { Triggering Engine } \\ \text { UDP } & \text { User Datagram Protocol } \\ \text { UMTS } & \text { Universal Mobile Telecommunication System } \\ \text { VSS } & \text { Video Streaming Server } \\ \text { WiMAX } & \text { Wireless Interoperability for Microwave Access } \\ \text { WLAN } & \text { Wireless Local Area Network }\end{array}$




\section{List of original papers}

This thesis is based on the following papers, which are referred to in the text by their

Roman numerals (I-V):

I Sutinen T* \& Huusko J (2010) MAC layer QoS architecture for optimized and fair transmission of scalable video. In IEEE Consumer Communications and Networking Conference (CCNC '10), Las Vegas, Nevada, USA: 421-425.

II Sutinen T*, Vehkaperä J, Piri E \& Uitto M (2012) Towards ubiquitous video services through scalable video coding and cross-layer optimization. EURASIP Journal on Wireless Communications and Networking 2012:25.

III Luoto M \& Sutinen T* (2008) Cross-layer enhanced mobility management in heterogeneous networks. In IEEE International Conference on Communications (ICC '08), Beijing, China: 2277-2281.

IV Sutinen T* \& Rivas H (2011) Multi-Interface extension to a scalable video streaming architecture. Journal of Communications 6(9): 700-710.

V Ojanperä T, Luoto M, Ortiz J \& Myllyniemi M (2012) Integrating adaptive video streaming service with multi-access network management. Mobile Networks and Applications 17(4): $492-505$.

*Ojanperä née Sutinen 


\section{Contents}

Abstract

Tiivistelmä

$\begin{array}{ll}\text { Preface } & 7\end{array}$

Abbreviations 9

$\begin{array}{lr}\text { List of original papers } & 13\end{array}$

$\begin{array}{ll}\text { Contents } & 15\end{array}$

1 Introduction to the thesis 17

1.1 Demand for advanced support mechanisms in video streaming . . . . . . 18

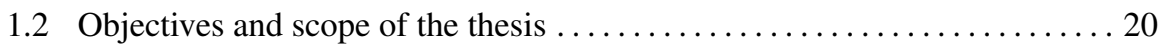

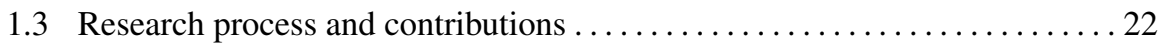

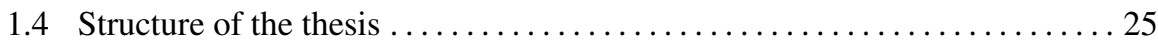

2 Background 27

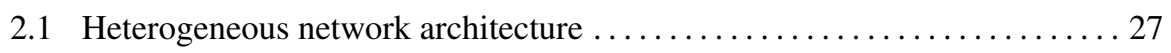

2.1.1 Wireless access technologies....................... 27

2.1.2 Integrating access networks.......................... 34

2.1.3 Heterogeneous network management ................. 40

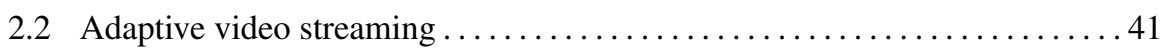

2.2.1 Video streaming system architecture $\ldots \ldots \ldots \ldots \ldots \ldots \ldots \ldots 4$

2.2.2 Impact of scalable video coding . . . . . . . . . . . . . . . . 44

2.2.3 Application layer QoS management .................. 48

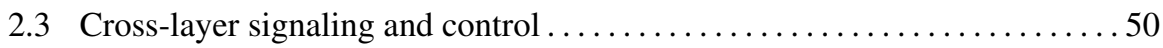

2.3.1 The purpose of cross-layer information in the thesis ..........50

2.3.2 Cross-layer frameworks ........................ 52

2.3.3 Controller coordination ........................... 56

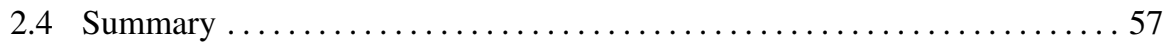

\section{Contributions of the thesis: cross-layer optimization for video}

streaming $\quad 59$

3.1 Cross-layer architecture for video streaming . . . . . . . . . . . . . . 59

3.2 Cross-layer enhancements to video adaptation $\ldots \ldots \ldots \ldots \ldots \ldots \ldots \ldots$

3.2.1 MAC layer scheduling and prioritization ................64

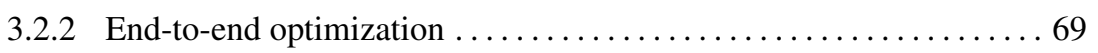


3.2.3 Discussion ................................... 71

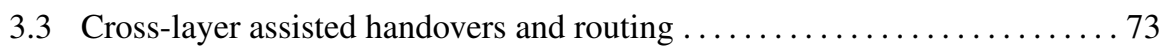

3.3.1 Mobile IP handover performance enhancement ............ 73

3.3.2 Multipath video streaming. ........................ 78

3.3 .3 Discussion .................................. 82

3.4 Coordinated video adaptation and mobility management $\ldots \ldots \ldots \ldots \ldots 83$

3.4.1 Overview of the proposed solution $\ldots \ldots \ldots \ldots \ldots \ldots \ldots \ldots \ldots 4$

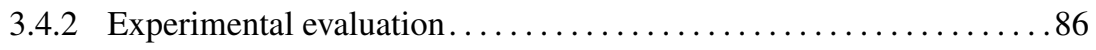

3.4 .3 Discussion ................................ 90

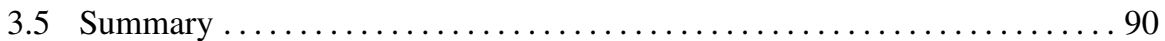

4 Conclusions and future work $\quad 93$

$\begin{array}{lr}\text { References } & 97\end{array}$

$\begin{array}{lr}\text { Original papers } & 105\end{array}$ 


\section{Introduction to the thesis}

Wireless and mobile access to the Internet has become commonplace over the past few years. This development has been enabled by the proliferation of portable handsets such as smartphones and tablets with multi-radio networking capabilities, high processing power, and popular network applications. In addition, there is a variety of access networks and technologies available to a user especially in urban areas, including 2G/3G/4G mobile networks, Wireless Local Area Networks (WLAN), and Wireless Interoperability for Microwave Access (WiMAX). Thanks to the various inter-working solutions proposed for IP-based access networks even from the early 1990s onwards (e.g. [1-3]), these technologies can be combined into a heterogeneous network architecture to enable a ubiquitous network service.

Specific terms have been established in the context of heterogeneous networks, including multi-access, which is used to refer to the simultaneous availability of multiple access networks to a user in the same location; vertical handover, meaning the process of transferring the user's communication sessions between two network points of attachment (PoA) of different technologies; and multi-homing, referring to a situation where a terminal device is capable of communicating over multiple networks, simultaneously.

The access diversity in a heterogeneous multi-access network has obvious advantages, captured by the always best connected (ABC) concept [4]. It refers to a scenario where a user whose terminal device supports multiple access technologies can always use the best available access by dynamically routing the traffic through the most suitable network(s) at hand. Here, the definition of best may be based on various criteria, such as the user's personal preferences, device capabilities, application requirements, operator or corporate policies as well as the characteristics of the available networks. However, allowing a user terminal to roam freely among networks based on differing technologies and managed by different parties introduces a great deal of complexity to access selection, handover execution, and Quality of Service (QoS) management. The individual access technologies have differing functional characteristics and the status of the networks varies; thus the choice for an access network has a direct impact on the performance of the user's networked applications. Especially vulnerable are applications 
offering QoS-sensitive video streaming and thus requiring certain throughput, delay, and loss characteristics from the network connection.

This dissertation studies the problem of supporting video streaming services efficiently in heterogeneous networks with the main focus on wireless access. This introductory chapter motivates the topic as well as introducing the research field in more detail together with an overview of the contributions of this thesis.

\subsection{Demand for advanced support mechanisms in video streaming}

Internet-based mobile video is gaining popularity among users [5], and there is an increasing demand for real-time or near real-time video streaming. Video streaming [6] is, however, particularly sensitive to network QoS. Successful video stream delivery requires certain throughput, which may be in the order of few hundreds of kbps to tens of Mbps depending on the video content and coding [7, 8]. Additionally, small delay and delay variation (jitter) as well as packet loss need to be considered. Network congestion is thus a major problem for video streaming that needs to be alleviated as it causes throughput degradation through random packet drops, eventually having a negative impact on the user-perceived service quality, also known as the Quality of Experience (QoE). On the other hand, due to the constant increase of traffic loads in wireless networks [5], network operators and service providers are looking for cost-effective solutions for managing the traffic demands efficiently and maintaining sufficient QoE for their customers. For instance, this has recently reactivated the discussions on enabling the co-use of 3 GPP mobile data networks with WLANs in an off-loading scenario to integrate WLANs into the mobile broadband strategy of network equipment manufacturers.

In heterogeneous wireless networks, a video streaming session may experience congestion, for example, due to inappropriate access technology selection or competing traffic in the network or the wireless link. Thus, the video service QoS requirements need to be taken into account in the access selection and handover decision-making. Moreover, the mobility solution should be able to harness the available transmission resources efficiently by allowing concurrent use of multiple networks, if feasible, in order to maximize the video streaming QoE. In addition, video streaming services that do not implement required mechanisms to react to congestion will continue contributing 
to it and thus waste precious network resources, for instance, if the receiver is not receiving all the video data needed for decoding due to congestion-induced packet drops and delays, the partial or delayed information successfully received may be useless and thus be dropped in the receiver. This is a problem especially for User Datagram Protocol (UDP) -based video streaming where the transport layer does not implement any congestion control. Therefore, video adaptation needs to be supported by the application in order to adjust the video stream to the available network capacity as far as possible. The support for adaptation also relaxes the QoS requirements of the video streaming; thus, potentially increasing the number of access options in a heterogeneous multi-access network.

Nevertheless, the problem with existing adaptive video streaming and mobility management systems is the lack of sufficient control over video streaming and terminal mobility so that they could be utilized for optimizing video streaming in heterogeneous wireless networks. For instance, the current mobility protocols such as the Mobile IP (MIP) [2, 9-13] base their handover decisions on limited information that does not reflect the actual transmission conditions in the networks nor do they take into account any application-specific requirements in decision-making. The protocols also hide mobility from the upper layers making it impossible for QoS-sensitive applications like video streaming to adapt in time in the event of vertical handovers. Current application layer video adaptation solutions, including, for example, those based on the TCP Friendly Rate Control (TFRC) [14-17], also have difficulties in operating over network paths with wireless links due to their reliance on limited end-to-end feedback signaling. Thus, the current solutions and protocols for mobile video streaming can lead to increased congestion and unnecessary data transmissions.

Cross-layer design [18] provides one solution for realizing the advanced signaling and control for the video adaptation and mobility management mechanisms. This dissertation aims to show that cross-layer aided, QoS-aware video adaptation and mobility control improve video streaming performance significantly in heterogeneous wireless networks. For this purpose, the thesis presents novel mechanisms for optimizing video stream delivery in a multi-access environment considering the prevailing channel characteristics of the different access networks, user/operator policies, as well as the video application's capabilities and QoS requirements. 


\subsection{Objectives and scope of the thesis}

The problem of supporting video streaming services in heterogeneous wireless networks is addressed in the context of three research questions (RQ) in the thesis. They are discussed in this section together with the scope of the thesis.

The RQs to be answered by the thesis are: RQ1: How to design and implement a cross-layer signaling and controlling system in order to enable efficient and timely mobility and video adaptation decision-making in heterogeneous wireless networks? The elaboration of this problem includes consideration of the system architecture and introducing new entities required for the cross-layer signaling and controlling functions. RQ2: What kind of cross-layer optimized functions can be used to support video transmission in heterogeneous wireless networks so that it utilizes the available network resources efficiently and fairly? Heterogeneous networks with simultaneous availability of multiple access networks and technologies offers great potential for harnessing the available transmission capacity for video streaming purposes. This can be enabled by allowing the client terminal to connect to the video streaming service dynamically through the best possible access at all times and locations. Nevertheless, for efficient and fair use of network resources, it should be ensured that the video streaming does not reserve transmission resources beyond its fair share. This thesis addresses the problem by studying video stream adaptation and routing as means for video streaming services to make the best use of the available network capacity in a multi-access environment while at the same time maintaining fairness to other network traffic. And finally, RQ3: How to ensure sufficient coordination between the introduced cross-layer functions for stability and performance? This problem is addressed in the context of video rate adaptation and congestion-triggered handovers that both attempt to alleviate the same network problem, namely congestion.

The dissertation considers a video streaming system where the client device supports one or more wireless network interfaces and technologies. In addition, continuous connectivity is assumed, that is, that there is always at least one access network available to the terminal. The video server, on the other hand, is located in the wired network. Throughout the dissertation, it is assumed that the bottleneck link between the video server and client is the directly connected wireless link of the client. That is to say, multi-hop wireless scenarios are not considered as such. Nevertheless, the application layer video adaptation solutions using end-to-end feedback on the video streaming performance studied in this thesis are able to adjust the video bitrate to available network 
capacity in the end-to-end scope. However, the other proposed optimization mechanisms focus on alleviating transmission problems primarily in the scope of the wireless link or the access network. Thus, for the other solutions apart from the ones supporting end-to-end optimization, extensions to the proposed approaches would be required to handle video stream optimization in multi-hop wireless networks, present, for instance, in many WLAN offerings running on vehicles nowadays.

The thesis is concerned with video streaming optimization at the application and network layers as well as the medium access control (MAC) sublayer of the data link layer. Yet, it is acknowledged that further optimization may be achieved by adopting additional mechanisms on other protocol layers, including, for example, different error resilience mechanisms in the transport layer, robust header compression between the network and data link layers as well as optimizing video packet transmission at the physical layer $[19,20]$. These are, however, not considered in this thesis. In addition, the work focuses strictly on video transport and rate shaping type of adaptation, and consequently video encoding and decoding details are beyond the scope of this thesis.

Regarding video streaming performance, the dissertation considers different metrics. First, $Q o S$ refers to the performance of the network in transferring the video data between the source and destination. QoS can be measured objectively with metrics such as video stream throughput, video packet delay, and video packet loss. Second, $Q o E$ is a measure that registers the user's perception on the performance of the video streaming service. Here, QoS is only one factor in how good or bad the received video is finally graded by the user. The video encoding and the parameter values used in this as well as the performance of the decoder application also have an impact on the QoE and affect the video stream requirements regarding transport and display [7]. Moreover, an encoded video bitstream contains frames of differing importance to the receiver; thus, the loss of a video packet may have a different impact on the QoE depending on its type. However, this thesis does not go deeply into the problems of QoE estimation due to its highly subjective nature and complexity. That is, any subjective evaluation of video QoE (e.g. Mean Opinion Score, MOS) is beyond the scope of the thesis, and only objective metrics are used when available. Regarding the encoding parameters, the thesis uses pre-encoded videos; thus, the solutions only assume that the basic quality of the lowest supported adaptation level is acceptable to the user. Last, fairness is considered in the thesis in terms of ensuring that the video session does not consume limited network resources beyond its allocated or fair share. That is, the video streaming must not violate the network resource allocation defined by the operator and enforced, for example, with 
MAC layer scheduling algorithms. In the case of UDP-based video streaming, this also means that the video streaming should incorporate congestion control mechanisms to coexist with TCP sessions. In the thesis, fairness is mainly evaluated by measuring the QoS also experienced by other traffic in the network besides video.

\subsection{Research process and contributions}

The thesis proposes novel solutions for improving video streaming performance in heterogeneous wireless networks. A key enabler for the solutions is cross-layer signaling which facilitates more informed decision-making in different system entities that contribute to the performance optimization of the video streaming. First, to support the required cross-layer information collection and signaling, this thesis proposes new components and protocols for the video streaming system architecture. Then, the thesis proposes cross-layer assisted algorithms for video rate adaptation, networklayer handovers, and multipath video streaming. Each of these provides a solution for improving the QoS and QoE of video streaming under limited network capacity. Moreover, the video rate adaptation is designed so that it maintains fairness towards other network traffic. Finally, to achieve a more complete solution in terms of performance optimization and management, this thesis considers supporting the cross-layer enhanced video rate adaptation and mobility management functions simultaneously in the video streaming system. For this purpose, this thesis proposes a solution for coordinated management of the optimization functions operating on distinct protocol layers for efficiency and improved performance.

The research for this thesis was mainly conducted in large national and international research projects, which provided the framework and processes for the work all the way from the initial requirements analysis to prototyping and evaluation. The quality of the results was assured by regular reviews by the financier among others. In addition, the prototypes were showcased in various events to collect feedback from experts outside the projects.

The experimental evaluation of the solutions proposed in this thesis was conducted using both network simulations and real prototype implementations. The reasoning behind this two-fold approach originates mainly from the fact that implementing and experimenting lower protocol layer functionalities (e.g. MAC) is faster and more reliable in a simulator than with real network equipment. Two advanced network simulators were used in the experimentation, namely OPNET and OMNeT++. Nevertheless, prototypes 
were used to demonstrate the feasibility and operation of the higher protocol layer functionalities in laboratory testbeds.

This dissertation is based on five peer-reviewed original papers that in the rest of the thesis are referred to as Paper I, II, III, IV, and V. In the following, the topics covered in the original papers are introduced together with the contributions of the author.

Paper I: The paper introduces a MAC layer QoS architecture for optimizing scalable video delivery across wireless links. The architecture implements prioritized queuing and scheduling of video packets in order to produce differentiated treatment for video data within the video traffic QoS category. The main benefit obtained is that at least the most important video packets get through a resource-constrained wireless link to support service continuity. Moreover, the QoE is improved whenever the network conditions allow. The MAC layer QoS architecture is designed so that the optimized video transmission does not hamper the QoS allocated to other types of traffic. The paper presents an experimental evaluation on the concept through a simulation study, where the IEEE 802.11e Enhanced Distributed Channel Access (EDCA) QoS architecture is extended with the proposed video traffic queuing and scheduling features. In addition, the system utilizes the Scalable Video Coding (SVC) technology for producing a stream of video packets with an impact that is different to the resulting video presentation quality. The author participated in the design of the proposed MAC layer QoS architecture and was responsible for the implementation of the simulation model as well as the planning and carrying out the experiments, and analysing the results. The author was also the main contributor to writing the paper with input from the co-author Jyrki Huusko.

Paper II: The paper presents a system architecture supporting cross-layer adaptation of video streams. The architecture implements end-to-end application layer video adaptation based on the TFRC algorithm and supports MAC layer video packet differentiation using the MAC layer QoS architecture proposed in Paper I. The adaptation is controlled with a cross-layer signaling and controlling architecture proposed in the paper. The application layer adaptation was introduced in order to reduce unnecessary packet transmissions in the wired core network whenever the wireless link is unable to support the whole video stream. The MAC layer video packet differentiation, on the other hand, enhances video streaming performance QoE-wise as it is able to react to wireless link fluctuations faster than the application layer adaptation. The experimentation was conducted with network simulations and utilizing the SVC technology. The author of 
this thesis took part in the design of the architectures specified for the adaptive video streaming system as well as the cross-layer signaling and control. Moreover, the author contributed to the implementation of the simulation model. The author also participated in the planning and executing of the experiments, analysing the results, and was the main author of the paper with input from the co-authors: Janne Vehkaperä, Esa Piri, and Mikko Uitto.

Paper III: The paper presents the design and prototype implementation of a network layer mobility management solution that utilizes cross-layer information to support more informed handover decision-making in heterogeneous multi-access networks. The paper introduces a cross-layer signaling and controlling architecture that supports the gathering of extensive context information from the mobile terminal as well as from the network for handover management purposes. In the paper, a commercial MIPv4 implementation was integrated with the cross-layer architecture and the cross-layer information was used in implementing advanced control over MIP's handover decision-making. The experimental evaluation of the cross-layer enhanced MIP handover mechanism was conducted in a laboratory network environment using real equipment. The author of this thesis participated in the design of the cross-layer signaling architecture and the MIP prototype as well as the cross-layer enhanced MIP handover decision-making algorithms. The author also took part in the planning of the experiments as well as in the analysis of the results. The author acted as a supervisor for the master's thesis of co-author Markus Luoto, who implemented the prototype and conducted the experiments as a part of his thesis [21]. The author had a significant role in the writing of the paper with input to the leading author Markus Luoto.

Paper IV: The paper presents an architecture for adaptive transmission of scalable video streams in heterogeneous networks and its required extensions to support multipath or multi-interface video streaming. The objective was to enhance the QoE by enabling video stream reception concurrently through multiple access networks. Moreover, to cope with the dynamic behaviour of heterogeneous multi-access networks, the paper proposes interfacing the multipath video streaming system with a cross-layer signaling architecture for dynamic mapping of SVC layers to available access networks for transport. The paper presents a prototype implementation of the proposed solution together with experimental evaluation conducted in a laboratory environment. The author participated in the design of the multipath SVC streaming system and specified 
its integration with the cross-layer signaling architecture. The author also contributed to the design of the prototype as well as to the planning of the experiments and analysis of the results. In addition, the author was the main author of the paper with input from the co-author Helena Rivas.

Paper V: The paper combines video adaptation and network-layer mobility management to optimize video streaming in heterogeneous multi-access networks. The solution extends the common cross-layer signaling and controlling architecture introduced in Paper III to support the video streaming components presented in Paper IV (excluding the multipath features). The paper applies cognitive algorithms in detecting problems in the network and implements a control algorithm for triggering video stream adaptation and handovers accordingly in a coordinated manner in the mobile terminal. In addition to presenting a complete system architecture realizing the proposed functionality, the paper introduces a prototype including various open source components for adaptive SVC streaming and MIPv6-based mobility management. The paper presents an experimental evaluation of the prototype conducted in a laboratory environment. The author of this thesis participated in the design of the system and the implementation of the prototype. In addition, the author took part in the planning and execution of the experiments as well as in analysing the results. The author was also the main author of the paper with input from the co-authors: Markus Luoto, Jordi Ortíz Murillo, and Mikko Myllyniemi.

\subsection{Structure of the thesis}

The remainder of this thesis is organized as follows. Chapter 2 presents the theoretical background with an overview of related work. The chapter forms the basis for the discussion of the solutions proposed in this thesis by introducing the building blocks of the considered network and service architectures and motivating the need for cross-layer control in optimizing video streaming in heterogeneous wireless networks. Chapter 2 also presents the key technologies and standards relevant to this thesis, including different wireless access network technologies, IP mobility management protocols and scalable video coding as well as existing cross-layer signaling frameworks. Chapter 3 introduces the solutions and results presented in the original papers for cross-layer optimized video streaming in heterogeneous wireless networks. Finally, Chapter 4 concludes the thesis and discusses directions for future work. 


\section{Background}

In this chapter, the theoretical background for the dissertation is presented with references to the literature. This chapter forms the basis for the discussion on the solutions proposed in Chapter 3 for enhancing video streaming performance in heterogeneous wireless networks. The current chapter begins with a discussion on the concept and composition of a heterogeneous wireless network architecture in Section 2.1. Then, Section 2.2 provides an introduction to adaptive video streaming systems with a specific interest on scalable video transmission and adaptation. The concept of cross-layer optimization is introduced and its impact on the current communication architecture is discussed in Section 2.3. Finally, Section 2.4 summarizes the topics discussed in this chapter.

\subsection{Heterogeneous network architecture}

Heterogeneous networks consist of multiple access technologies and networks with overlapping coverages and are accessed with multi-mode user terminals. Heterogeneous networks essentially enable the $\mathrm{ABC}$ scenario, where a user is able to utilize the advantages of the different networks and technologies by dynamically routing the traffic through the best available access network(s) at all times. This section discusses the principles of wireless access to IP networks and introduces the main IP-based wireless access network technologies in use today. In addition, the integration of the individual technologies into a heterogeneous network architecture is considered.

\subsubsection{Wireless access technologies}

A wireless access network (AN) connects a user terminal device (also referred to as a station, a host or a node) to the wired network infrastructure through a wireless PoA, called an access point (AP) or a base station (BS) depending on technology. The communication between the user terminal and the PoA takes place over the air using radio signals. Thus, wireless networks have differing characteristics from their wired counterparts, such as unreliability, typically lower bandwidth, broadcast type-of communication, and support for mobility [12]. These characteristics have an impact on how wireless networks are used as well as on the operation of many communication 
protocols, originally designed with only fixed networks in mind. This section provides an introduction to IP-based wireless network technologies relevant for mobile video services as well as their data transmission, QoS, and mobility capabilities.

\section{Wireless local area network (WLAN)}

WLANs provide low-cost wireless network access to small geographical areas, and they have become a popular Internet access method in offices, airports, hotels, coffee shops as well as private homes. The affordability of the technology originates from the usage of unlicensed frequency bands and inexpensive network equipment. In addition, most of today's consumer equipment (e.g. smartphones and laptops) have support for WLAN through a network interface card supporting one or several of the IEEE 802.11 physical layer standards [22].

The IEEE 802.11 WLAN architecture [12, 22] supports two modes of operation: ad hoc, in which wireless stations in a given area communicate with one another on a peer-to-peer basis, and infrastructure, in which the wireless stations are connected to a backbone network and onwards to different networked services via APs. This dissertation focuses on considering the latter, also depicted in Figure 1.

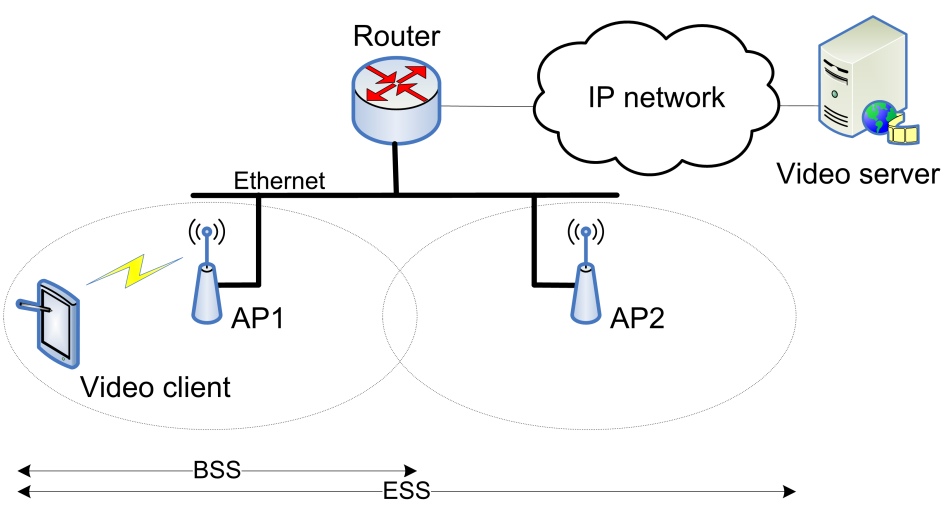

Fig 1. The WLAN architecture - Infrastructure mode.

In the infrastructure topology, the AP provides access to the backbone network and services (e.g. the video server in Figure 1) via the wireless medium for the stations (i.e. the video client in Figure 1). The transmission capacity of an AP is shared among the wireless stations connected to it. The set of stations controlled by an AP forms a basic 
service set (BSS), whereas several BSSs interconnected with a distribution system (i.e. a IEEE 802.3 LAN in Figure 1) form an extended service set (ESS). The ESS mediates traffic between the BSSs belonging to it. The IEEE 802.11 standard also defines a logical entity called a portal (i.e. the router in Figure 1), which is the logical point of the ESS, where the IEEE 802.11 network interfaces with some other non-802.11 packet network. Finally, mobility of the stations is supported in three modes: no transition, a BSS transition, and an ESS transition. Only in the last case may the IP address of the wireless station change.

Nowadays, the IEEE 802.11 standards family includes one MAC and multiple physical (PHY) layer implementations for the wireless link with distinct characteristics $[12,22]$. From the currently used PHY standards, the data rates supported by IEEE $802.11 \mathrm{~b}$ in the $2.4 \mathrm{GHz}$ band are between 1 and $11 \mathrm{Mbps}$; IEEE 802.11a supports data rates up to $54 \mathrm{Mbps}$ in the $5 \mathrm{GHz}$ band; IEEE 802.11g supports data rates up to $54 \mathrm{Mbps}$ in the $2.4 \mathrm{GHz}$ band; and IEEE $802.11 \mathrm{n}$, as the latest amendment, provides data rates of at least $100 \mathrm{Mbps}$ in both the 2.4 and $5 \mathrm{GHz}$ bands using the MIMO technology. All the PHY standards share the same MAC layer implementing the carrier sense multiple access with collision avoidance (CSMA/CA) protocol as the basic contention-based access mechanism. More details on the PHY and MAC functions can be found from $[12,22]$. WLANs support one-way transmission delays (i.e. latencies) of around $10 \mathrm{~ms}$.

The IEEE 802.11 standards' family also includes several supplemental standards to enhance the QoS, security, mobility, etc. in WLANs. The QoS-related amendments are the IEEE 802.11e, incorporated into the current version of the IEEE 802.11 standard [22], and the recently published IEEE 802.11aa [23]. This thesis considers the IEEE 802.11e EDCA mechanism, which implements QoS for WLAN links by dividing packets coming from higher layers (e.g. IP) into four queues representing the different EDCA access categories (AC), that is, AC_VO for voice, AC_VI for video, AC_BE for best effort and AC_BK for background traffic, depending on their priority. EDCA implements priority- and contention-based channel access for the ACs through the enhanced distributed channel access function (EDCAF). The priority-based access for an $\mathrm{AC}$ is achieved through varying the EDCAF parameters, in a per-AC basis. Figure 2 illustrates the reference implementation model of EDCA.

Whereas EDCA focuses on implementing priority-based access for different traffic types, the 802.11aa specifies mechanisms to better support video and audio streaming in WLANs, while maintaining co-existence with other types of traffic. The amendment defines intra-AC priorities and additional queues for audio and video packets as well 


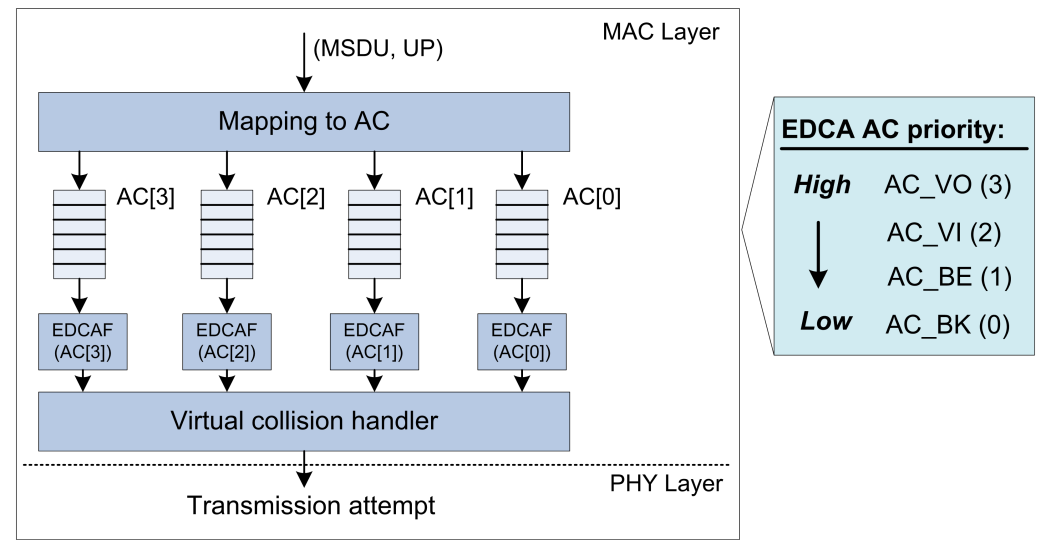

Fig 2. EDCA reference implementation model for transmitting IEEE 802.1D user priority (UP) marked MAC service data units (MSDU) across a WLAN link.

as the means for signaling drop eligibility of a packet in the MAC header to enable graceful degradation of audio video streams under insufficient channel capacity.

\section{Universal mobile telecommunication system (UMTS) and evolution}

The 3GPP technologies [3, 24-26], providing users with mobile telephony and data services, are the dominant mobile network technologies in use today. The evolution path of the $3 \mathrm{GPP}$ systems has gone through several stages starting from the $2 \mathrm{G}$ technologies (i.e. GSM/GPRS/EDGE) to 3G (i.e. WCDMA/HSPA/HSPA+) and onwards to 4G (i.e. LTE/LTE-Advanced). Coexistence and interworking is supported between the different technologies, as illustrated in Figure 3. However, due to the limited data rates of the $2 \mathrm{G}$ technologies [24], as regards video transmission, this section focuses on discussing the $3 \mathrm{G}$ and $4 \mathrm{G}$ technologies in terms of UMTS and especially its HSPA enhancement as well as LTE. Moreover, due to the focus on data services, the 3GPP voice communication aspects are excluded from the discussion. More details on the 3GPP family of technologies and their evolution can be found from [24-26].

The network architecture of the 3GPP systems consists of the core network $(\mathrm{CN})$, different radio access networks (RAN), and the IP multimedia subsystem (IMS) [3, 26]. Figure 3, depicts a generalized 3GPP network architecture, highlighting the components in the data path towards an external packet data network (e.g. Internet) as well as those handling mobility management. The 3GPP RANs include the GSM/EDGE radio access network (GERAN), the UMTS terrestrial radio access network (UTRAN) as well 


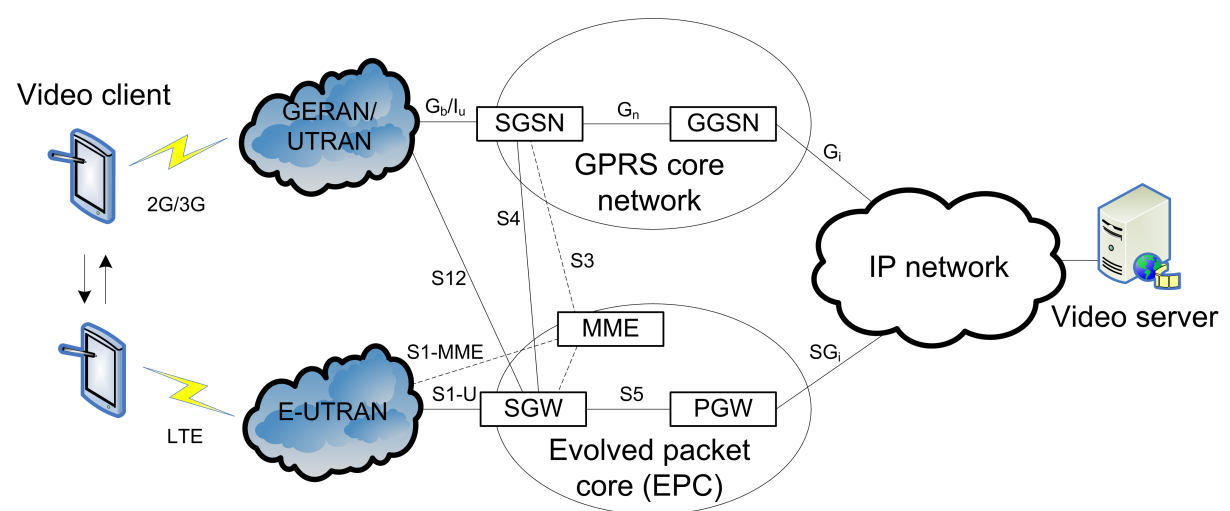

Fig 3. Generalized network architecture (non-roaming) for 3GPP (2G/3G/LTE) access.

as the evolved UTRAN (E-UTRAN). Mobility is supported within and between the RANs. The GERAN and UTRAN share the same CN, namely the GPRS core network, containing the two main entities for packet data transmission: the serving GPRS support node (SGSN) and the gateway GPRS support node (GGSN). The former handles the routing of packets between the external packet data network (PDN) and a mobile station located in the routing area of the SGSN. The latter acts as a gateway between the GPRS $\mathrm{CN}$ and other PDNs. The GSNs are interconnected via the GPRS Tunnelling Protocol (GTP). The E-UTRAN connects to a new $\mathrm{CN}$ architecture, called the evolved packet core $(E P C)$. Also, the EPC includes gateways for handling the user plane traffic, namely the serving gateway (S-GW) and the packet data network gateway (P-GW), which may be optionally implemented as a single system architecture evolution gateway (SAE GW). The gateway interconnection may be realized either with GTP or Proxy Mobile IP (PMIP). The mobility management entity (MME) is the main control entity in EPC that handles the mobility and bearers for mobile stations connected through E-UTRAN. A more detailed description of the 3GPP RAN and CN architectures and their components can be found from [24-26]. Furthermore, Figure 3 also shows some of the interfaces defined in the $3 \mathrm{GPP}$ inter-working system architecture to allow optimized interworking between E-UTRAN, UTRAN, and GERAN. More details on the 3GPP inter-working system architecture can be found from [26].

In 3GPP, the theoretical maximum data rates have evolved from $358 \mathrm{Kbps}-2 \mathrm{Mbps}$ of WCDMA (UMTS), to $14 \mathrm{Mbps}$ of HSPA, to 21-168 Mbps of HSPA+ as well as to 150-300 Mbps of LTE and 1 Gbps of LTE-A [26]. The round-trip packet transmission 
delay (RTT) in UMTS is around 150-200 ms [25], whereas in LTE RTTs below $15 \mathrm{~ms}$ can be achieved [26]. In HSPA/HSPA+, the RTT is 50-100 ms [25].

$3 \mathrm{GPP}$ has also defined mechanisms for supporting QoS in its mobile data networks [24-27]. For UMTS, four service classes are supported, depending on the service's real-time requirements, namely conversational, streaming, interactive, and background $[24,25]$. In E-UTRAN/EPC, the concept of an evolved packet system (EPS) bearer $[26,27]$ is introduced for enforcing differentiated treatment for data flows. An EPS bearer maps to a flow or a set of flows established between the P-GW and the user terminal, and is associated with the following QoS parameters: the QoS class identifier (QCI), allocation and retention priority (ARP), maximum bitrate (MBR), guaranteed bitrate (GBR), and aggregate maximum bitrate (AMBR), when multiple IP flows are mapped to the same bearer. The QCI breaks down to nine pre-defined levels of priority, delay, and loss rate for either GBR and non-GBR type of bearers. The QCIs may be mapped to different types of applications (e.g. VoIP, video call, streaming, etc.) in order to realize their required QoS. A bearer may also be either a default or dedicated bearer. A default bearer always exists for each terminal IP address and provides basic connectivity with a subscription-dependent level of QoS, which nevertheless is always a non-GBR service. In addition to the default bearer, a user terminal may request one or several dedicated bearers to obtain differentiated treatment for its data flows.

\section{Other access technologies}

Despite focusing on WLAN and 3GPP technologies in this section, there are also other IP-based access technologies relevant for the discussion of heterogeneous network environments. One is the well-known WiMAX standard [28, 29] for metropolitan area networks (MAN). WiMAX is based on the IEEE 802.16 standards family, and is promoted by the non-profit, industry-led WiMAX Forum. Two versions of the standard are supported today, namely the fixed WiMAX and mobile WiMAX, both included in the current version of the IEEE 802.16 standard [30]. The mobile WiMAX, which is comparable to the WLAN and 3GPP systems for its mobility support, provides shared data rates of $35 \mathrm{Mbps}$ per $10 \mathrm{MHz}$ channel with a user plane latency of less than $20 \mathrm{~ms}$. There is also a new amendment to the WiMAX standard, that is, the IEEE 802.16m [31], defining an advanced air interface with data rates greater than $100 \mathrm{Mbps}$ per sector for a $20 \mathrm{MHz}$ channel for mobile use with a user plane latency of less than $5 \mathrm{~ms}$. The IEEE $802.16 \mathrm{~m}$ air interface has been designated as 'IMT-Advanced' (i.e. an official 4G 
technology) by the International Telecommunication Union's Radiocommunication Sector (ITU-R) together with LTE-A. The MAC layer of WiMAX supports QoS, implemented through five scheduling services, namely unsolicited grant service (UGS), real-time polling service (rtPS), non-real-time polling service ( $n r t P S)$, best-effort service $(B E)$, and extended real-time polling service (ertPS). In addition, although emphasizing wireless access in this dissertation, the wired technologies are considered as an important access alternative in home and office networking scenarios as well as for connecting the application servers to the Internet on the service provider's side. In the case of fixed access, local area networks (LAN) built using the IEEE 802.3 Ethernet technology [32] that supports data rates of $10 \mathrm{Mbps}-10 \mathrm{Gbps}$ and very low latency (1 ms) are mainly used in this thesis.

\section{Comparison and discussion}

Table 1 summarizes some of the characteristics of the AN technologies discussed in this section. Today, the deployment of these technologies has resulted in overlay or multi-access network environments, where multiple networks or technologies are available to a user in the same place or area. Multi-access networks have been appeared because none of the technologies have been found to serve all purposes and needs. In fact, in addition to the differences in data rate, QoS, and mobility capabilities listed in Table 1, the technologies also differ in many other aspects, such as cost (infrastructure and use related), wireless spectrum use and licensing, availability of compatible user terminals, power consumption, deployment of earlier generations, etc., each impacting on the prevalence of the network technology and standard in a given geographical area and purpose of use. On the other hand, the technologies are seen to complement one another coverage-wise as, for instance, WLAN is fitted to provide connectivity in a local scope, whereas WiMAX and 3GPP technologies may be used for covering larger geographical areas (i.e. are suited to MANs or wide-area networks, WAN). The boundaries are, nevertheless, somewhat vague, which creates competition between the technologies (e.g. LTE and WiMAX [29] or LTE and WLAN in the case of femto APs $[25,26])$. Yet, it is generally accepted that there is also a need for multiple access technologies and multi-access environments in the future. In multi-access networks, the available networks also change dynamically as the user moves. Thus, knowing the characteristics of the available networks and any changes in them is an important enabler for optimizing video service delivery in heterogeneous wireless networks. The next 
section continues the discussion by introducing different approaches for integrating individual access networks and technologies into a heterogeneous network architecture.

Table 1. Summary and comparison of IP-based access technologies.

\begin{tabular}{|c|c|c|c|c|}
\hline Technology & Standard & Data rate & QoS & Mobility \\
\hline \multirow[t]{4}{*}{ WLAN } & IEEE 802.11b & 1-11 Mbps & IEEE 802.11e & Low \\
\hline & IEEE 802.11a & 1-54 Mbps & IEEE 802.11e & Low \\
\hline & IEEE $802.11 \mathrm{~g}$ & 1-54 Mbps & IEEE 802.11e & Low \\
\hline & IEEE 802.11n & $>100 \mathrm{Mbps}$ & IEEE 802.11e & Low \\
\hline \multirow[t]{5}{*}{ 3GPP } & WCDMA & $384 \mathrm{Kbps}-2 \mathrm{Mbps}$ & UMTS QoS & High \\
\hline & HSPA & $\leq 14 \mathrm{Mbps}$ & UMTS QoS & High \\
\hline & HSPA+ & $\leq 21-168 \mathrm{Mbps}$ & UMTS QoS & High \\
\hline & LTE & $\leq 150-300 \mathrm{Mbps}$ & EPS bearer & High \\
\hline & LTE-A & $\leq 1 \mathrm{Gbps}$ & EPS bearer & High \\
\hline \multirow[t]{2}{*}{ Mobile WiMAX } & IEEE 802.16 & $\leq 35 \mathrm{Mbps}$ & WiMAX QoS & High \\
\hline & IEEE $802.16 \mathrm{~m}$ & $>100$ Mbps & WiMAX QoS & High \\
\hline LAN & IEEE 802.3 & $10 \mathrm{Mbps}-10 \mathrm{Gbps}$ & No & No \\
\hline
\end{tabular}

\subsubsection{Integrating access networks}

This section discusses the different approaches for integrating access technologies and networks into a heterogeneous network architecture providing mobile users with a seamless and ubiquitous access to networked services. Thus, the discussion includes an overview of different AN integration models and mechanisms, such as terminal mobility, multihoming, and QoS, which are required for supporting the mobile users' ongoing communication sessions and utilizing the available network resources efficiently. The base assumptions made in this section are that the user terminal supports two or more access technologies and that mobility within an AN is handled through the technology-specific mechanisms. A more detailed description of the mobility scopes in heterogeneous network environments is included, for example, in Sutinen [13].

\section{Architectural considerations}

Considering 3GPP and non-3GPP networks (e.g. WLAN and WiMAX), three approaches can be identified for the integration of the ANs into a heterogeneous network architecture: tight coupling, loose coupling, and no coupling. The last two are illustrated in Figure 4 in the context of the 3GPP/WLAN inter-working scenario. 
The tight and loose couplings of non-3GPP access networks to the 3GPP architecture are defined in the 3GPP SAE of 3GPP release $8[3,26]$, which can be seen as the first proper realization of multi-access convergence in 3GPP networks [3]. The tight coupling is supported only in the special case of cdma2000, and other non-3GPP ANs are integrated relying only on loose coupling, that is, without $\mathrm{AN}$-level interfaces. In addition, since the loose coupling method to EPC depends on whether the non-3GPP AN is capable of running 3GPP authentication safely or not, WLANs are, in general, assumed to be un-trusted. Their integration therefore requires a new entity called the evolved packet data gateway (ePDG) in the EPC as shown in Figure 4. The ePDG acts as a gateway to the WLAN providing various networking services, including security, QoS, and mobility management for the user terminal. User terminal mobility between the LTE and WLAN networks may in this case be supported by the Proxy Mobile IPv6 (PMIPv6) implemented in the ePDG or Dual-Stack Mobile IPv6 (DSMIPv6) in a completely user terminal centric solution. In principle, in this mode, the user terminal is able to roam freely between the networks, while receiving the same services as in the 3GPP networks. However, QoS is supported only on the EPC-side, and it is up to the WLAN to implement the packet forwarding treatment for the user's traffic according to the 3GPP QoS parameters [3]. Finally, to support roaming to non-3GPP ANs, 3GPP has defined an optional information service called the access network discovery and selection function (ANDSF) [3].

The non-3GPP inter-working system architecture defined by 3GPP allows efficient utilization and management of multiple access technologies and facilitates, for example, load-balancing [33], off-loading [34], and optimal access to operator managed services offered by the IMS [26]. However, for legacy Internet applications, such as the overthe-top video (OTT) services [35], it may not be feasible always to route all the traffic through the 3GPP CN. In addition, there are broadband network operators who do not have cellular networks, but could still benefit from the ability to integrate their networks into the heterogeneous network architecture. Thus, to avoid limiting oneself to the 3GPP-centric scenario, this thesis considers the third integration approach, that is no-coupling, as the basis for the heterogeneous network architecture.

Focusing on IP-based access networks and technologies in this dissertation, the user terminal mobility support between the ANs can be implemented by using an IP-mobility management mechanism in the no-coupling scenario. The reason why mobility in IP networks requires the introduction of a specific mobility protocol, basically, is the two-fold nature of the IP address: an IP address of a node is used to identify both the 


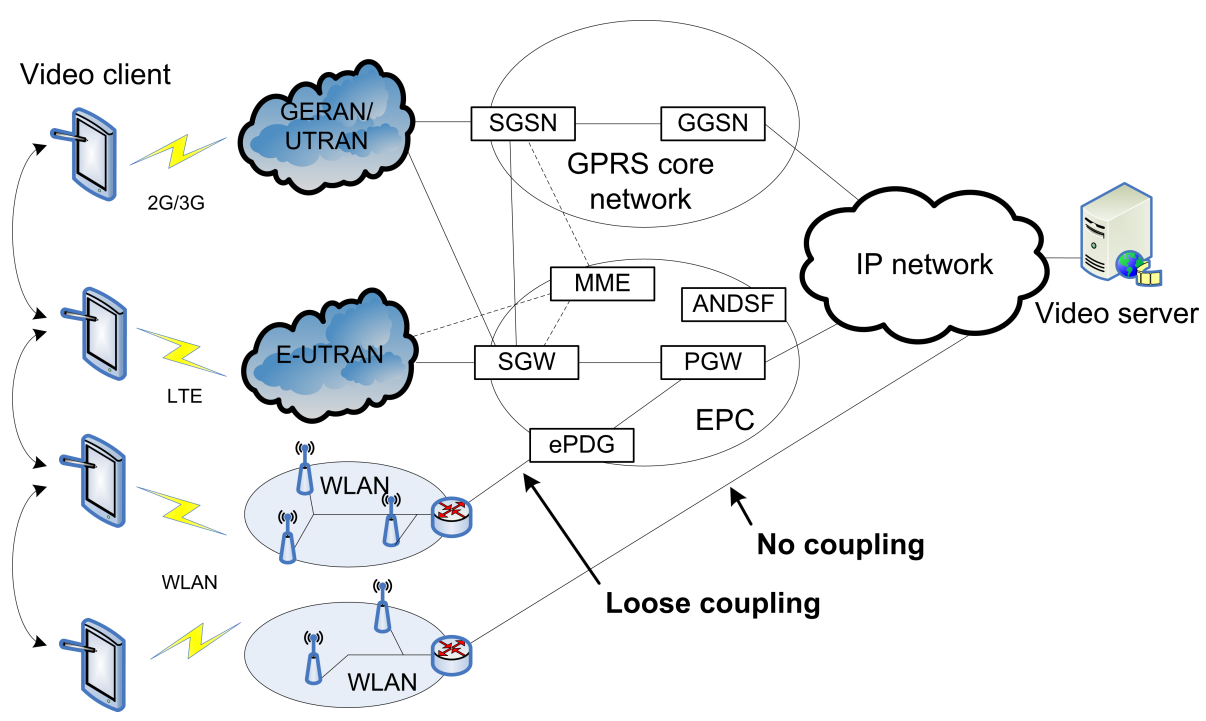

Fig 4. Generalized integrated 3GPP/WLAN architecture.

physical location of the node in the Internet as well as the communication end-points at the application layer (i.e. sockets) [13], thus, the changing of the node's IP address due to mobility breaks any on-going communication sessions. Nevertheless, the impact of this is application dependent [36], that is short-lived sessions such as those involved in web browsing are less likely to suffer from an inter-domain handover than long-lived ones like video streaming.

To ensure continuous connectivity to any Internet services in a heterogeneous network environment, several protocols for IP-mobility management have been specified over the years in the IETF. The mechanisms may be categorized based on the protocol layer they are operating on, and include specific trade-offs discussed, for example in Sutinen [13]. The network-layer mechanisms include the Mobile IPv4 (MIPv4) [9] and MIPv6 [10] as well as their variants (e.g. PMIPv4/v6 and DSMIPv4/v6, Hierarchical MIPv6 as well as Fast Handovers for MIPv6) [3, 26, 37]. The Host Identity Protocol (HIP) $[38,39]$ operates between the network and transport layers by introducing a new protocol layer in the middle. Examples of existing and upcoming transport layer protocols supporting IP mobility include the Stream Control Transmission Protocol (SCTP) [40], Multipath TCP (MPTCP) [41], and Multipath RTP (MPRTP) [42]. Finally, on the application layer, the Session Initiation Protocol (SIP) [43] may be used to handle the mobility. Of the previously mentioned mechanisms, HIP, SCTP, MPTCP, and MPRTP may be used to support multihoming in addition to IP mobility. For MIPv6, 
support for multiple interfaces and paths for the transport of flows can be incorporated through the use of multiple care-of-addresses [44, 45]. For MIPv4, there is currently activity under the IETF to define multipath support for the protocol [46].

The problem of a heterogeneous network enabled through the non-coupling approach is the lack of generic access selection and QoS mechanisms, which are required to support user traffic requirements in such a diverse and dynamic network environment. For AN selection, different network information services, such as the IEEE 802.21 Media Independent Handover (MIH) services framework [47, 48] and the triggering framework [49], have been proposed, and are discussed in more detail in Section 2.3. On the other hand, QoS may be implemented in IP networks through two frameworks: the integrated services (IntServ) and the differentiated services (DiffServ) [50]. IntServ may be used for implementing end-to-end QoS guarantees within an IntServ domain through the RSVP resource reservation protocol, whereas DiffServ implements class-based differentiation of traffic based on defined per-hop behaviours (PHB). The DiffServ PHBs specify the traffic forwarding principles associated with a certain traffic class between two adjacent routers. The DiffServ approach is typically selected for IP networks due to its simplicity and good scalability. Moreover, the coarse-grained QoS provided by DiffServ suffices in most IP networking and application scenarios. In heterogeneous networks, DiffServ may be used for achieving a QoS mapping between different ANs and technologies as well as implementing end-to-end QoS support [51-53].

\section{Mobility management using Mobile IP}

In order to provide a better understanding on the operation of the network-layer mobility management, MIP is introduced in more detail in this section. MIP [2, 11-13] is probably the best-known IETF standardized protocol for handling IP mobility, that is, for maintaining the reachability of a mobile node $(M N)$, whenever it changes its point of attachment to the Internet (i.e. IP address). Two versions of MIP exist, one for each IP version currently deployed, that is, MIPv4 [9] and MIPv6 [10]. In addition, there are a number of variations to these basic protocols as mentioned in the previous section. One of these has gained a lot of attention recently and is also supported in 3GPP EPC and SAE, that is, the PMIP [54]. The benefit compared to the traditional MIP is the ability to provide IP mobility support for legacy terminal devices by handling the L3 mobility management entirely in the network side, which also minimizes the signaling between the network and MN. Nevertheless, the mobility support is restricted inside a PMIP 
domain in this case. Although noteworthy and relevant for future work, the thesis will not consider the MIP variants in more detail, but focuses on MIPv4/v6.

Figure 5 illustrates the network topologies associated with the two versions of MIP. The network elements include the correspondent node $(C N)$, with which the $\mathrm{MN}$ is communicating; and two routers: the home agent (HA) and the foreign agent $(F A)$, the latter of which is present only in the MIPv4 topology. The basic operating principle in the two versions is the same: the $\mathrm{MN}$ is associated with a static globally routable home address, through which it is constantly reachable thanks to the HA. The HA is responsible of maintaining a binding between the MN's home address and the current temporary care of address ( $\mathrm{CoA}$ ) which the $\mathrm{MN}$ obtains when roaming to a foreign network. Since the HA is a router in MN's home network, the CN does not necessarily need to support MIP; it can just send all the traffic to the MN's home address, and the HA then forwards it to the MN's current location. However, if route optimization is supported, the $\mathrm{CN}$ needs to be able to receive and process binding updates from the $\mathrm{MN}$ in order to know the MN's current CoA and to direct traffic accordingly. In MIPv6, the route optimization is a built-in feature, but for MIPv4 it is relevant only in specific scenarios [55]. The benefit obtained with route optimization is avoiding the inefficient MIPv4 triangular routing depicted in Figure 5 or the reverse tunnelling [56] introduced to ensure MIPv4 operation across ingress filtering firewalls. The inefficiency originates from the additional overhead from the IP-in-IP tunnelling implemented between the MN/FA and HA as well as the single point of failure introduced in the communication path (i.e. HA). However, in MIP4 standardization, the route optimization did not progress beyond the early proposal by Perkins and Johnson [57].

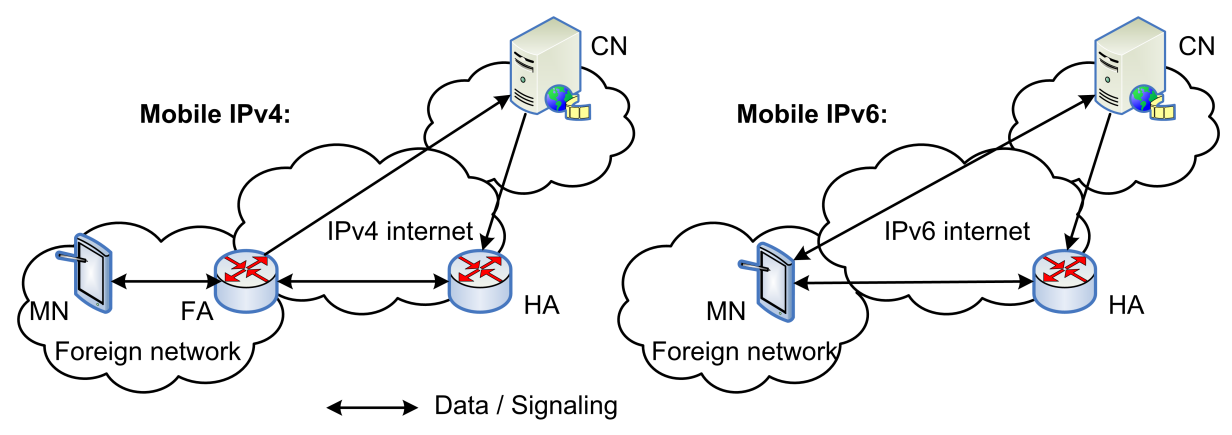

Fig 5. Network topologies in the two versions of Mobile IP. 
The biggest differences between MIPv4 and MIPv6 originate from the fact that IPv6 already provides some of the features required for mobility management (e.g. neighbour discovery and address auto-configuration), which simplify the operation of MIPv6 compared to MIPv4. In addition, the huge address space of IPv6 renders the IP address unavailability originated problems in the IPv4- and MIPv4-side (e.g. private addressing and network address translators) invalid. In MIPv4, CoA unavailability problems can be mitigated by introducing an FA in the foreign network. In this case, the MN's CoA is the IP address of the FA, and the MN can remain agnostic to most of the mobility management procedures, as the FA handles all mobility signaling and terminates the tunnels from the HA. Alternatively, MIPv4 also offers the possibility of integrating the FA functionality into the MN in the form of a co-located CoA. In this case, the MN obtains an IP address from the foreign network, for example by the means of the Dynamic Host Configuration Protocol (DHCPv4).

A handover in MIP includes two phases: handover decision and handover execution. The handover decision basically consists of mobility detection, which means that the MN determines whether it is connected to the home network or some foreign network and whether it has moved from one IP network to another, by the means of router discovery mechanisms. MIPv4 extends the Internet Control Message Protocol's (ICMP) router advertisement and router solicitation messages for implementing the agent advertisement procedure for the HA and FAs and allowing a MN to query for the HA/FAs in an IP network to which it is connected. Thus, the MIPv4 MN can detect that it has moved to a new IP network either by noticing that it is no longer receiving agent advertisements after the expiry of the lifetime of the previously received advertisement or by comparing the network prefixes on the agent advertisements to see whether the prefix on the newly received advertisement matches the previous one or not. MIPv6, on the other hand, uses the facilities of IPv6 neighbor discovery, including router discovery and neighbor unreachability detection (NUD), to determine whether it has moved from one IP network to another. As long as the last chosen default router is bidirectionally reachable, MIPv6 assumes that the chosen connection is favourable. Only when NUD suggests that the default router is out of reach, does MIPv6 start to look for a new connection.

The handover execution process is initiated once the $\mathrm{MN}$ detects that it has moved to a new IP network. The actual handover procedure depends on whether the new network is the home or a foreign network. In the latter case, the MN needs either to register with the FA serving the new network (i.e. MIPv4 with a FA) or to obtain a new CoA (i.e. MIPv4 with co-located CoA or MIPv6). Then, the FA/MN informs the HA about the 
new CoA (i.e. to update the mobility binding) as well as the CN in MIPv6. If the MN detects that it had moved back to its home network, it simply informs the HA about this.

MIP essentially realizes a brake-before-make handover, which means that there is an outage in the end-to-end connection until the new CoA is registered with the HA as well as, in MIPv6, the CN. The length of the outage is dependent on the performance of the mobility detection algorithm as well as the distance between the MN and HA/CN.

\subsubsection{Heterogeneous network management}

Heterogeneous networks and especially multi-access environments with simultaneous availability of several ANs and technologies create new possibilities for managing the constantly increasing data traffic demands over wireless. However, a great deal of complexity is introduced to networking when enabling users to roam freely between ANs, potentially, based on heterogeneous technologies and managed by separate organizations (e.g. operators, communities, private persons). Gustafsson and Jonsson [4] define a useful reference model for understanding the different aspects and players involved in an $\mathrm{ABC}$ scenario. They also define the key functionalities for an $\mathrm{ABC}$ service, namely the access discovery, access selection, AAA support, mobility management, profile handling, and content adaptation, which are addressed in part in this thesis, focusing on the mobility and content adaptation related aspects.

The definition of the best $\mathrm{AN}$ in an $\mathrm{ABC}$ scenario may depend on the requirements of the user, application or operator. Here, the management of the user experience is very important. This means that there needs to be some benefit or gain for the user from utilizing multiple ANs in an ABC scenario [4]. Depending on the user and his/her context, this benefit may, for instance, be cost, QoS or security-related; thus, impacting the criteria to be used in the access selection. The QoS requirements, on the other hand, are tied to the application and can be flexible if the application supports adaptation to network QoS. The adaptation capability is crucial to QoS-sensitive applications, as implementing end-to-end QoS in a heterogeneous multi-operator network is very challenging and no network-level QoS solutions that have this wide a scope exist today. The application-layer QoS management is discussed in Section 2.2 in the context of adaptive video streaming. Finally, the ability to steer users from one network to another provides an efficient tool for the operator to handle excessive traffic loads through load-balancing [33] or off-loading [34]. Thus, the operator policies for access and QoS 
control or steering terminals with bandwidth-hungry applications to other cells or ANs are important tools for heterogeneous network management.

There are different options for implementing the decision-making for heterogeneous network management. For example, in handover management, different approaches can be supported, including the mobile-controlled handover (MCHO), mobile-assisted handover (MAHO), network-controlled handover (NCHO), and network-assisted handover (NAHO) [58]. In the non-coupling inter-working scenario considered in this thesis, the choices for realizing the handover control are clearly the MCHO and NAHO, as they leave the ultimate control to the mobile terminal $[58,59]$. However, for instance, the MCHO scheme supported in the standard MIP includes neither optimal timing of the handover execution nor intelligent AN selection, as the handovers between ANs take place whenever the $\mathrm{MN}$ roams out of the coverage area of the currently used AN. If there were multiple ANs available simultaneously, this type of handover management would be unable to utilize the available network resources efficiently, as there is no comparison between the actual characteristics (i.e. bandwidth, delay, cost, etc.) of the networks [13]. Approaches for implementing enhanced control for MIP handovers and video adaptation will be discussed later in this thesis.

\subsection{Adaptive video streaming}

This thesis focuses on finding solutions to better support video streaming services in heterogeneous network environments and in the presence of mobility and wireless link quality fluctuations. This section presents the relevant technologies for the implementation of adaptive video streaming services with references to the literature. First, the video streaming architecture and its components and operation are introduced. Second, an overview of the SVC technology is given, with a discussion of its impact on the video streaming architecture. Finally, this section ends with a discussion of the different tools available for application layer QoS management to support video streaming services better in the best effort networks of today's Internet.

\subsubsection{Video streaming system architecture}

The video streaming system architecture considered in this thesis is depicted in Figure 6. The dissertation focuses on studying the streaming of pre-encoded videos; thus, the encoder application has been omitted from the figure. Otherwise, the architecture 
depicted contains the main components of network video streaming systems as listed by Girod et al. [6], namely the video server application storing the off-line compressed video streams and transmitting them on-demand to clients; a transport mechanism delivering video packets between the server and client; and the client application handling the decompression and rendering of the received video while providing a control interface towards the user. This chain operates in real-time, that is, a video packet containing a decodable picture received from the server is played out immediately by the client, or at least after a minor buffering delay (say, 1 second) to alleviate transmission effects (jitter) on the playout.

The network in Figure 6 consists of multiple networks of heterogeneous technologies. The video streaming client is connected to the network over wireless link(s). The video streaming may thus experience packet loss due to network congestion caused by limited wireless link capacity or other traffic; or packets may arrive past their playout deadline to the receiver causing them to be dropped. To alleviate transmission problems, Figure 6 also includes a rate control module in the video streaming server (VSS) that dynamically adjusts the video transmission rate based on client feedback. The rate control module implements application layer QoS when the network does not support QoS guarantees for the video streaming [60]. In the case of the video streaming system considered, the enforcement of the rate controller's decision, however, needs to follow the principles of rate shaping $[60,61]$ instead of rate adaptive encoding [60] due to the lack of direct interaction with the encoder. A system implementing both rate control and rate shaping is referred in this thesis to as an adaptive video streaming system.

In principle, the video rate control functionality may be placed also to other parts of the system than merely the VSS $[17,60]$. The server-side adaptation is illustrated only as an example in Figure 6. For example, the authors of [60] define a receiver-based approach in addition to the sender-based one, where it is the client who controls the receiving rate of the video. The receiver-based approach is especially useful in the case of layered videos when the video layers are transmitted in separate IP multicast groups [60], but it may also be used for unicast transmissions to remove the monitoring burden from the server $[17,62]$. Yet another approach is to place the video adaptation function in the network in a media-aware network element (MANE) [63-65].

The protocol-level design of the video streaming system architecture depicted in Figure 6 can be summarized as follows. The design, although simplified for the purposes of this dissertation, is in accordance with the video streaming systems presented by Lamy-Bergot et al. [20] and Ransburg et al. [65]. The transport of the video packets 


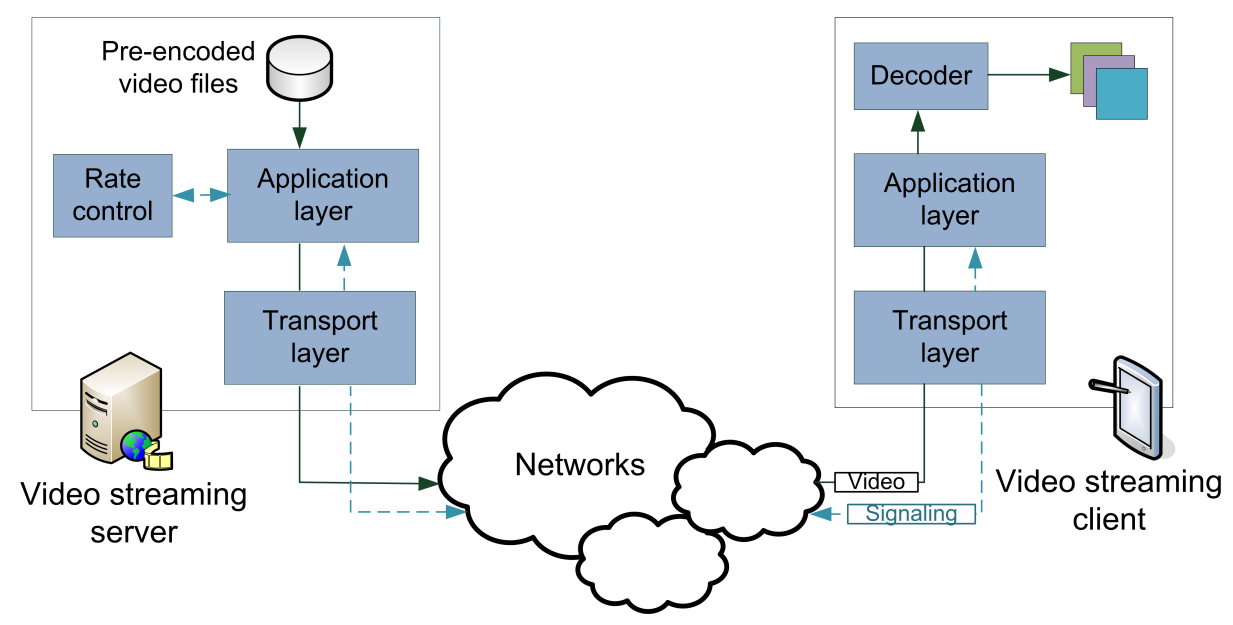

Fig 6. System architecture for streaming pre-encoded videos.

across the network is accomplished through the Real-time Transport Protocol (RTP) [66] and User Datagram Protocol (UDP) that are well suited for the transmission of streaming video. RTP is operating on the application layer and UDP on the transport layer. In addition, the Real Time Streaming Protocol (RTSP) [67] is used for session management and the Session Description Protocol (SDP) [68] for communicating the media descriptions from the server to the client at the beginning of the session in the application layer. In the network layer, either IPv4 or IPv6 is used, depending on the implementation. The signaling depicted in Figure 6 encompasses the client-server control messaging over RTSP as well as the feedback signaling from the client regarding the video streaming performance as defined by the RTP Control Protocol (RTCP) [66]. In the VSS, the feedback information is passed on to the rate control module and used for calculating a target transmission rate to be employed in the video adaptation.

The video streaming system architecture presented and its protocol-level design are generic in the sense that they do not pose limitations on the video coding technology. Only the rate shaping function is video coding technology-dependent [61]. Nevertheless, in the context of this thesis, the focus is on considering the SVC extension of the H.264 Advanced Video Coding (AVC) standard [69] when implementing adaptive video streaming services. 


\subsubsection{Impact of scalable video coding}

This section gives an overview of the SVC technology that was selected as the basis for the video adaptation solutions developed in the thesis. In addition, the impact of SVC on the video streaming system architecture is discussed in this section.

\section{Overview of the scalability extension of H.264/AVC}

SVC is the scalability extension of the H.264/AVC standard and is defined in its Annex G [70] in a joint effort of the Joint Video Team of the ITU-T VCEG and the ISO/IEC MPEG. SVC was selected as the video coding technology for this dissertation primarily due to its built-in features, which are optimal for adaptive video transmission, especially, in heterogeneous network environments [69]. With SVC, it is possible to remove portions from an encoded video bitstream without jeopardizing decodability in order to obtain different presentation and transmission properties for the same video. Since no re-encoding process is required, this makes the technology well-suited for the rate shaping type of video adaptation. All in all, SVC supports scalability in three dimensions [69]:

- Temporal scalability: Allows removing entire pictures from an encoded SVC bitstream in order to obtain a lower frame rate (e.g. from $30 \mathrm{fps}$ to $15 \mathrm{fps}$ ) and save transmission resources while maintaining decodability of the video. Temporal scalability is already supported in AVC, and SVC only provides enhanced signaling capabilities to facilitate the adaptation.

- Spatial scalability: Enables including multiple resolutions (e.g. CIF (352x288) and 4 CIF $(704 \times 576)$ ) of the same video in an SVC-encoded video bitstream to support heterogeneous terminals. So-called inter-layer prediction is supported in order to improve coding efficiency, that is, information at lower resolutions may be used to predict information at higher ones.

- SNR or quality scalability: Allows incorporating different levels of fidelity in an encoded video bitstream with a certain picture size in order to support multiple bitrates for the streaming. Here too, inter-layer prediction is employed to improve the coding efficiency. Moreover, different degrees of scalability (i.e. medium- or coarse-grain) can be supported to obtain different levels of adaptation flexibility, error robustness as well as coding efficiency, depending on the number of bitrates to be supported. 
In SVC, each feasible combination of the above three characteristics is called $a$ layer. Specific identifiers are used for defining the scalability level in each dimension, that is, Dependency ID (DID) for the spatial scalability, Quality ID (QID) for quality scalability, and Temporal ID (TID) for temporal scalability. This group of three is also abbreviated to 'DQT' in the dissertation. In SVC, the layers have a hierarchical structure and the layer that is taken as a reference for all the enhancements is called the base layer $(B L)$ with a DQT 0 . The BL is always required for decoding and it is also backwards compatible with H.264/AVC. On top of the BL, one may incrementally add one or more enhancement layers $(E L)$ to increase the video resolution, frame rate or quality (e.g. to have first a DQT of 010, then 011, and finally 012 after adding two temporal ELs on top of the BL plus one quality enhancement while keeping the picture size constant). The DQT thus defines the operation point for the SVC adaptation controller so as to keep track of the layers being sent out and to instruct a filter module to discard layers accordingly [65]. Nevertheless, there are also restrictions to the layer filtering. For instance, only the filtering of medium-grain quality enhancements can be started at every picture thanks to mechanisms limiting or eliminating decoder drift; in the other scalability dimensions, adaptation is supported only at group of pictures (GOP) boundaries [65, 69].

Being an extension to AVC, SVC follows the same principles of splitting the video coding and transmission into separate logical layers as in AVC, that is, the Video Coding Layer (VCL) and the Network Abstraction Layer (NAL). The role of the VCL is to create a coded representation of the source content, whereas the NAL formats the VCL data and adds header information so as to facilitate its transmission and use in a variety of systems [69]. Since the thesis focuses on finding solutions for improving pre-encoded video transmission in heterogeneous wireless networks, most aspects of the VCL are left outside the discussion, and the interested reader is directed to [69] for more information. The NAL, on the other hand, provides the required tools for scalable video adaptation a key functionality for this thesis - and is thus discussed in more detail in the following.

In the NAL, the SVC-coded video data from the VCL are organized into NAL units (NALU). The NALUs are packets that start with a one-byte header, which is used for signaling the type of data included in the packet and is followed by the payload. Different types of NALUs are supported by the standard, namely VCL NALUs, containing coded slices or coded slice data partitions, and non-VCL NALUs that carry associated additional information [69]. For VCL NALUs containing SVC data, the NALU header is extended by three bytes so as to carry information regarding 
the layer to which the NALU belongs (i.e. the DQT values, etc.). Of the non-VCL NALUs, the Supplemental Enhancement Information (SEI) NALUs are useful for adaptation purposes $[65,69,71]$. They are not required for decoding, but carry additional information such as the bitrates and other characteristics of the SVC bitstream as well as the contained layers.

\section{Transmission of SVC bitstreams}

In the video streaming system architecture considered in the thesis, the SVC NALU stream is directed to RTP and onwards to UDP for transport across the network. The RFC 6190 [72] specifies a specific RTP payload format for SVC supporting different mechanisms for NALU transmission. RTP adds the generic RTP payload header on top of the NALU header, which then co-serves as the payload specific RTP header to allow access to SVC-specific data also at the RTP level. Three payload structures are supported for SVC, that is, an RTP packet may carry a single NALU, several smaller NALUs aggregated in the same NALU, or a fragment of a large NALU. The last two are signaled by adding an additional information to the payload specific RTP header. This thesis does not consider NALU aggregation, but due to network MTU limitations, NALU fragmentation needs to be supported if the limitation has not been taken into account in the NAL packetization, and fragmentation on lower layers is to be avoided.

The allocation of SVC NALUs to RTP packets is illustrated in Figure 7, and the RTP payload format for fragmented NALUs in the FU-A mode [72] is shown in Figure 8. In the case of SVC NALUs, a FU-A consists of a one-byte FU-A indicator, the NALU header, and the fragmentation unit (FU) payload. For SVC adaptation handling RTP packets or even lower protocol layer ones, the NALU fragmentation poses challenges in the sense that the SVC extension header is considered to belong to the payload, thus carried only in the RTP packet containing the first fragment.

The RTP payload format for SVC also supports different transmission modes for an SVC stream, namely single session transmission (SST) and multi-session transmission (MST) [72]. This adds great flexibility to SVC transmission, as it is possible either to transport the SVC layers in a single RTP session in the SST mode or to split them across multiple RTP sessions in the MST mode. Depending on the scenario, an RTP session may carry the SVC BL, one or more SVC ELs, or the BL and one or more ELs. The SST mode is mainly intended for point-to-point unicast applications, whereas the MST mode should be used for multicast sessions where the receivers are able to 


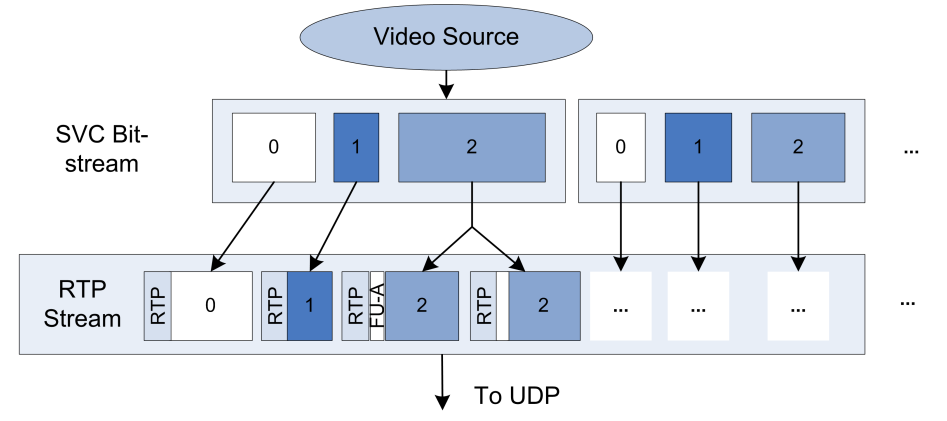

Fig 7. RTP packetization of a three layer SVC bitstream with support for FUs.
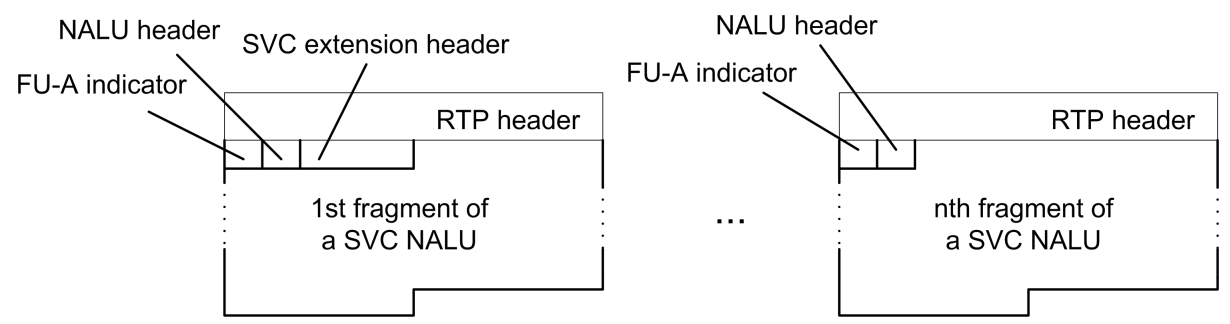

Fig 8. RTP payload format for SVC NALU fragments in the FU-A mode (modified from [73]).

request different layers of the scalable bitstream depending on their capabilities. Details regarding the different MST scenarios and the issue of decoding order recovery can be found from [63, 64] and [74], respectively.

In the video streaming system architecture, SDP is used for communicating the video session and content specific description from the server to the client at the beginning of the session. Since the introduction of SVC, SDP has also received extensions to better support layered multimedia as well as its transmission over multiple RTP sessions. It is thus possible to signal SVC layer specific information (e.g. bitrates) and decoding dependencies in an SDP session description, as specified in the RFC 5583 [68].

The flexible transmission and adaptation opportunities created by its scalability dimensions and RTP payload format make SVC very appealing for implementing adaptive video services for heterogeneous wireless networks. Although not depicted in Figure 6, SVC creates new possibilities for implementing especially in-network video adaptation using a MANE [63-65] or even realized on lower protocol layers. In this context, in addition to traditional application layer SVC adaptation, one solution for optimizing SVC bitstream delivery in the MAC layer is presented in Chapter 3. 


\subsubsection{Application layer QoS management}

In spite of the different QoS architectures and mechanisms developed for IP-based networks discussed earlier in the thesis, the best-effort approach still dominates in today's Internet access. This creates problems for video streams with real-time requirements, as they were originally designed with a constant data rate channel and low loss rates in mind, thus making them intolerant to the additional delay, jitter, and packet loss of best effort networks [17]. The alternative (or complementary) approach to providing network QoS guarantees is to enable QoS sensitive applications such as video streaming to adapt to the dynamically changing network conditions [17]. Although this dissertation considers a joint approach, this section focuses on discussing the issues specific to the application layer QoS management.

In general, the objective of video adaptation is to find an optimal solution for video transmission in heterogeneous end-user environments characterized by a variety of end-user terminals and networks. The adaptation solution needs not only to satisfy various resource constraints (i.e. the capabilities of the underlying network and the user device) but also user preferences that are seen to vary throughout a user's interaction $[75,76]$. The main challenge for video adaptation is, thus, how to maximize the utility or the QoE of the video presentation in such a diverse environment [75].

The available video adaptation approaches may be classified into three categories: rate adaptation, adaptation to delay, and adaptation to loss [17]. The rate adaptation is used for scaling the rate of the sent stream to match the available bandwidth in the transmission path. By regulating the video sending rate according to the network throughput, one can ensure that the network is capable of carrying the sent packets and that the application itself does not contribute to congestion. As a result, no packets are dropped in the network, and they also reach the receiver in time for their playout. However, the mechanism has limitations, as the adaptiveness can be incorporated in the stream only in the form of quality degradation [77], and the quality cannot be decreased indefinitely. The other adaptation approaches, namely delay and loss adaptation, can be achieved through buffering and retransmissions or error-resilient coding. There are limitations in the usage of the first two when applied to video streaming applications with strict real-time requirements, as they introduce delay to the video transmission and playout $[17,78]$. Thus, video streams with strict delay requirements should closely match the available bandwidth [17]. Nevertheless, even with efficient rate adaptation support, the video transmission may still suffer from packet losses due, for instance, to 
non-congestion originated transmission errors that are common in wireless networks. To alleviate the impact of such packet losses, error concealment algorithms such as the ones proposed by Uitto and Vehkaperä [79] may be applied to SVC streams.

The thesis focuses on enabling application layer rate adaptation of SVC-encoded video bitstreams in the presence of network transmission capacity limitations or congestion. Specifically, the streaming bitrate is regulated through adapting the video in the quality scalability dimension of SVC, which is an efficient tool for obtaining different bitrates for the video stream [69] while providing the best QoE performance for adaptive video [80]. Moreover, since the problem of defining acceptable boundaries for the video adaptation in QoE-wise is beyond the scope of this thesis, the basic assumption is made that the user requirements have been taken into account in the video encoding process, that is, it is assumed that the SVC BL quality is still acceptable to the user, although the full video quality would be preferred and the video can be adapted accordingly.

Finally, there are different options for implementing an algorithm for the rate control module depicted in Figure 6. A well-known rate control algorithm is TFRC [14-17], which can be used for implementing congestion control and fairness towards other Internet applications for UDP-based streams. TFRC is also standardized by the IETF [81], and it is known to provide smooth but responsive controlling without the high throughput variations that are possible with other controlling schemes [16]. TFRC relies on client feedback to adjust the transmission rate at the server-side according to the following algorithm (Equation 1):

$$
T=\frac{s}{R \sqrt{\frac{2 p}{3}}+t_{R T 0}\left(3 \sqrt{\frac{3 p}{8}}\right) p\left(1+32 p^{2}\right)}
$$

where $T$ is the intended data rate for the video stream (Bps), $s$ is the packet size (B), $R$ is the $\mathrm{RTT}(\mathrm{s}), p$ is the packet loss ratio (\%) and $\mathrm{t}_{R T 0}$ is the TCP retransmission timeout value (s). For implementation purposes, some simplifications may be obtained by using estimates for some of the parameters instead of the real values [16]. The enforcement of the rate controller's decision (i.e. $T$ ) in the case of SVC, can finally be achieved by a layer filtering function based on the scalability information carried in the NALU header as discussed in Section 2.2.2, Ransburg et al. [65], and Kofler et al. [71]. 


\subsection{Cross-layer signaling and control}

The dissertation is based on the hypothesis that delivering QoS-sensitive video streaming services in the diverse environment of heterogeneous wireless networks requires support beyond the layered communication architecture of today's IP networks. This section clarifies this idea by defining the concept of cross-layer optimization, and explaining the benefits it can bring for video streaming services in heterogeneous wireless networks. The section also presents the cross-layer signaling frameworks considered in this thesis, and discusses the issues and challenges involved in cross-layer design.

\subsubsection{The purpose of cross-layer information in the thesis}

Wireless networks with their advanced capabilities, including support for terminal mobility, and challenges, such as unreliability and limited or varying capacity, have resulted in the introduction of cross-layer communication architectures and designs [82-84]. Cross-layer communication, in principle, breaks the traditional communication architecture based on the well-known Open Systems Interconnection (OSI) or TCP/IP reference models by enabling non-adjacent protocol layers to exchange information for optimization purposes as well as extending the communication between adjacent layers beyond the standard procedure calls and responses. The demand for cross-layer architectures originates from the fact that traditional networking protocols, designed with wired networks in mind and realizing a strict layered communication architecture, are not capable of operating efficiently over the wireless medium [82]. Furthermore, heterogeneous networks and mobility create new opportunities and challenges for the operation of the protocols and applications that call for advanced support beyond the layered architecture. This section discusses the demand for cross-layer signaling in the context of the thesis.

Firstly, the dissertation considers the access diversity of heterogeneous wireless networks as one enabler for video stream optimization. In a strictly layered architecture, however, a handover between two PoAs using a lower layer protocol such as MIP leaves the application and transport layers unaware of the change, which may be significant, depending on the properties of the involved PoAs (e.g. technology, status, QoS support). For example, a vertical handover between WLAN and 3G has an impact on the network connection properties at least in terms of bandwidth and delay. However, current mobility management mechanisms make it impossible for the higher layer protocols 
to take timely corrective actions to cope with the abrupt change. This has led to the introduction of cross-layer enhanced versions, for instance, for TCP [85] and TFRC [86] to improve their performance under vertical handovers. Additionally, the handover decision-making of MIP remains unaware of the requirements of the applications as well as the capabilities of the available ANs without the support for cross-layer signaling. Ideally, the handover management should be aware of the overall networked system's status and the handover decision criteria used (e.g. cost, application requirements, user terminal or AN capabilities) should reflect it as accurately as possible [4, 58]. Cross-layer architectures can provide the required tools for handover-related signaling, as will be shown in Section 2.3.2.

Secondly, the thesis aims to utilize the inherent inequality of video frames or packets in an encoded video bitstream in optimizing the video transmission over resource-limited networks. For optimization over wireless links, the QoS architectures supported in IP networks and wireless MACs and discussed in Section 2.1, are not ideal for video traffic. This is because they enforce QoS on a per-flow basis, meaning that all packets belonging to a flow are treated according to the same QoS policy [50]. However, it would be useful to provide differentiated treatment to the individual packets during transmission instead of assigning the whole video flow to a single service class [19, 53, 87, 88]. This is because the video frames or packets contribute differently to the resulting decoded video quality (e.g. the SVC base and enhancement layer NALUs). Such packet-based QoS enforcement assumes that application layer priorities of video packets are known on lower layers, which can be realized through cross-layer signaling.

On the other hand, the application layer video adaptation, providing for end-to-end optimization, is concerned with the available bandwidth in the network path used; information, which is difficult to estimate accurately from wireless links and which can change abruptly. In addition, packet loss in wireless networks may occur for other reasons besides congestion, thus, hindering the operation of TCP-like congestion control. To improve the congestion control of transport protocols such as TCP, the IETF has specified the Explicit Congestion Notification (ECN) [89] protocol, which may be used for signaling network congestion events detected in network elements and for forcing the sender to react accordingly. ECN, however, limits the congestion information availability to the transport level, excluding the use of the mechanism in application layer rate control. The IETF has also specified another congestion management mechanism, called the Pre-Congestion Notification (PCN) [90, 91], which may be used for congestion-based admission control and flow termination in DiffServ packet networks. Considering the 
adaptive video streaming services, introduced in Section 2.2, this kind of network-side congestion information would be useful to improve their operation in wireless networks, and could be made available through cross-layer signaling.

Finally, careful consideration is required when designing cross-layer optimized systems. Despite the many advantages, caution needs to be exercised in cross-layer design so as not to jeopardize the longevity of the communication systems [84]. In addition, the amount of signaling required for the optimization may become significant, especially in the case of vertical handover management, where the controller may require vast amounts of information from different parts of the network for its decisionmaking. Should the controller be running on the terminal, a poor design might introduce significant processing and signaling overhead to the wireless terminal and link, and should thus be avoided. The cross-layer architecture and optimization mechanisms proposed for mobile video streaming services in this thesis are presented in Chapter 3.

\subsubsection{Cross-layer frameworks}

Different proposals for cross-layer design exist in the literature. For instance, Srivastava and Motani [82] and Fortuna and Mohorcic [83] provide an overview of different approaches for realizing cross-layer relations within a layered communication architecture. In addition, Srivastava and Motani [82] identify three implementation approaches for cross-layer optimization, namely direct communication between layers, a shared database across the layers, and completely new abstractions. This thesis focuses on cross-layer solutions that follow the principles of the first two, that is, those maintaining the communication protocol organization of a layered architecture. Direct communication between the layers is assumed in the case of signaling application layer video packet priorities to lower protocol layers (e.g. WLAN MAC). In this case, the packet priorities are signalled in-band in the packet headers. To simplify the processing on the lower layers and to avoid deep-packet inspection, mapping between application, network, and MAC layer priorities may be performed, as suggested for instance by Huusko et al. [19] and Pliakas et al. [53]. On the other hand, the two cross-layer signaling frameworks used in this thesis and introduced in this section, that is, the IEEE 802.21 MIH and the triggering framework, can be seen to realize the shared database concept, although MIH only in part. These frameworks were designed especially with heterogeneous networks and vertical handover management in mind, although they may be used for other purposes as well, including video adaptation management, as will be shown in 
Chapter 3. In addition to the cross-layer signaling that encompasses the cross-layer information collection and distribution within a communication system, this thesis identifies a number of cross-layer controllers. Located potentially on different protocol layers or network nodes, the role of the cross-layer controllers is to implement various decision algorithms and actions to optimize the operation of the communication system based on the cross-layer information. These controllers are introduced briefly in this section in the context of the considered cross-layer frameworks.

\section{Media independent handover (MIH)}

The IEEE 802.21 MIH [47, 48] defines a framework for standardized services that may be used for implementing generic link layer intelligence, and disseminating access link and network-related information to upper protocol layers in order to optimize their operation in heterogeneous network environments. By its design, MIH provides an efficient means for the handover information gathering in a multi-access scenario. The standard includes support for the IEEE 802 family of AN technologies, but also for those defined by the 3GPP and 3GPP2. MIH may be used for providing timely and consistent information about changes in link conditions and available ANs in addition to a set of handover or link management commands, thus supporting access technology independent handovers. Other aspects related to handover decision-making (e.g. policies and algorithms) and handover execution are beyond the scope of the standard. Only a brief introduction to $\mathrm{MIH}$ is provided here, and the interested reader is referred to $[47,48]$ for more details on the services and information offered by MIH.

The central entity of the MIH framework is the MIH function (MIHF), of which a reference model is depicted in Figure 9. Located between the link and network protocol layers, the MIHF interfaces with the different link layer technologies through a media-dependent interface (i.e. the MIH_LINK_SAP) and offers the upper layers access to link-level information through a media-independent interface (i.e. the MIH_SAP). In addition, message exchange between remote MIHFs is supported through another media-independent interface, that is, the MIH_NET_SAP.

An upper layer entity utilizing the MIHF services is called an MIH user (MIHU) and it may be, for example, some mobility protocol (e.g. MIP), cross-layer assisted transport protocol or an application running on a MN. The MIHU may also be located somewhere in the network. The MIHUs essentially make all the decisions for the handovers, the access link selection or the optimization of their internal behaviour based 


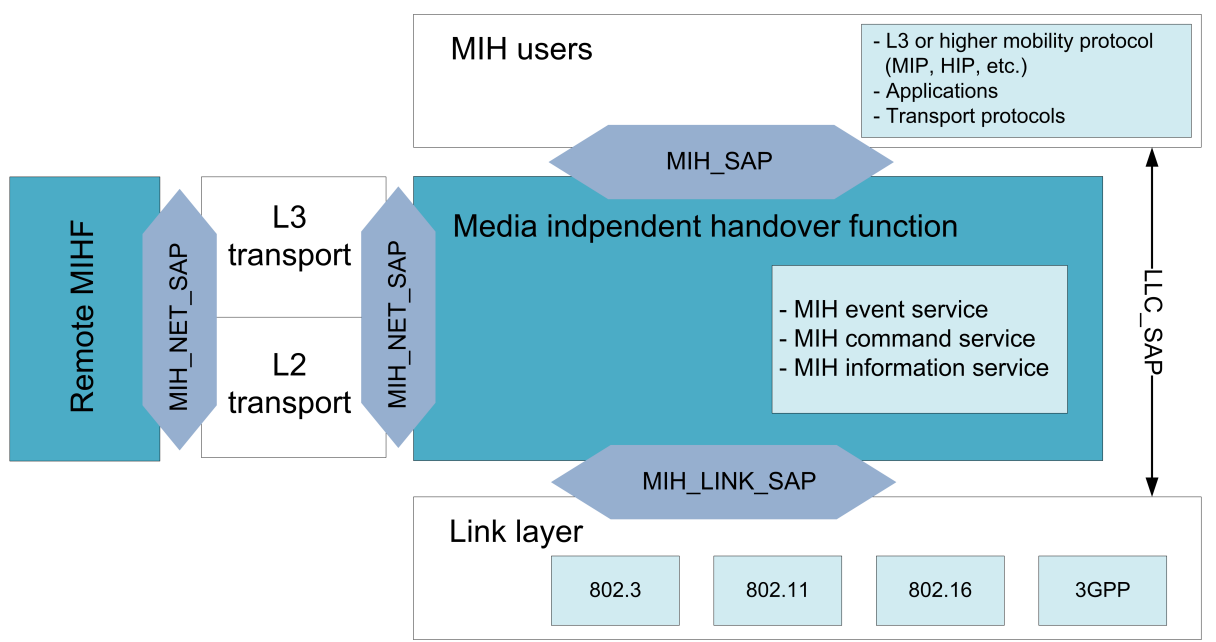

Fig 9. The reference model of the IEEE 802.21 MIH function and interfaces.

on the context information received from the MIHF; thus, they are the cross-layer controllers in the context of MIH. The MIHF offers three services to the MIHUs: the media independent event service (MIES), the media independent command service (MICS), and the media independent information service (MIIS), providing dynamic and static information on the available access links and networks as well as a means to control the link behaviour and handovers. The MIHF services may be used either locally or remotely. The communication between distinct MIHFs is facilitated through the MIH protocol run over link- (L2) or network-level (L3) transport.

\section{Triggering framework}

The triggering framework, proposed by Mäkelä and Pentikousis [49], shares a similar idea with MIH, with the exception that it supports information exchange across the whole protocol stack. As a proprietary solution, the triggering framework also allows a greater flexibility for experimenting with cross-layer designs, as it can support virtually any kind of information available in the MN or network side. Like MIH, the triggering framework was originally designed to support vertical handovers and mobility in heterogeneous networks, but since its introduction, it has been applied to other cross-layer optimization purposes as well.

The central component of the triggering framework is the triggering engine (TRG), of which a reference model is shown in Figure 10. The role of the TRG is to collect, 
manage, and distribute cross-layer information reflecting the communication system's context as formatted messages, called triggers. Within the triggering framework, the context information collection is handled through the collection interface and from various entities such as network interface cards, network monitoring tools, mobility protocols (e.g. MIP, HIP), applications, etc. The information collected (e.g. link status, available ANs and their status, QoS experienced by data transfer, etc.) is processed by the TRG according to applicable rules and policies as well as stored temporarily prior to its transmission. The TRG supports a user interface towards a user or an operator, thus, allowing them to manage the trigger distribution through rules and policies. Various consumers, such as mobility and transport protocols and applications, are able to receive timely information of the status of the overall system through TRG via the delivery interface. In addition, the consumers are able to manage the information they receive by setting rules for trigger delivery (e.g. to indicate an interest only in a specific value range of a certain trigger). A same entity may act as both a producer and a consumer to the TRG just by implementing both the interfaces. Moreover, the triggering framework may be used for an information service within the local protocol stack or between remote network entities. The latter is allowed by TRG cascading, that is making a TRG a producer or a consumer to another TRG.

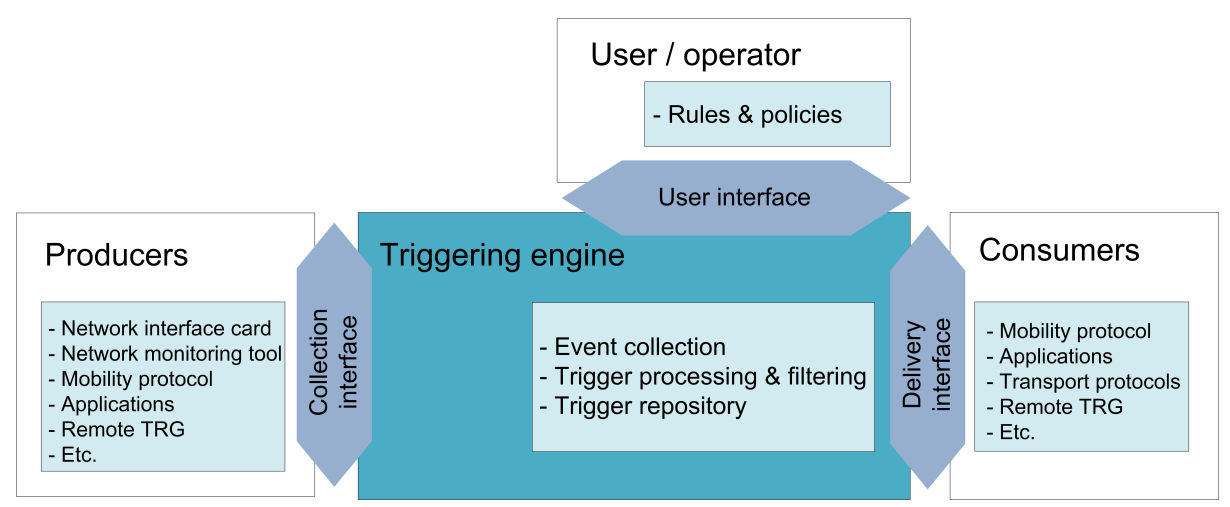

Fig 10. The reference model of the triggering engine and interfaces.

Like the MIHF, the TRG is essentially an information distribution system. That is, it leaves the final interpretation of the information received to the consumer, which then decides how to act upon it. Thus, the consumers are the potential cross-layer controllers within the triggering framework. The TRG also provides the means for the consumers to 
inform other system entities about their decision beyond the access link selection and handover decisions supported in MIH.

The user-space prototype implementation of the TRG prototype, also used in this thesis, is introduced by Mäkelä and Pentikousis [49]. The prototype supports different options for realizing the interfaces of the TRG, namely Web Service XML based communication on top of HTTP or TCP sockets with triggers formatted as TLV messages. The latter approach is adopted for prototyping in this dissertation. The interested reader is directed to the numerous publications related to the TRG concept, prototype performance evaluation, and its use in heterogeneous networking scenarios, including, for example, [49, 92-96]. In addition, some of the studies include the co-use of MIH and TRG in providing the required cross-layer signaling for optimized video transfer $[20,97]$. In this case, the MIHU is integrated as a producer for the triggering framework to distribute standardized link level information to higher protocol layers and in end-to-end scope.

\subsubsection{Controller coordination}

Both the MIH and triggering frameworks may include a number of cross-layer controllers that make decisions based on cross-layer information obtained through the MIHF and TRG. However, no coordination between the individual controllers is included. This may hamper the overall system operation and communication performance, when the controllers are run simultaneously and affect the same system parameters (e.g. work towards the same optimization goal). In cross-layer design, a holistic approach needs to be adopted instead of a fragmented one [84]. A good theoretical background for controller coordination that is partly applicable also for cross-layer controllers is established in Hämäläinen et al. [98] with a discussion of problems that arise when introducing multiple network optimization functions in a 3GPP self-organizing network (SON). Like the SON functions, cross-layer optimization can also be seen to have certain spatial and temporal characteristics that determine the impact area and impact time of the changes introduced to the system's status as a result of an action taken, although with a broader scope. Considering the cross-layer assisted vertical handover control and rate adaptive video streaming, the two operations may easily end up attempting to alleviate the same network problems, such as excessive access link or AN load, concurrently. Thus, the interactions between individual cross-layer optimization schemes need to be analysed to find possible inefficiencies or conflicts. The joint management of cross-layer 
assisted mobility and video adaptation to achieve an end-to-end QoE optimization goal is discussed in more detail in Chapter 3.

\subsection{Summary}

This chapter establishes the theoretical background for this thesis. It specifies the architectures of heterogeneous networks and adaptive video streaming services and discusses the need for cross-layer designs in their context. Related to heterogeneous networks, the main IP-based AN technologies in use today and their characteristics are introduced. In addition, the different integration models for obtaining an interworking system architecture allowing seamless utilization of the technologies are presented. For the purposes of this dissertation, the focus is on MIP-based solutions for realizing a non-coupling heterogeneous network architecture and allowing mobilecontrolled roaming between the individual networks. This chapter has also discussed the problem of heterogeneous network management in an ABC scenario, concluding that efficient management requires consideration for the requirements of different parties, including the user, application, and operator. Additionally, extensive information of the communication system's context is required, which is not supported by current solutions. In the case of adaptive video streaming, the chapter focuses on explaining the key characteristics of video streaming services as well as discussing the alternatives for adjusting the video service's QoS requirements to the network QoS. The SVC technology is introduced as an advanced video coding solution that is suitable especially for the heterogeneous network scenarios considered in this dissertation. With the introduction to the basic building blocks of a system implementing adaptive video streaming in heterogeneous networks, it is concluded that the current solutions are not capable of harnessing the full potential of multi-access networks or delivering optimal QoE for the video user; thus, justifying the use of cross-layer designs in this thesis. Finally, as a background for Chapter 3, this chapter also introduces two cross-layer frameworks and discusses the problems involved in cross-layer designs. 


\section{Contributions of the thesis: cross-layer optimization for video streaming}

This chapter presents the solutions developed in this thesis for improving video streaming performance in heterogeneous wireless networks. Specifically, it provides an overview of the results included in Papers I-V along with references to the literature and Chapter 2. The chapter begins with an introduction to the cross-layer enhanced architecture proposed for video streaming services in Section 3.1. Section 3.2 discusses the enhancements to SVC-encoded video bitstream adaptation utilizing the cross-layer architecture. Similarly, Section 3.3 presents the results regarding handover and routing optimization in the case of video streaming services in heterogeneous multi-access networks. The two approaches are combined in Section 3.4 for coordinated adaptation and mobility management. Section 3.5 summarizes the chapter.

\subsection{Cross-layer architecture for video streaming}

This dissertation takes a cross-layer approach to finding the required enhancements for supporting video streaming better in heterogeneous wireless networks. Reasoning for the selected approach was discussed in Section 2.3. This section focuses on presenting the cross-layer enhanced architecture designed for video streaming systems in this thesis as well as the related cross-layer signaling architecture. Since the thesis considers cross-layer enhanced video adaptation and mobility management as the key enablers for optimizing a video streaming service over heterogeneous wireless networks, the cross-layer architecture has been designed accordingly. Moreover, this section clarifies the relations between the cross-layer controllers coming from the original papers, as each of them has been defined in the context of a certain research project, and thus use differing terms from one another to some extent.

An overview of the proposed system architecture is shown in Figure 11. The architecture encompasses five network elements that are involved in the cross-layer optimization of the video stream transmission between the VSS and the user terminal, named as the mobile node (MN). They are introduced briefly in the following:

- Video streaming server (VSS) runs the video streaming server application and any server-side video adaptation services in the wired IP network. 
- Network information server (NIS) runs various services, such as Network Expert Systems (NES) [99], to process network-side context information prior to its transmission to MNs or network management tools. Located in the wired network, the NIS shares the same analogy with the standard information services, such as the IEEE 802.21 remote MIHF communication and MIIS as well as the 3GPP ANDSF without the restriction to handover management-related information. In larger networks, the NIS may also be deployed hierarchically for scalability [96].

- Network resource probe collects context information from the actual network equipment (e.g. PoAs and routers) within its local AN and sends it to NIS. It may also perform lightweight processing (e.g. filtering) on the information collected. In small networks, the probe functionality may be deployed in the same node as the NIS.

- Network point of attachment (PoA) is the network side end-point of a L2 link that connects the $\mathrm{MN}$ to the network and the video streaming service over some access technology (e.g. WLAN AP or 3GPP BS). It may also perform various link-level optimization functions on the video streaming.

- Mobile node (MN) runs the video streaming client application and may connect to multiple PoAs and ANs simultaneously and supports handovers between the PoAs.

Each of these network elements acts either as a cross-layer information producer generating the cross-layer information or as a consumer implementing cross-layer enhanced control functions, or as both. The cross-layer signaling between the network elements is illustrated in Figure 11. In addition, each element supports internal cross-layer signaling within the local protocol stack. Thus, the architecture facilitates distribution and utilization of extensive information regarding the networked system's status, including, for example, the available ANs and their characteristics seen by the $\mathrm{MN}$, the video streaming QoS measured in the MN, the status of PoAs/ANs including available bandwidth and congestion information and the characteristics of the video bitstream (e.g. SVC layer bitrates), as well as the priority of the video packets. Figure 11 also includes the MIP HA in the multi-access network, as it is required in scenarios where the MN uses MIP for mobility management. However, the HA is not involved in the video stream optimization in this thesis; it is only used for maintaining the binding between the MN's home address and current CoA, as explained in Section 2.1.2. The video streaming is facilitated through the RTSP/RTP/UDP protocols, as explained in Section 2.2.1 as well as in Section 2.2.2 in case SVC is used. 


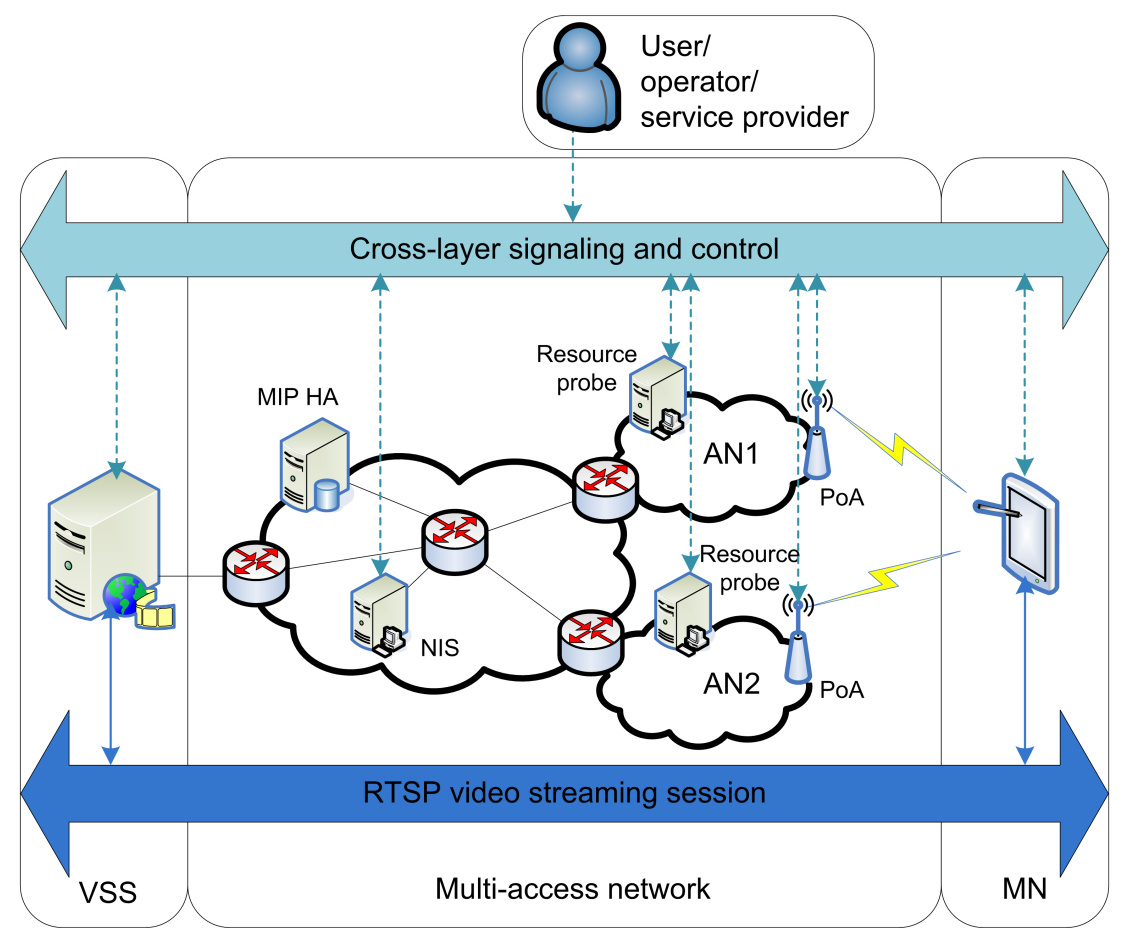

Fig 11. System architecture for cross-layer optimized video streaming.

The role of the user, operator, and service provider in managing the cross-layer signaling and control within the system is emphasized in Figure 11. This management interface is required for various security and management functions envisioned for such a system. For instance, the system should provide the means for the operator and service provider to restrict and authorize access to information regarding their networks and service. In addition, the different parties may wish to define specific operating policies in order to steer the mobility and video adaptation functionalities. Moreover, knowledge from human experts can be used in configuring the network-side probing and information processing functions (e.g. NES) to select only the relevant information for the decision-making and signaling, thus improving the scalability of the system.

An efficient signaling solution is crucial for the success of the cross-layer enhancements envisioned for the video streaming in this dissertation. Figure 12 depicts the proposed cross-layer signaling architecture. For the sake of presentation, Figure 12 omits the resource probe, which implements a suitable mechanism (e.g. Simple Network 
Management Protocol, SNMP) to collect context information from the PoA, and sends it to NIS for further processing.

The signaling is realized with the triggering and MIH frameworks introduced in Section 2.3.2. The triggering framework is used for transferring cross-layer signals both locally, that is, between entities located on the different layers of the local protocol stack, and remotely, between entities in the different network elements. Also, the end-to-end application layer feedback signaling from the MN and VSS may be facilitated through the triggering framework, or alternatively it may be realized by extending the RTCP [100, 101] or RTSP [67] protocol messages to carry the additional information. The latter would remove the need for running a TRG in the VSS. MIH, on the other hand, provides standardized link level signaling support between the MN and PoA. Additionally, an in-band cross-layer signaling method is included between the VSS and PoA to efficiently facilitate link-level video stream optimization in the PoA.

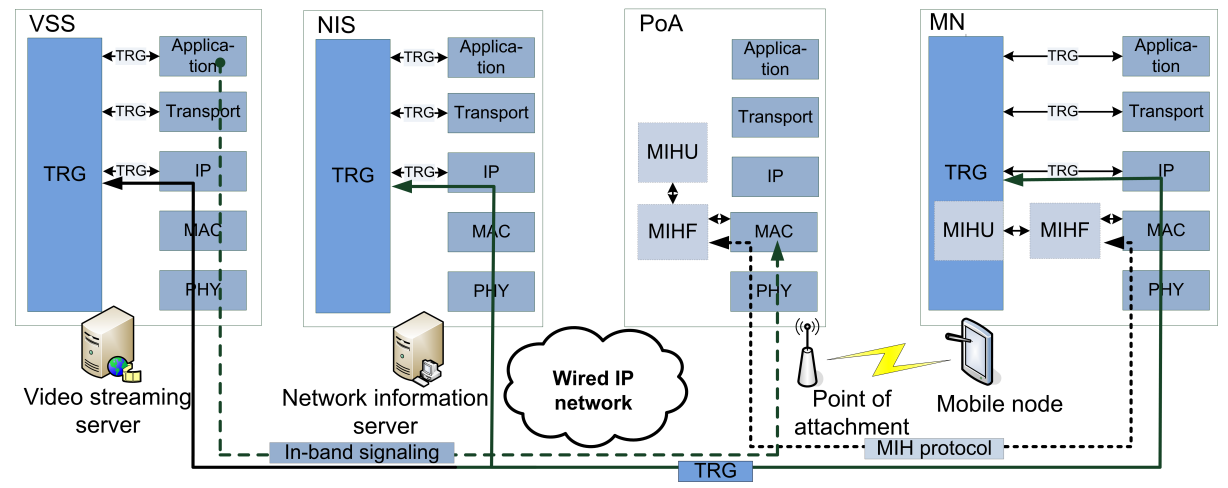

Fig 12. Cross-layer signaling architecture for mobile video streaming.

Each of the original papers included in this thesis utilizes the cross-layer system architecture for its own purposes and may consider only a subset of the included functionalities. For instance, the MIH-based signaling between the MN and PoA, shown in Figure 12, is implemented only in Paper II. In the other original papers that rely on link level information in the $\mathrm{MN}$, the information collection is handled through the triggering framework. In addition, the in-band signaling is used only in Papers I and II and the end-to-end signaling between MN and VSS in Papers II and V. The NIS and resource probe, on the other hand, are present in Papers III and V, whereas Paper IV considers mainly of MN-local cross-layer signaling. 
The original papers propose a set of cross-layer controllers for optimizing video streaming in their respective networking scenarios. The different solutions are introduced in Sections 3.2-3.4, and only a short summary is included in Table 2. The table is organized so that it includes the optimization goal, the location of the controller, and the module implementing the controller functionality, whose name may be original paper-specific as indicated in the table. As shown in the table, this thesis proposes cross-layer enhancements to the end-to-end video adaptation, handover optimization as well as dynamic network interface selection for video streaming in a multipath scenario. Additionally, a solution is proposed for coordinating those video adaptation and handover decisions that rely on the same cross-layer events for optimal performance. Finally, hierarchical control is supported in a case where the network side context information is processed in the NIS, and only critical status and control information is passed to the $\mathrm{MN}$ across the wireless interface.

Table 2. Cross-layer controllers considered in this thesis.

\begin{tabular}{|c|c|c|}
\hline Goal & Location & Module name \\
\hline 1. MAC layer video adaptation & PoA & $\begin{array}{l}\text { Video packet classifier and } \\
\text { scheduler [Papers I and II] }\end{array}$ \\
\hline 2. Server-side application layer video adaptation & VSS & $\begin{array}{l}\text { Application controller [Paper II] and } \\
\text { ADTE [Paper V] }\end{array}$ \\
\hline 3. Handover optimization & $\mathrm{MN}$ & $\begin{array}{l}\text { MIPv4 client [Paper II] and } \\
\text { MIPv6 client [Paper V] }\end{array}$ \\
\hline $\begin{array}{l}\text { 4. Dynamic network interface selection for } \\
\text { SVC-encoded video bitstreams }\end{array}$ & $\mathrm{MN}$ & AQPE [Paper IV] \\
\hline 5. Coordinated video adaptation and handovers & MN & MES [Paper V] \\
\hline 6. Network state detection & NIS & NIS [Paper III] and NES [Paper V] \\
\hline
\end{tabular}

\subsection{Cross-layer enhancements to video adaptation}

One aim of this dissertation is to find solutions for improving video streaming performance in resource-limited wireless networks. This section focuses on discussing the ability to use video bitstream adaptation as a means of providing improved QoS and QoE for video streaming across a resource-limited wireless network. The access diversity of the cross-layer architecture is thus not utilized in this section, but the focus is rather on video bitstream adaptation. In Section 2.2, video rate adaptation was discussed as a useful tool for implementing QoS support for video services in resource-limited 
networks. Nevertheless, wireless networks pose challenges for implementing efficient video adaptation due to their rapidly varying capacity. In order to find a suitable video adaptation solution for wireless networks, this thesis considers two approaches that are presented in Papers I and II, namely MAC layer adaptation in the wireless PoA and application layer adaptation in the VSS. The former provides fast and fair video adaptation to wireless link capacity, whereas the latter is needed for end-to-end optimization. The proposed solutions both utilize the capabilities of the SVC technology.

\subsubsection{MAC layer scheduling and prioritization}

In the traditional approach of adapting video streams in the application layer (e.g. TFRC), the adaptation takes place in the server or an intermediate network node, and the decision-making relies on client feedback on the streaming performance (e.g. delay and loss). However, this very feedback-based signaling mechanism makes the application layer adaptation unresponsive to rapid wireless link capacity fluctuations. The adaptation performance is affected by the signaling delay between the client and server. Furthermore, the detection of network problems in the client is slowed down by protocol and buffering delays up to the transport layer, where the feedback information is collected. Thus, application layer adaptation cannot react to wireless link capacity changes in real-time, but should instead be used for long-term adaptation for stability.

In order to improve the QoS and overcome the deficiencies in server-side adaptation in wireless networks, several standards and related proposals for prioritizing or adapting video streams in the data link layer have been presented in recent years. When optimizing video delivery, the main problem in the MAC layer QoS architectures supported by wireless AN standards such as IEEE 802.11e EDCA, 3GPP, and WiMAX is that they all enforce QoS by offering a differentiated service based on a traffic type or class (referred to as inter-traffic class $Q o S$ ). Video traffic would rather benefit from intra-traffic class $Q o S$ differentiation where the different video packets in the same stream are treated unequally by the MAC layer based on their importance to the receiver. To address this deficiency, several proposals for enhancing the MAC layer QoS for video traffic have been presented in the literature, including, for example [53, 87, 88, 102-105]. These proposals employ selective packet discarding and prioritized transmission in order to ensure that the most important video packets get transmitted over the wireless link with the highest probability. Although the solutions provide improved video 
transmission performance compared to the standard QoS mechanisms, their effect on the QoS experienced by the other traffic is, however, overlooked in the papers.

For a durable and scalable solution to adapting video streams in the MAC layer, this thesis adopts a similar approach for video packet queueing and scheduling as defined in the recently finalized IEEE 802.11 aa standard [23]. The solution introduces a new MAC layer QoS architecture to the wireless PoA to optimize video transmission across the wireless link. The optimization is done without violating the QoS allocations set for the other types of traffic in the wireless network, hence, supporting fairness towards other (non-video) traffic. A brief overview of the proposed approach is given next, and more details can be found from Paper I.

The proposed MAC layer QoS architecture is shown in Figure 13. It essentially is a multi-queue system where different types of traffic (e.g. voice, video, best effort, and background) are mapped to their own queues. To introduce intra-traffic class QoS for video streams, the video category is extended with multiple queues of differing priorities. The number of queues depends on the level of video packet differentiation required in the system.

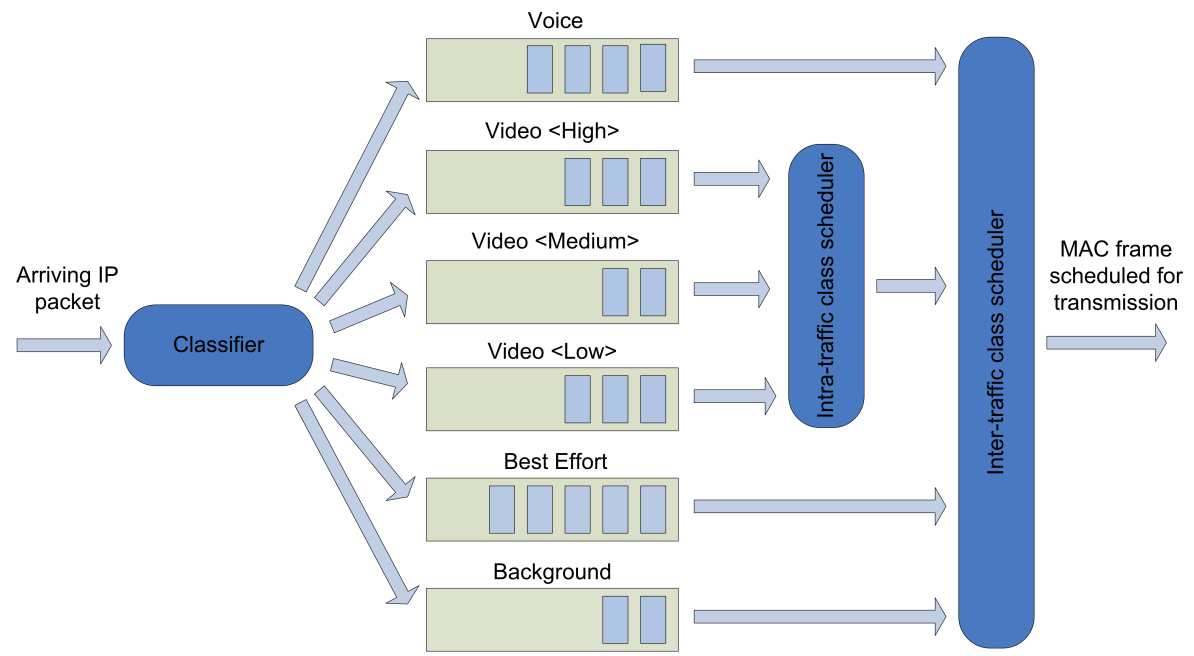

Fig 13. A hierarchical MAC layer QoS architecture supporting inter-traffic class QoS for all data types and intra-traffic class QoS for video (I, published by permission of IEEE). 
The MAC layer QoS architecture uses cross-layer information in placing the data packets to the different queues. The entity responsible for the procedure is the packet classifier depicted in Figure 13. The cross-layer information required by the classifier reveals the type and priority of the data packet, which can be obtained with in-band signaling (e.g. DiffServ), as suggested in Paper I. This signaling option is also included in the cross-layer signaling architecture introduced in Section 3.1. Alternatively, one may implement deep packet inspection in order to resolve the packet type in the MAC layer. The possibility of SVC NALU fragmentation, however, needs to be taken into account in the selected packet marking method, as the priority information is included only in the first fragment [72]. In Paper I, the video packets were split into fragments with a maximum size of 1460 bytes at RTP in order to avoid fragmentation on lower layers. The fragments were then marked according to their type.

The architecture uses two schedulers to achieve differentiated treatment both for the different types of video packets and the different types of data across the wireless link. The implementation presented in Paper I uses the standard medium access control mechanism of EDCA [22] to realize QoS-based channel access for the different traffic types. The video packet prioritization is, therefore, realized by implementing a separate video packet scheduler on top of the standard EDCA contention and collision algorithm. This second scheduler selects packets from the video queues for transmission whenever the video AC is granted access to the medium by EDCA. The video packet scheduler helps ensure video service continuity under network congestion by prioritizing packets in the high priority queues, and maximizes the QoE of the video streaming whenever the link conditions permit. In addition, since limited queue sizes are assumed, active queue management is used for removing packets first from the lower priority video queues to fit in higher priority packets under congestion.

The proposed scheme is evaluated with OPNET simulations in Paper I. The simulation study compares the performance of the proposed MAC layer QoS architecture in supporting QoS under wireless link congestion against two other approaches: the 'standard EDCA' solution as well as a cross-layer enhanced EDCA proposed in the literature and referred to as 'modified EDCA' in the following. The latter uses the four standard EDCA ACs and their single queues for video traffic differentiation in a similar manner as proposed in related works $[53,88,105]$. The purpose of the experimentation was to test the ability of the MAC layer QoS solutions to maintain QoS and QoE for the video streaming, but also for other types of traffic traversing the wireless link. The throughput of the SVC layers was used as a gauge for the QoE. The BL is always 
required for the decoding of the video and the more layers that are received the better is quality. Only a brief overview of the results is included here; more details on the simulation model, the test setting as well as the obtained results can be found from Paper I.

During the experiment, the biggest differences between the three EDCA-based video QoS support schemes were observed in the cases where the wireless link was congested and the MAC layer started dropping packets. Figure 14 shows the results measured under high wireless link congestion, under which the link is unable to support even the whole video stream. Figures 14(a) and 14(b) illustrate the data traffic throughputs measured in two clients: a video and an FTP client, connected to the same WLAN AP. Figure 14(c) shows the average packet drop rate in the different queues of the WLAN AP in the three cases. For the hierarchical scheduling, two video queues were used: a high priority queue (HPQ) and a low priority queue (LPQ), to which the SVC BL and EL packets are mapped, respectively. The modified EDCA mode places all the SVC EL traffic to the EDCA's best effort AC (AC1), leaving the video AC (AC2) to the SVC BL traffic. Thus, it performs the best in terms of SVC BL throughput of the three schemes. However, the FTP traffic, also mapped to AC1, does not go through the wireless link at all in this mode, as can be seen in Figure 14(b). The standard EDCA and the proposed hierarchical scheduling solution are, on the other hand, fairer to the FTP traffic in the sense that they do not block the FTP traffic, but only limit its throughput. Thus, they can also be considered as applicable QoS solutions for larger networks.

The fairness, however, comes at the cost of the video streaming performance. As seen from Figure 14(a), only about $20 \%$ of the SVC BL traffic gets through the wireless link in the standard EDCA mode. This is because the congestion in AC2 causes random packet drops in both the SVC base and enhancement layers. Therefore, the hierarchical scheduling performs the best in terms of fairness and video QoE. It allows sending the SVC BL over the capacity limited link without losses, when there is no other traffic besides the video stream in the link; packet loss occurs only for the EL traffic. Some BL data is, however, lost during the FTP session, but the BL transmission is restored immediately after the file transfer ends. During the period of degraded video QoE due to BL transmission problems, one option would be to search for other access alternatives for improved QoE, if the decoder cannot handle such a high packet loss in the BL. The required signaling could be accomplished through the cross-layer signaling architecture, but the actual realization is left for future work. 


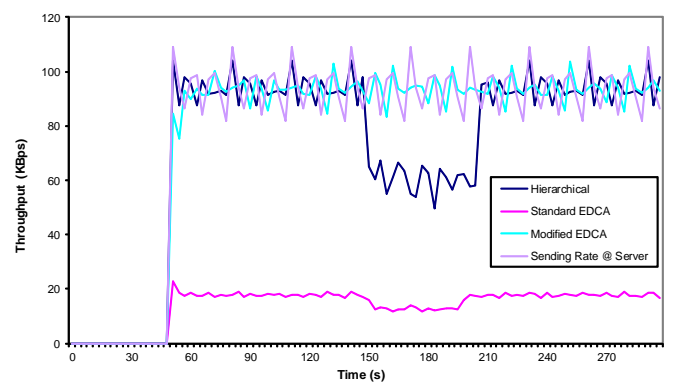

(a) SVC BL throughput measured in the video client.

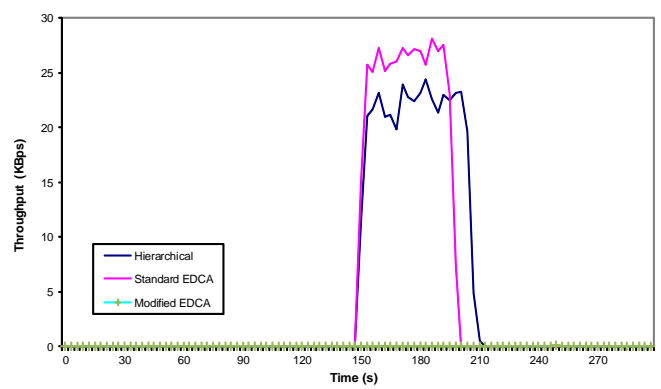

(b) TCP throughput measured in the FTP client.

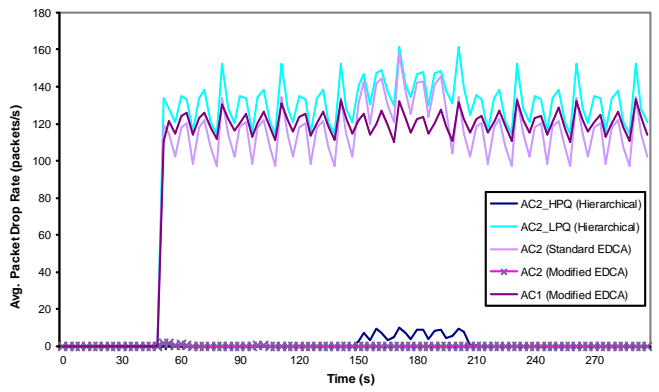

(c) Average packet drop rate for the different AP queues.

Fig 14. The performance of three EDCA-based MAC layer QoS schemes in supporting a three-layer SVC bitstream with average layer bitrates of $748 \mathrm{Kbps}(B L)$, 1.5 Mbps (BL+EL1), and 2 Mbps (BL+EL1+EL2) and an FTP download of an 1.25 MB file, starting at $150 \mathrm{~s}$, across an 1 Mbps WLAN link. (I, published by permission of IEEE). 


\subsubsection{End-to-end optimization}

The MAC layer solutions such as the one proposed in Section 3.2.1 and Paper I, however, regulate the video streaming bitrate only in the scope of the wireless link. This potentially wastes transmission resources in the wired network, as some of the transmitted data may be dropped in the PoA. Therefore, bitrate adaptation within a single protocol layer is not the most efficient way to achieve dynamic scalability in wireless networks, and it should be supported on multiple protocol layers in the proposed video streaming system.

Cross-layer optimization approaches with video adaptation support have to some extent been studied earlier in the literature. For example, a communication architecture, which utilises both application and MAC layer adaptation, is proposed by Haratcherev et al. [106]. The adaptation is accomplished by changing the transmission rate at the radio/MAC level and using cross-layer information from a low-level channel state predictor to regulate the bitrate of a non-scalable video codec. Another cross-layer approach is proposed by Huang et al. [107] for a distributed congestion control system for real-time video streaming by jointly regulating a video encoder's source rate and adapting the 802.11 MAC contention window length to control channel access for terminals. Naturally, such tight coupling of controllers located on the application and MAC layers proposed in the two papers assumes that the video source is located on a wireless terminal. This is, however, not the case in the video streaming system considered in this dissertation, where the video client is wireless. Moreover, this thesis considers the streaming and adaptation of pre-encoded videos, where the use of SVC brings clear advantage.

For this reason, it was decided to take a different approach and supplement the earlier presented MAC layer video adaptation solution (see Section 3.2.1) with application layer video bitrate adaptation. Architecturally, the functionalities are located in the PoA and VSS, respectively. Whereas the MAC layer optimization is able to react to wireless link capacity fluctuations fast, the application layer rate control is needed for handling long-term adaptation of the video bitstream in the end-to-end scope. This combined approach and the corresponding simulation study are presented in more detail in Paper II, and only a brief introduction is given next.

In the selected approach, the application layer adaptation function is realized with an entity called the application controller, which is responsible for managing video bitrate adaptation in the VSS based on client feedback. The application controller implements a TFRC-based algorithm, introduced in Section 2.2.3, for estimating the 
available bandwidth in the end-to-end path. Moreover, it utilizes the scalability features of SVC streams [69] in order to adapt the video stream bitrate accordingly.

The application controller requires feedback from the MN for its TFRC-based bandwidth estimation. In the simulation model implemented for Paper II, the application controller obtains the required information through the cross-layer signaling architecture introduced in Section 3.1. The information passed from the client to the server every $0.5 \mathrm{~s}$ contains the packet loss rate observed in the client. The reporting interval, and hence the adaptation interval, was a result of experimentation. The interval provides sufficient stability to the adaptation decisions while maintaining the responsiveness of the adaptation to network capacity changes. In addition to the client feedback, the cross-layer signaling architecture is used for enabling and disabling the video adaptation in the MAC layer dynamically based on the wireless link condition in Paper II. This is to save processing in the PoA. Three video queues were supported in the PoA for MAC layer video adaptation (when enabled), with a slightly modified video packet scheduling algorithm to the one presented in Paper I. Here too, the inter-traffic class scheduling was implemented using EDCA. The OMNeT++ based end-to-end simulator, used in Paper II and presented by Lamy-Bergot et al. [20], implements video packet fragmentation at RTP with maximum RTP packet size of 1024 bytes.

The combined cross-layer adaptation approach is evaluated with simulations in Paper II to attest its benefits. In the experiment, the scheme was compared to three other adaptation approaches, namely 'no adaptation', 'only application layer adaptation' (i.e. TFRC), and 'only MAC layer adaptation', for its performance in network resource utilization efficiency and video QoE. The evaluation was conducted under wireless link congestion causing packet drops in the PoA, which in the case of the evaluation is a WLAN AP. The network resource utilization of the different schemes was evaluated by observing the number of packets dropped in the WLAN AP during the simulation. Additionally, the Peak-Signal-to-Noise-Ratio (PSNR) was used as a gauge for userperceived video quality for its simple implementation and interpretation. Although it is a widely used tool among video researchers, PSNR has also limitations [108]: PSNR is based on a byte-by-byte comparison of the original and transmitted data without any consideration for the content. The metric is thus indifferent to video elements such as pixels and their spatial relationship as well as images and how image differences are interpreted by the human visual system. While acknowledging the limitations of PSNR, the adoption of more advanced tools is left for future work. 
Figure 15 includes a summary of the results obtained. For more results and details on the simulation model and test setting, the reader is referred to Paper II. In summary, the simulation results show that the combined adaptation approach provides the best performance when considering the two criteria: it reduces efficiently the number of useless packet transfers in the core network indicated by the low number of video packet drops in the WLAN AP in Figure 15(a) while maintaining video QoE close to the original, as shown in Figure 15(b). Thus, the results indicate that the combined approach should be used for implementing wireless bandwidth adaptation support for future ubiquitous video services. Compared, for example, to the sole MAC layer adaptation that produces a slightly better average PSNR, the combined approach reduces the number of the second SVC EL packet drops by over 50\%, thanks to the application layer adaptation capability. On the other hand, the sole application layer adaptation with the TFRC based algorithm provides barely a satisfactory level in terms of PSNR, although it reduces packet losses significantly compared to the no adaptation case (e.g. SVC BL losses are reduced by over 60\%). The weakness of the TFRC-based algorithm is its slow reaction to the decreasing channel bandwidth, which leads to packet losses before the actual application layer adaptation is triggered. Therefore, in any future development of the system, adaptation algorithms that use the wireless link status in the application layer will be considered, as suggested also by the related works. The additional feedback signaling required can already be supported by the proposed cross-layer signaling architecture.

\subsubsection{Discussion}

The results reported in this section, and in Papers I and II, show significant improvement on existing video adaptation and prioritization approaches in terms of QoE, network resource efficiency as well as fairness to other traffic. However, they also include limitations that should be considered in their application and future development. For example, the hierarchical MAC layer scheduling approach works only with quality-scaled SVC videos, as they can be adapted at every frame and not only at GOP boundaries. The quality scalability is enough for the bitrate adaptation required in this thesis, but there might be some other scenarios in which, for instance, temporal scalability is needed for a finer adaptation granularity. The MAC layer adaptation approach may also require additional support from the receiver in order to cope with possible flickering effects caused by frequent adaptations that might impair the user's viewing experience. 


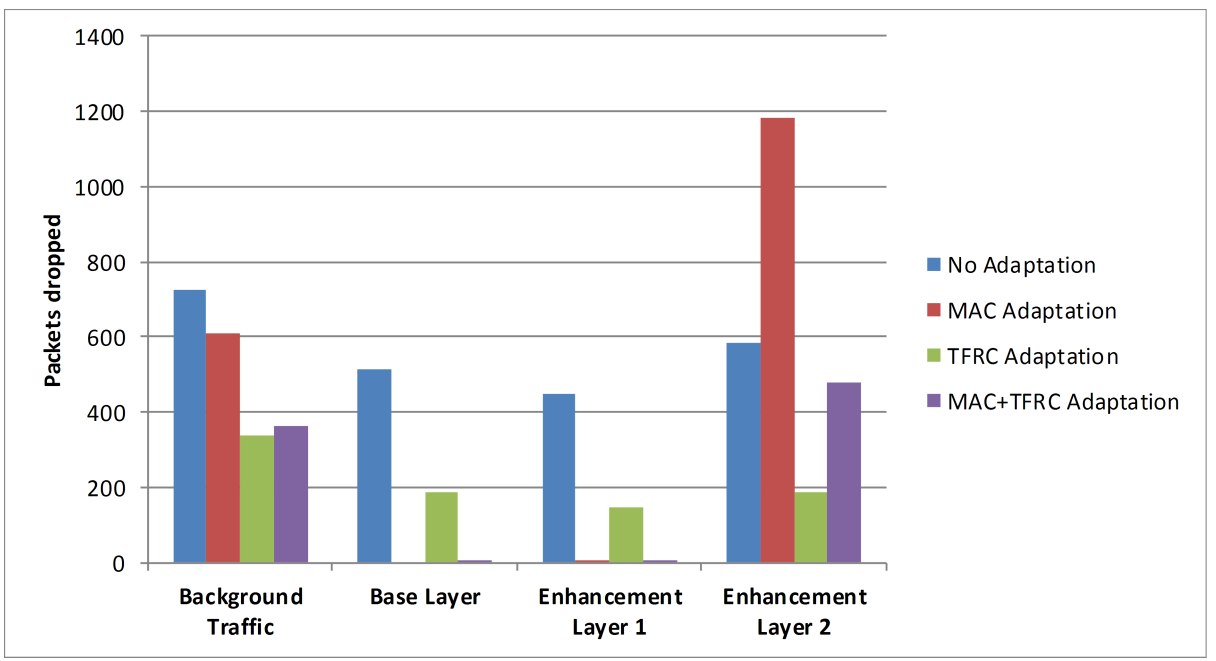

(a) Total number of packets dropped in the WLAN AP.

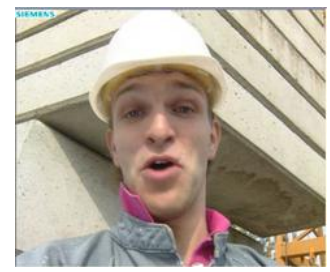

a) Original frame. PSNR $40.91 \mathrm{~dB}$.

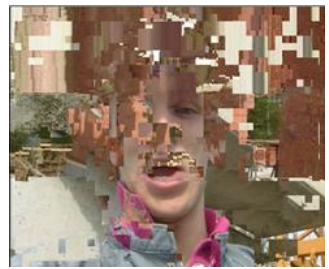

b) Reference frame. PSNR $12.88 \mathrm{~dB}$

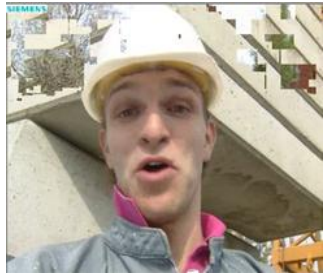

c) TFRC-adapted frame. PSNR $22.69 \mathrm{~dB}$.

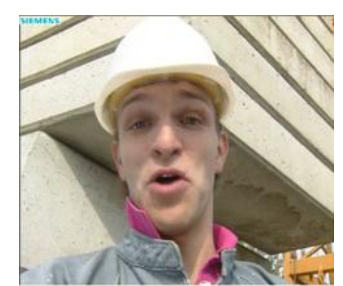

d) MAC-adapted frame. PSNR $39.21 \mathrm{~dB}$

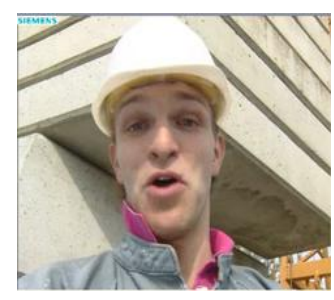

e) MAC+TFRC-adapted frame. PSNR $38.26 \mathrm{~dB}$.

(b) Visual frame quality comparison corresponding to the measured average PSNR values.

Fig 15. Simulation results for the four test cases obtained by sending a three-layer quality scaled SVC bitstream (Foreman) with average layer bitrates of $530 \mathrm{Kbps}$ (BL), $1 \mathrm{Mbps}(B L+E L 1)$, and 2.3 Mbps (BL+EL1+EL2) and progressively increasing and decreasing interfering UDP traffic with a peak rate of 2.3 Mbps across the same resource limited WLAN channel. (II, published by permission of Springer). 
Moreover, although the experimentation was limited to WLAN, similar kinds of SVC optimization support and fairness are expected to be achieved with the proposed solution per user and dedicated channel when applied to other systems like 3GPP and WiMAX. Finally, due to the poor performance of the TFRC-based algorithm under wireless link congestion, discussed in Paper II and Section 3.2.2, future work aims to study application layer adaptation algorithms that would take into account the wireless link conditions by utilizing the proposed cross-layer signaling architecture. In this respect, Papers IV and $\mathrm{V}$ consider an alternative approach to client controlled adaptation. However, no performance comparison of the approaches is included in the thesis.

\subsection{Cross-layer assisted handovers and routing}

Video rate adaptation can be used for coping with network throughput limitations, but only up to a certain degree. That is, the bitrate cannot be reduced indefinitely, but there is always a minimum below which the video QoE becomes unacceptable. For adaptive transmission of SVC-encoded videos, this minimum bitrate requirement can be considered to be that of the BL. For non-adaptive videos, the requirement is the bitrate of the full stream. It is then up to the performance of the video player and decoder used, how much packet loss can be tolerated, if the minimum throughput requirement cannot be supported by the network. This section introduces, with references to Papers III and IV, an alternative approach to video bitrate adaptation for coping with network resource limitations and congestion, that is, utilizing the AN diversity of heterogeneous multi-access networks by optimally routing the video stream. This thesis focuses on two aspects of routing optimization, namely enabling seamless usage of multiple ANs by enhancing the network-level handover performance for video streaming, and utilizing multipath video transmission when none of the available ANs is capable of supporting the whole stream. With both methods, it is possible to improve the video QoS and QoE by selecting from the available ANs the best one(s) for the streaming at all times.

\subsubsection{Mobile IP handover performance enhancement}

Although supporting session continuity across IP network boundaries, the state-of-the-art IP mobility management mechanisms, discussed in Section 2.1.2, have some limitations. The main problem, identified in the scope of this thesis, is the handover performance when considering QoS sensitive video services, discussed in this section. 
The handover decision-making of IP mobility protocols, such as MIPv4 and MIPv6, is based on limited criteria that is insufficient for timing the handovers appropriately in order to maintain sufficient QoS for video streaming applications. The agent/router discovery mechanisms supported in MIPv4 and MIPv6, and discussed in Section 2.1.2, are valid approaches for simple movement detection, but real-life situations are more complex. Mainly, the standard mechanisms fail to take into account the quality of the current connection and the possible alternatives, and nor do they consider application requirements. Moreover, even though commercial MIP implementations often also include the received signal strength (RSS) evaluation of access links, possibly enhanced with some additional static parameters such as link cost and nominal bandwidth, into their handover decision-making, the criteria still fail to reflect the actual transmission conditions such as the available bandwidth or transmission delay. This is the case especially if the network does not provide any QoS guarantees. Therefore, several proposals for enhancing MIP handover performance of MIP have appeared in the literature. For example, Åhlund et al. [109] use multipath capable MIP for comparing the relative network-level loads of the available paths and selecting the best one for use.

This dissertation proposes cross-layer triggers for mobility management in order to improve the access selection and handover performance of standard MIP to better support mobile video streaming services. The proposed approach integrates the MIP client running in the MN with the cross-layer signaling architecture, presented in Section 3.1. This allows the MIP client to obtain more extensive information on the status of the overall system and utilize it in its handover decision-making. Two solutions are considered in this thesis, one for MIPv4 and one for MIPv6. The latter is utilized in Paper V, included in this thesis and is discussed in Section 3.4. This section, on the other hand, focuses on presenting the MIPv4 based implementation. The cross-layer enhanced version of MIPv6 is, in fact, an evolution of the one designed for MIPv4, as discussed later in this thesis.

The cross-layer signaling architecture defined in this thesis enables dissemination of extensive cross-layer information between protocol layers and network nodes. For the handover performance enhancement of MIP, a set of cross-layer triggers and trigger sources have been identified, as summarized in Table 3. In terms of the system architecture, the trigger sources are located either in the local protocol stack of the MIPv4-capable MN or in the NIS, and the trigger distribution is facilitated through the cross-layer signaling architecture. The consumer for the triggers listed in Table 3 is the MIPv4 client. The NIS, on the other hand, obtains the required network context 
information from the network resource probes deployed in the ANs and monitoring the status of PoAs. In Paper III, the NIS applies simple rules (e.g. thresholds) on the information received from the probes so as to generate the triggers sent to the $\mathrm{MN}$.

Table 3. Triggers supported by the cross-layer enhanced MIPv4 client prototype (modified from Table I in Paper III).

\begin{tabular}{lll}
\hline Trigger & Source & Description \\
\hline 1. IFACE_AVAIL MN & A network interface is added or removed \\
2. IFACE_CONN MN & A network interface loses or gains connectivity \\
3. ROUTE_AVAIL MN & A route is added or removed \\
4. IFACE_FORCE MN & A network interface is to be forced \\
5. WLAN_BW NIS & A WLAN AP's current available bandwidth \\
6. WLAN_OPER NIS & A WLAN AP's operational status change \\
7. WLAN_CONG NIS & A WLAN AP's congestion status change \\
8. WLAN_QUAL NIS & A WLAN AP's radio interface quality change; a compound \\
& & variable indicating the quality in range 1-5 (low-high). \\
\hline
\end{tabular}

For the evaluation of the system, a prototype including the triggering framework, NIS, WLAN resource probe, MN-side trigger sources, and a commercial MIPv4 implementation was developed, as described in Paper III. The resource probe, specially implemented for collecting context information from the management information bases (MIB) of WLAN APs, uses the SNMP protocol for its queries. The other cross-layer signaling is facilitated using the triggering framework.

The use of a commercial MIPv4 implementation in the prototype had an impact on the decision-making algorithm in the MIPv4 client. The implementation uses network interface preference values, ranging from zero to four, for selecting the interface to use at any given moment. The interface with the highest preference value is selected for use, provided that the MN is able to connect to the MIPv4 HA through it. Thus, the new algorithm was designed to alter these preference values dynamically based on the cross-layer triggers.

Tree triggers from the NIS were used in the prototype for altering the preference values of the available network interfaces, namely WLAN_OPER, WLAN_CONG, and WLAN_QUAL as in Table 3. The first two are used to lower and restore the preference value of a network interface depending on whether their values indicate that the AP serving the interface is 'non-operational'/'congested' or 'operational'/"non-congested', respectively. The WLAN_QUAL trigger may drop or increase the preference of an 
interface depending on its value (i.e. 1-5 or 'low'-'high'). Finally, the user was given control by allowing him/her to force the usage of a certain interface by prioritizing it over the others. With this brief overview on the operational logic, the reader is referred to Paper III for more details on the prototype.

The experimental evaluation on the prototype, also presented in Paper III, was conducted in a laboratory network environment and focused on the handover performance of MIPv4 when assisted by network-side triggers. The service used in the evaluation was video streaming between a client and server with no adaptation support. The test setup is shown in Figure 16. With this setup, it was possible to test the performance of MIPv4 handovers - with and without assistance from the network - and their impact on video streaming QoS. In the setup, a mobile router (MR) running the MIPv4 client provides access to a terminal running the video streaming client through one of the two WLANs. At the beginning of the test scenario, the MR is connected to WLAN1, which is loaded with interfering traffic in 60 s periods. Whenever the WLAN1 gets too congested to support the video streaming, the MR makes a handover to the secondary WLAN2. And when the congestion alleviates, the MR returns to WLAN1. The trigger used in the evaluation was WLAN_CONG, which was set to refer to an AP load of 9 Mbps based on experimentation. The average bitrate of the non-adaptable UDP video stream was 1.44 Mbps. An end-to-end QoS measurement tool called QoSMeT [110] was used to measure the QoS experienced by the video streaming during the test.

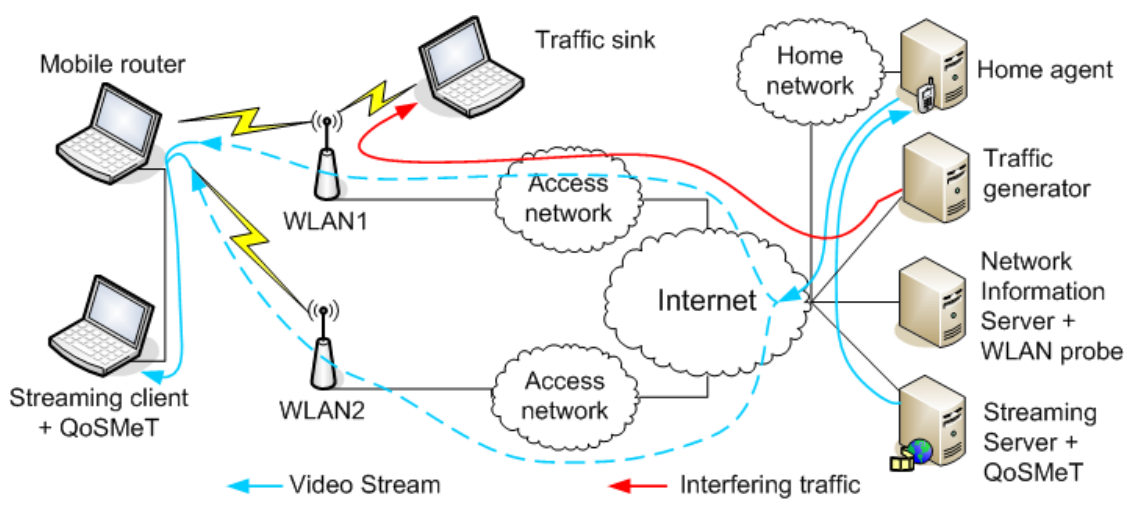

Fig 16. Network setup for evaluating the performance of network-assisted MIPv4 handovers (III, published by permission of IEEE). 
Figure 17 shows an example of the results obtained. More extensive results can be found from Paper III and Luoto [21]. Figure 17 shows the throughput of the video stream with and without using the congestion trigger for MIPv4 handovers when 15 Mbps interfering traffic is passing through WLAN1. As seen in the figure, network congestion lowers the video throughput considerably in the case of the unassisted MR. When the MR is assisted by the network, there is a short drop in the throughput after which it returns to the normal level. This is because the MR has received the congestion trigger from the network as soon as the load in the AP has exceeded the allowed level, and the trigger has caused the MR to handover to the uncongested WLAN2.

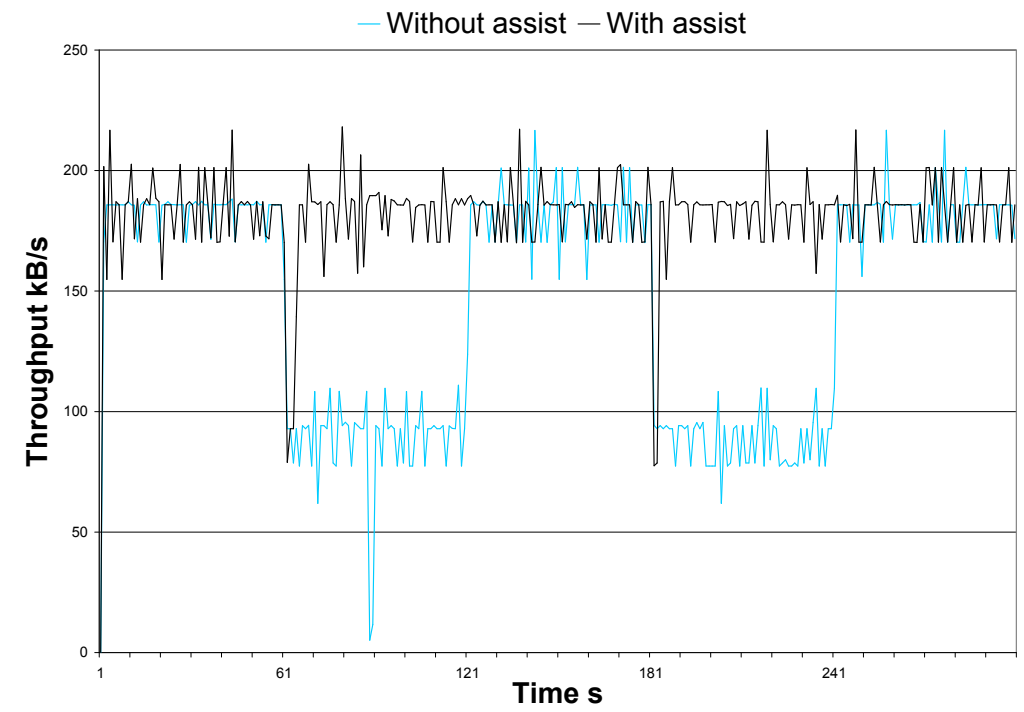

Fig 17. Video throughput measured in the streaming client with and without assistance from the network for MIPv4 handovers under 15 Mbps of interfering traffic with $60 \mathrm{~s}$ duration starting at $61 \mathrm{~s}$ and $181 \mathrm{~s}$ to WLAN1 (III, published by permission of IEEE).

Based on the results reported in this section and in Paper III, it can be concluded that the congestion trigger from the network brings a clear advantage to the video streaming QoS compared to the unassisted case. The unassisted MR does not make the handover during the congestion, since it relies only on the RSS and the periodical reregistration to the HA in its decision-making. During the tests, both of the WLANs had a similar RSS, and the reregistrations to the HA always got through the network. The approach 
was thus considered feasible, and the work was continued in Mäkelä et al. [95] by integrating more advanced and autonomous mechanisms for detecting the AN conditions and defining the Distributed Control and Management Framework (DCMF) concept for the management of heterogeneous multi-access networks.

\subsubsection{Multipath video streaming}

As discussed earlier in this thesis, several algorithms and approaches exist for implementing adaptive video streaming services. However, most of them consider video adaptation over a single network path. Similarly, the IP mobility management enhancements proposed in Section 3.3.1 focus on optimizing handovers between two networks without multi-homing support. The benefit of heterogeneous multi-access environments is, nevertheless, that they create a potential for a $\mathrm{MN}$ with multiple network interfaces to remain connected to the networked services via the best access anytime and anywhere, that is, to always stay best connected. Thus, in situations where no single AN is capable of providing the required QoS for the services used or the desired QoE for the user, simultaneous usage of two or more ANs for service delivery may be useful in order to improve the QoS and QoE. Thus, this section discusses the implementation of multipath video streaming in the context of the proposed system architecture. The discussion is based on the results reported in Paper IV.

Various solutions for implementing multi-homing with mobility support for IP hosts have been proposed in the literature. Pierrel et al. [39] enable multi-homing and simultaneous multi-access with an HIP-based solution, whereas Åhlund et al. [109] and Ciubotaru and Muntean [111] utilize simultaneous transmission over two network paths to implement seamless handovers. Andersson and Åhlund [112] use MIPv6-based flow mobility according to network-level load information in order to enable even global optimization in a heterogeneous network. However, there are not many solutions that implement cross-layer enhanced decision-making for routing SVC layers optimally in heterogeneous multi-access environments. SVC is particularly suited to multipath streaming for its support for splitting the SVC layers to separate RTP sessions and combining them at the receiver as discussed in Section 2.2.2. Related work on the concept is presented by Freris et al. [113], including a simulation study for splitting an SVC stream over multiple network connections depending on their capacity.

This thesis proposes to extend the video streaming system architecture presented in Section 2.2.1 with support for multipath video streaming, in Paper IV also referred to as 
multi-interface video streaming, as well as for the cross-layer signaling architecture from Section 3.1. The multipath video streaming principle is illustrated in Figure 18 in the context of an example scenario, in which the MN uses two networks (HSPA and WLAN) for receiving an SVC-encoded bitstream. The MN is equipped with multiple network interfaces, and both the SVC streaming server running in the VSS and the SVC streaming client running in the $\mathrm{MN}$ are able to handle scalable video delivery over multiple RTP sessions. Since the SVC layers are transported in their own RTP sessions, they can easily be routed through the different ANs to which the MN is connected by using a multi-homing capable protocol or just by making simple routing table adjustments, as suggested in Paper IV.

The proposed solution relies on the standard RTSP and SDP protocols for the signaling between the client and the server. Since a single SVC layer is transported in its own RTP session, the control of the video streaming and adaptation is different from the traditional server-side control approach. In this case depending on its condition, the client is able to explicitly setup, play, and pause individual SVC layer streams through RTSP, and the server just acts accordingly. The server-side adaptation support is needed only, if multiple SVC layers are included in one RTP session, which may be useful in some multipath streaming scenarios to reduce the protocol overhead. More details on the protocol-level design of the multipath video streaming solution for SVC and the corresponding prototype implementation are available in Paper IV.

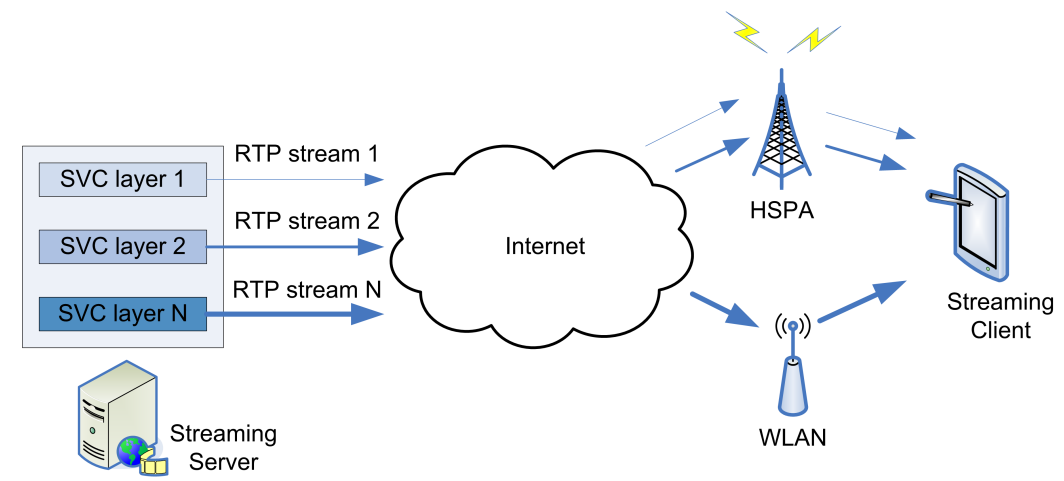

Fig 18. Multipath streaming of an SVC-encoded video (IV, published by permission of Academy Publisher).

Besides enabling the basic streaming capability across multiple network paths, the aim was to develop mechanisms for the video streaming service to cope with the 
dynamic nature of heterogeneous multi-access network environments and to adapt to changes in AN availability or capacity. For this, a new controller, referred to as the Application QoS Policy Engine ( $A Q P E$ ), is introduced into the video streaming client. The AQPE controls the number of SVC layers being received by the RTSP streaming client and uses various system status information and the characteristics of the video stream in its decision-making. For the required context information, the AQPE is interfaced with the cross-layer signaling architecture.

Different roles may be envisioned for the AQPE, depending on the realization of the multi-homing support, and whether the AQPE is integrated as a part of a distributed network management architecture such as $[95,96]$ or not. The different approaches are discussed in Paper IV. In the prototype implementation presented in Paper IV, the AQPE assumes an extensive role, and is responsible for selecting the number of SVC layers received at all times, mapping them to the available network interfaces, and finally enforcing the desired routing with routing table adjustments in the MN. Whenever there is a change in the context information, the AQPE needs to re-evaluate its decision and act accordingly (e.g. trigger SVC layer rerouting). However, rerouting of the BL in this case requires additional mobility support to ensure seamless session continuity. In addition, depending on the protocol used for RTSP transport, the AQPE may need to implement reliability mechanisms (e.g. retransmission) to ensure successful signaling.

Potential cross-layer information sources for the AQPE include network interface cards, QoS/QoE measurement tools, bandwidth estimation tools, mobility managers, etc. depending on the use case. The AQPE itself may also generate information regarding its status and distribute it to other system elements. To facilitate experimentation, the AQPE prototype in Paper IV was added with support for QoS triggers listed in Table 4. The triggers carry video streaming QoS information measured with QoSMeT [110] and they are sent either periodically or as event-based. In addition, the prototype uses the MN-local network interface and route related-triggers included in Table 3.

Table 4. QoS-based triggers supported by the AQPE prototype.

\begin{tabular}{lll}
\hline \multicolumn{1}{c}{ Trigger } & Source & Description \\
\hline 1. QOS_PERIODIC & $\mathrm{MN}$ & Periodic report of the video streaming QoS \\
2. QOS_EVENT & $\mathrm{MN}$ & A threshold set for a QoS metric was crossed \\
\hline
\end{tabular}

Finally, this dissertation includes experimentation on the multipath video streaming prototype. A brief summary of the experiments and results is included here, and more 

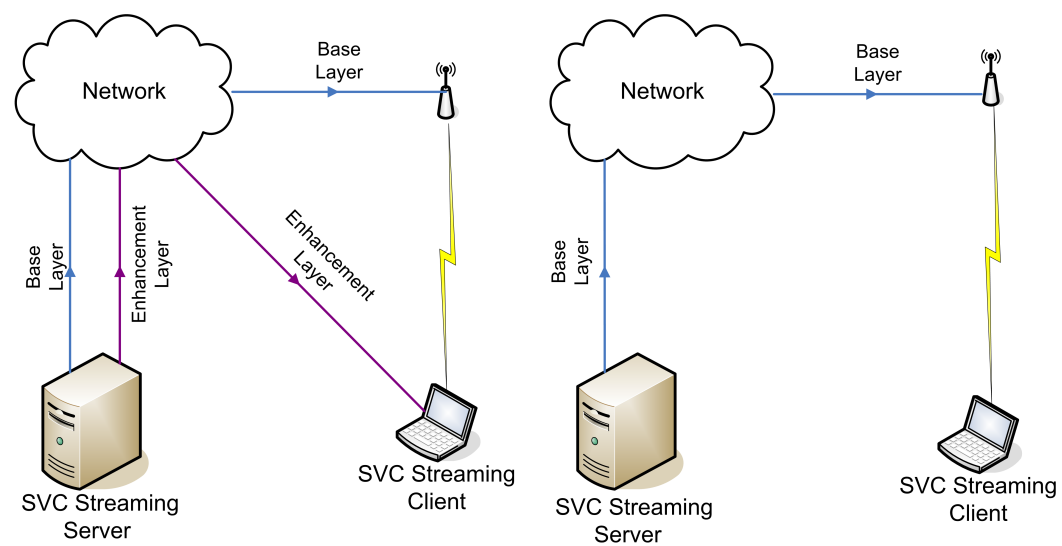

Fig 19. Test setup in the 'cable unplugged' scenario with WLAN and Ethernet access for the client (IV, published by permission of Academy Publisher).

details can be found from Paper IV. The evaluation was conducted in the context of two test scenarios, namely the 'cable unplugged' and 'limited bandwidth' scenario. The first scenario was tested with a setup, shown in Figure 19, and is described in more detail in the following. At the beginning of the scenario, the SVC BL stream is routed through a WLAN and the EL stream through a LAN towards the client terminal. This is based on an assumption that the WLAN is incapable of supporting the whole stream. At some point, the Ethernet cable is disconnected in the middle of the streaming. This is detected by the AQPE based on the ROUTE_AVAIL trigger, and it decides to stop the EL streaming for the duration of the connection outage. When the cable is plugged in again, the AQPE requests to continue the EL streaming over the Ethernet interface. In this scenario, the SVC BL streaming continues uninterruptedly through WLAN, and to the user, the LAN disconnection appears as a period of reduced video quality. The measurement of the SVC layer throughputs obtained with QoSMeT [110] in the test case is illustrated in Figure 20. The QoS triggers were tested using a similar setup in the second test scenario. In this case, the whole SVC stream is first received through the LAN, and the QoS trigger is used to detect when congestion introduced to the LAN starts causing packet loss for the video streaming. The packet loss trigger sent to the AQPE would then cause the rerouting of the EL stream to the alternative WLAN. More detailed results related to this second scenario can be found in Paper IV.

All in all, although they were obtained in a fairly simple evaluation, the results presented in Paper IV show the correct operation of the dynamic network interface selection solution implemented into the multipath video streaming prototype. In addition, 


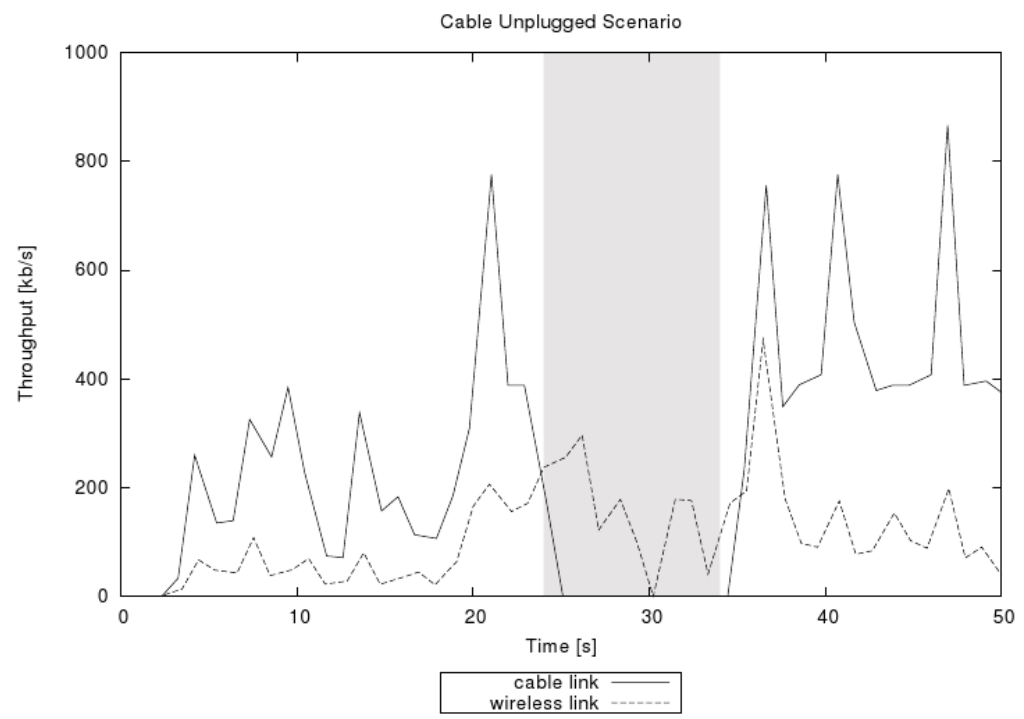

Fig 20. SVC BL and EL throughputs in the 'cable unplugged' scenario with a LAN disconnection from 24 to 34 s (IV, published by permission of Academy Publisher).

the experiments attest the potential of the multipath streaming capability to enhance video streaming performance in terms of session continuity and throughput under network impairments in a heterogeneous multi-access network. The solution may, thus, be used for improving the video streaming QoE in heterogeneous multi-access networks.

\subsubsection{Discussion}

This section, and Papers III and IV, have presented two viable solutions for optimizing handovers and routing for video streaming services in heterogeneous multi-access networks. Both of them rely on cross-layer information in implementing the enhanced decision-making for their target scenarios. The prototype implementations, however, included fairly simple decision logics, thus leaving room for future development. As stated already, the work reported in Paper III was continued in Mäkelä et al. [95] and in Paper V, which is included in this thesis. The multipath streaming concept, however, was noticed to be more challenging as current video players lack support for receiving video over multiple RTP sessions. In addition, the proprietary video player and the JSVM reference decoder used in Paper IV posed limitations in the experimentation due to their poor tolerance to transmission errors. Therefore, more advanced video streaming 
components and multipath protocols are being investigated in order to continue the development of the multipath video streaming solution. In addition, some interesting problems related to heterogeneous networks, such as advanced solutions to multipath SVC substream synchronization in the case of differing path QoS characteristics or handling the handover and rerouting decisions in a larger scale considering multiple terminals or different user applications, are not addressed in this thesis and are left for future work. The latter would require additional control and coordination in the network and $\mathrm{MN}$ for optimal performance. One possible approach for extending the proposed system architecture to support coordination and scalability in the network-side control for the management of multi-access networks has already been presented in Kliazovich et al. [96]. Related solutions are also being detailed in follow-up projects.

\subsection{Coordinated video adaptation and mobility management}

The two previous sections, that is Section 3.2 and 3.3, discussed solutions for enhancing video streaming performance under network congestion by either utilizing the video bitstream adaptation capabilities of SVC or the access diversity of heterogeneous multi-access networks. However, in Section 3.3.2 these aspects were considered together, although with less focus on video bitstream adaptation. That is, the work targeted for maximizing the video quality at all times by utilizing the available transmission capacity to the full and applying video adaptation only when necessary. However, in heterogeneous multi-access networks, there are also other characteristics and policies impacting the definition of optimal video streaming performance besides just throughput and video application requirements, as discussed in Section 2.1.3. Thus, in order to support the required flexibility in multi-access network management, this dissertation needs to consider systems supporting both video adaptation and the utilization of the AN diversity. Moreover, sufficient coordination of the applied cross-layer controllers needs to be ensured for stability and performance, as discussed in Section 2.3.3. This section provides an overview of the solution proposed in this thesis to address these issues. The interested reader is referred to Paper V for more details on the topic. 


\subsubsection{Overview of the proposed solution}

Both the SVC bitstream adaptation and MIP handover management discussed earlier in this thesis implement their own decision mechanisms in order to react to changes in wireless network conditions. For the first of these, this basically means adapting the video stream bitrate to meet the network throughput, whereas the cross-layer enhanced MIP manages the rerouting of the streaming session whenever the network to which the $\mathrm{MN}$ is connected is suffering from congestion. Nevertheless, the decisions on the different protocol layers are made independently from one another, which may lead to unoptimal operation when applied in the same communication system. This is especially true if the optimization functions are reacting to the same event, such as congestion, which is the case in the solutions considered in this thesis. To address this problem, this section introduces a new functional entity to the $\mathrm{MN}$ in order to handle both video adaptation and handover operations in a coordinated manner.

As in Section 3.3, this section also considers the case of two-tier decision-making where the network provides the MN hints regarding the status of available ANs, and the $\mathrm{MN}$ then acts accordingly. In the proposed system, this is accomplished through two controlling entities, namely the Network Expert System (NES) handling the decisionmaking and triggering functions in the NIS, and the Mobile Expert System (MES) running in the $\mathrm{MN}$ and making coordinated decisions regarding video adaptation and handovers. The signaling between the controllers as well as the associated context information collection is accomplished by using the cross-layer signaling architecture introduced in Section 3.1. The proposed solution is essentially based on the DCMF concept proposed by Mäkelä et al. [95]. Kliazovich et al. [96] extending the DCMF to support hierarchical decision-making required in multi-operator environments for better scalability, but these extensions are left for future work and not considered in this thesis.

In this case too, a prototype of the proposed system was developed and evaluated in a laboratory network environment. The prototype, presented in detail in Paper V, includes all the network elements of the cross-layer system architecture. Furthermore, cross-layer controlling is supported in the NIS, MN, and VSS, and instead of implementing a server-side adaptation algorithm such as TFRC, a client-based approach is used. The prototype supports joint control of adaptive RTSP streaming of SVC-encoded videos and network layer MIPv6 handovers through the cross-layer signaling architecture and MES. In the selected approach, the MES specifies the desired DQT values for the streaming based on the current network state and signals them to the VSS using the cross-layer 
signaling architecture. The adaptation decision-taking engine (ADTE) running in the VSS then checks the received parameter values for correctness and initiates the SVC bitstream filtering in the VSS accordingly. For the handovers, the MES instructs the MIPv6 client to move between the two networks available as defined by Mäkelä et al. [95]. Here too, the signaling is handled through the cross-layer signaling architecture.

For the network-side control implemented in the NIS, the prototype supports a more advanced solution than the one presented in Section 3.3, namely the NES. The NES and its prototype were originally presented by Horsmanheimo et al. [99]. The operation principle of the NES is similar to the simpler NIS implementation presented earlier, that is, it collects network context information for its decision-making from the network resource probes, deployed in the ANs, and generates triggers based on the information that may initiate various network management actions. The difference from the earlier version is that the NES uses cognitive decision-making algorithms, such as Self-Organizing Map (SOM) [114], for autonomous operation. With SOM, human experts have a role in guiding the decisions of the NIS, but only in terms of selecting suitable training data to be used and defining the rules for the trigger generation. The training is done off-line, and the process is described by Horsmanheimo et al. [99]. After the training, the NES is ready to be used.

In this thesis, the NES is used for detecting various conditions in a WLAN AP, such as the level of congestion or the quality of the AP's radio interface, and informing the MN when some action is required. For evaluation purposes, the same WLAN_CONG and WLAN_QUAL triggers that were listed in Table 3 were implemented in the NES prototype; now using SOM. In the prototype, these triggers are sent to the MES to inform it about the prevailing network conditions in order to allow it to initiate the correct optimization function.

For the purposes of this thesis, a prototype of the MES functionality was implemented so as to control video bitstream adaptation and MIPv6 handovers based on the network's status, reported by the NES, and any applied policies. In addition to the triggers from NES, the MES may potentially use additional information for more informed decision-making, such as the local triggers listed in Tables 3 and 4, but support for these was not yet implemented in its decision logic. For adaptive video streaming services, the MES also needs to know the minimum and maximum throughput requirements of the video stream, and whether the video is currently being streamed or not. Using SVC, the required information (i.e. the SVC layer bitrates and RTSP session status) can be obtained during the RTSP session initialisation through the SDP description, as well as 
by monitoring the status of the RTSP streaming client. The required signaling can be handled via the cross-layer signaling architecture.

All in all, by maintaining an explicit knowledge of the video streaming application status, its QoS requirements, and a set of current communication status and performance parameters, the MES is able to make informed and coordinated decisions regarding the video adaptation and MIPv6 handovers. Depending on the MN's capabilities, the MES may also implement advanced decision-making and learning algorithms like the NES [96]. However, only a simple decision algorithm was implemented into the MES prototype for the first pass experimental evaluation included in this thesis. The decision logic is described in more detail in the next section as well as in Paper V.

\subsubsection{Experimental evaluation}

The purpose of the prototype introduced in the previous section was to demonstrate the potential of the coordinated video adaptation and handover management solution in providing the required tools for efficient multi-access network management. It was built using open source components for scalable video streaming as well as for MIPv6. The components were modified so as to include support for SVC adaptation as well as the cross-layer signaling and control, and deployed into a network testbed as shown in Figure 21(a). In addition, the implementation includes the NES, MES, WLAN probe, and triggering framework components that are also visible in the figure. The prototype supports a simple decision logic for the MES to manage video adaptation and handovers in the case of a two-layer quality-scaled SVC stream and a multi-access network encompassing two WLANs. The MES logic is illustrated in Figure 21(b). The SOM-based NES was trained according to the procedure in Horsmanheimo et al. [99] and using probe data collected by introducing different levels of traffic into the WLANs, eventually congesting the APs. The probe data used included current available bandwidth, radio interface quality, and packet loss measurements. The accuracy of the training was evaluated experimentally by running the training multiple times and selecting the trained SOM with the smallest quantization error and topology preservation error for use as suggested by Horsmanheimo et al. [99]. The remainder of this section provides a brief overview of the experimental evaluation on the prototype and the results obtained. The reader is referred to Paper V for more details.

The experimentation was conducted in the context of an example scenario, where the decision-making of the MES is limited with a user policy. This policy dictates that 
the multi-access mobile terminal, also known as the $\mathrm{MN}$ in this thesis, needs to stay in the preferred AN1 network and AP1, whenever available and able to support at least the SVC BL. In the scenario, it is assumed that the AN2 is more expensive for the user (e.g. pay per bit) than AN1. If, however, the AP1 becomes congested and thus unable to support the video streaming, the MN should start looking for alternative networks and connect to one of them, given that it is able to support the minimum SVC BL requirement. In the setup used, the $\mathrm{MN}$ has both the WLANs constantly available, and thus makes a handover to AN2 and AP2 whenever needed. However, when connected to $\mathrm{AN} 2$, the MN receives only the base quality video to keep the traffic to a minimum due to the more expensive connection. The state transitions are triggered, based on network status information received from the NES as illustrated in Figure 21(b).

Figure 22 illustrates an example result obtained from a test run; more results can be found in Paper V. The QoSMeT tool [110] was used for measuring the video streaming performance between the VSS and the video streaming client (i.e. MPlayer in Figure 21(a)). Interfering traffic was injected into AP1 in order to test the operation and performance of the proposed system. Figure 22(a) shows how the video stream is adapted to lower quality after the adaptation trigger from NES at $95 \mathrm{~s}$. The trigger was caused by the increased network load as the interfering traffic increases to $11 \mathrm{Mbps}$ at $90 \mathrm{~s}$. The still increasing interfering traffic (16.5 Mbps starting at $120 \mathrm{~s})$ results in radio link congestion, also detected by NES and resulting in a handover trigger at $122 \mathrm{~s}$. This congestion also causes some video packet losses and increased delay as seen in Figures 22(b) and 22(c). While connected to AP2, the video client receives only the SVC BL, as dictated by the operating policy and seen in Figure 22(a). When the radio link quality in AP1 restores back to 5, the NES reports it to MES, which then triggers a handover back to AP1 at $153 \mathrm{~s}$. However, since the interfering traffic load is still 11 Mbps, the returning BL video traffic causes the radio interface quality to drop to three, and the client continues to receive the base quality video also in AN1. The video quality improvement trigger takes place at $184 \mathrm{~s}$, after the interfering traffic has dropped to 5.5 Mbps and the radio link quality is restored to 5.

Based on the results, it can be concluded that the proposed solution can be used for optimizing video stream delivery in multi-access networks according to a specific user- or operator-defined policy. Moreover, the evaluation and prototype presented are steps towards implementing the mobile video streaming systems of the future, allowing efficient control over video bitstream adaptation and user terminal mobility. 


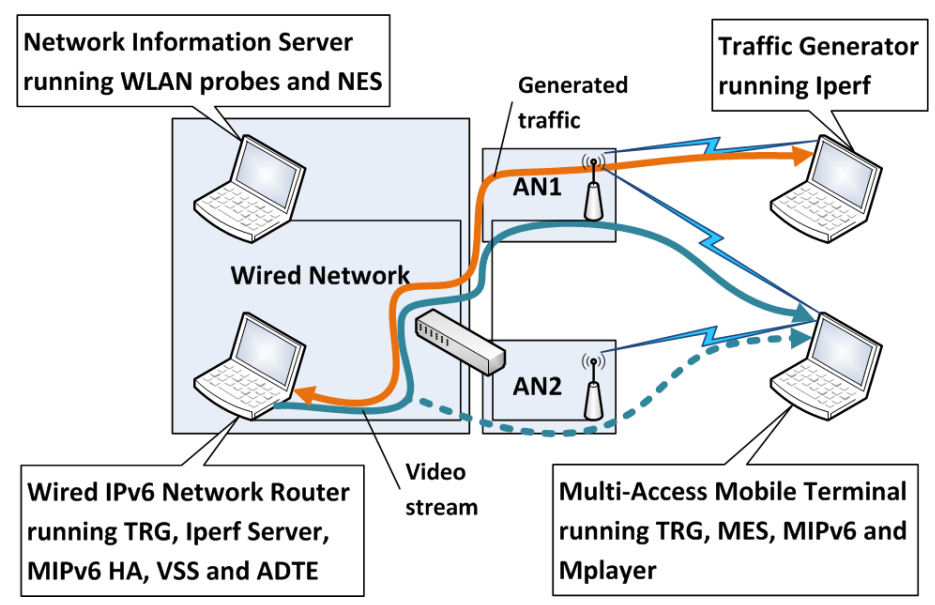

(a) Network setup of the prototype.

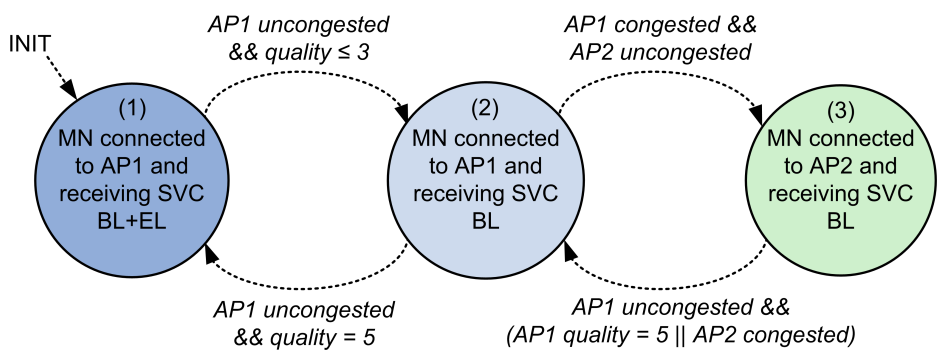

(b) State diagram for MES prototype operation under the set policy.

Fig 21. Illustrations of the prototype implementation and logic for coordinated video adaptation and handover management $(V$, published by permission of Springer). 


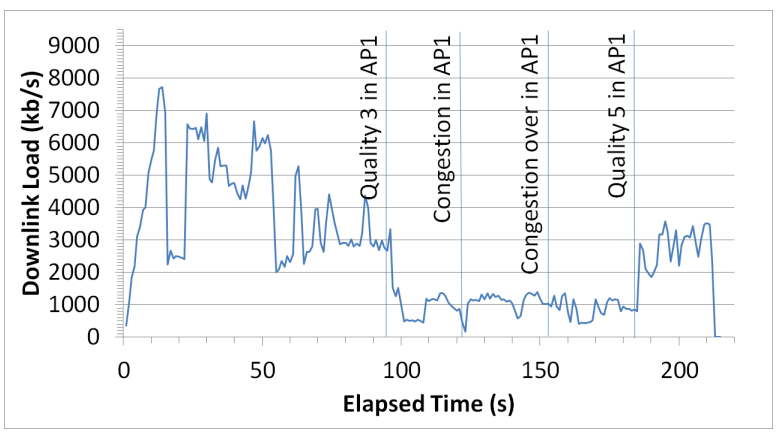

(a) Video throughput and the adaptation/handover triggers.

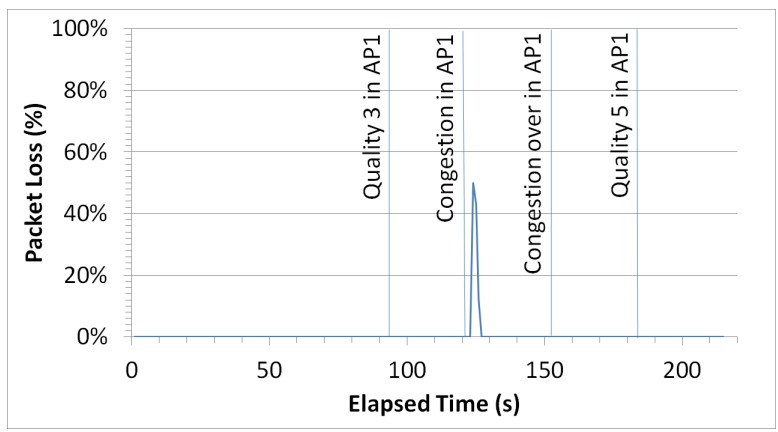

(b) Video packet loss and the adaptation/handover triggers.

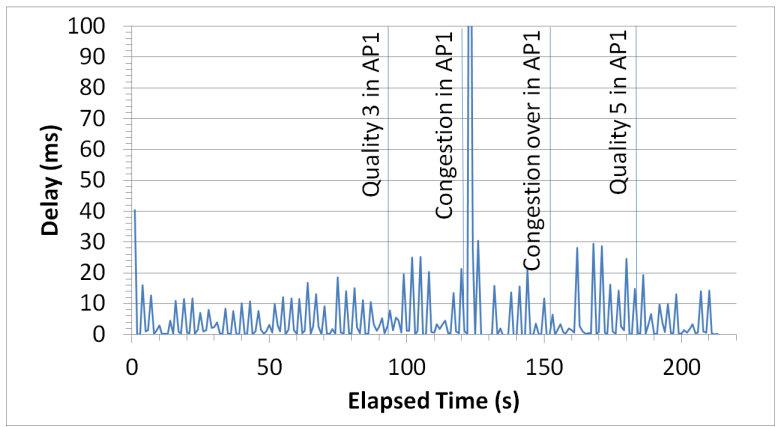

(c) Video packet delay and the adaptation/handover triggers.

Fig 22. The performance of the multi-access management in supporting a twolayer SVC bitstream with average layer bitrates of $871 \mathrm{Kbps}(B L)$ and $3.8 \mathrm{Mbps}$ $(B L+E L)$ under pyramidal interfering traffic introduced to AP1 at 1 min with a step size and duration of $5.5 \mathrm{Mbps}$ and $\mathbf{3 0} \mathrm{s}$, total duration of $2.5 \mathrm{~min}$, and peak rate of $16.5 \mathrm{Mbps}$. (V, published by permission of Springer). 


\subsubsection{Discussion}

This section and Paper V presents a viable solution for joint management of video adaptation and handovers in multi-access networks based on the cross-layer architecture presented in Section 3.1. With the NES approach, this section also takes a step towards more autonomic and cognitive network management, which is envisioned as an important future development direction for the presented solution. The experiments, nevertheless, reveal weaknesses in the prototype implementation especially in the decision-making algorithms used. The main problems arise from the inability of the SOM-based algorithm to detect network conditions accurately in a dynamic network environment including real wireless links and a variable bitrate video stream. This results in a ping-pong effect in the video adaptation and handover decisions, reported in Paper V. The ping-pong effect as such poses little disturbance on the video QoE, but it generates additional signaling overhead, and is therefore undesirable. For this reason, the work will be continued in a follow-up project to incorporate more advanced decision-making algorithms, possibly with learning ability, in order to improve the stability of the cross-layer control. Moreover, similarly to the solutions presented in Section 3.3, the cross-layer controllers presented in this section will be developed further to better support scalability in the context of larger networks and in the presence of multiple user terminals.

\subsection{Summary}

This chapter provides an overview of the results presented in the original papers included in this dissertation. In order to link the results with the original research questions presented in Chapter 1 of this thesis, the discussion in this section is organized in accordance with them. This chapter addresses all three problems, which were as follows, from the viewpoint of mobile video streaming services:

RQ1 How to design and implement a cross-layer signaling and controlling system in order to enable efficient and timely mobility and video adaptation decision-making in heterogeneous wireless networks?

RQ2 What kind of cross-layer optimized functions can be used to support video transmission in heterogeneous wireless networks so that it utilizes the available network resources efficiently and fairly?

RQ3 How to ensure sufficient coordination between the introduced cross-layer functions for stability and performance? 
As an answer to RQ1, this dissertation specifies a cross-layer system architecture for video streaming services in heterogeneous wireless networks in Section 3.1. The architecture includes the different components required for video stream optimization in the scope of this thesis, as well as a cross-layer signaling system facilitating the required cross-layer information transfer. The architecture enables various enhancements to adaptive video streaming services and mobility management. These are accomplished by allowing the related controllers to react to changes in network status in a timely manner, considering the requirements of the video application, or optimizing the usage of the available network resources by ensuring successful transfer of at least the most important data. The enhancements essentially rely on the availability of extensive information on the networked system's status, which is enabled by the proposed cross-layer signaling architecture. Yet, the scalability of the solution is considered in terms of limiting the signaling overhead, especially, across the wireless link.

Regarding RQ2, this chapter provides an overview of the different solutions and algorithms, proposed in the original papers included in this thesis for cross-layer optimized video streaming in heterogeneous wireless networks. The main focus is on cross-layer controlled video rate adaptation and routing/handovers related solutions. The fairness aspect of the solutions towards other users and applications is considered mainly in the context of the cross-layer video adaptation approach presented in Section 3.2. The used adaptation algorithms are selected so that the video traffic does not consume network resources beyond its allocated or fair share. In this respect, the results presented in Sections 3.3 and 3.4 are more limited, as they do not include optimization across multiple users, which would require additional support from the controllers. However, all the solutions introduced can be seen to improve the efficiency of network resource usage. With the cross-layer adaptation approach it is possible to match the video streaming rate to the available network capacity, end-to-end, while prioritizing the transmission of video packets based on their importance to the receiver. On the other hand, the enhancements proposed for handover decision-making and multipath video streaming in Section 3.3 are essential enablers in selecting the most appropriate AN(s) for the video stream transport in heterogeneous multi-access networks dynamically, thus improving the video QoE.

The coordination problem, included in RQ3, is addressed in the context of congestiontriggered handovers and video rate adaptation in Section 3.4. Both the functions are required in the same system in order to support greater flexibility in multi-access network management for an $\mathrm{ABC}$ service. Thus, to solve the coordination issue, a new entity is 
introduced into the MN that is responsible for making joint decisions regarding the handovers and video bitrate adaptation as well as triggering the respective actions based on cross-layer information.

The solutions presented give the network operators and service providers the means for managing the QoS and QoE for their customers. Such management is becoming ever more important with the increasing data traffic loads demanded of wireless networks. The adoption of the solutions is, however, hindered by their reliance on novel components and protocols for cross-layer signaling and control. The full end-to-end solution also introduces changes to almost the whole video stream delivery chain, including the servers, ANs, and MNs. The solutions nevertheless support gradual deployment to obtain at least a subset of the optimizations.

The validation of the proposed solutions is conducted through simulations and prototype implementations. A brief introduction to the conducted experiments is given in this chapter with references to the original papers for more information. The results obtained attest the feasibility and demand for the proposed solutions in enhancing the performance of video streaming in heterogeneous wireless networks, yet leaves room for further work in terms of algorithm development and more extensive experimentation. 


\section{$4 \quad$ Conclusions and future work}

Dynamic and concurrent utilization of the access networks and technologies in today's heterogeneous multi-access network environments can bring clear advantage in supporting networked applications and user requirements. The gain may be increased transmission capacity or optimization against other criteria such as cost of the connectivity. However, an always best connected paradigm of this kind introduces great complexity into the network and application management due to the diversity of the network environment. Therefore, novel mechanisms are required for supporting optimal access selection, handover decision-making, and QoS in heterogeneous networks. This is especially true when considering video streaming services due to their strict QoS requirements in terms of throughput, delay, and loss. Additionally, they potentially involve transmission of large amounts of data for long periods of time.

To address this problem, this dissertation proposes novel solutions for improving video streaming performance in heterogeneous wireless networks. The common denominator in the proposed solutions is the utilization of cross-layer information, for which the thesis presents a cross-layer architecture for video streaming systems. The architecture realizes an extensive signaling service for heterogeneous networks to support various cross-layer enhanced mobility and video adaptation functions. To demonstrate the benefits of cross-layer optimization, the thesis proposes cross-layer enhanced approaches for video rate adaptation, network-layer handovers, and multipath transmission of video for improved performance. Different aspects of video streaming performance are studied in the context of the optimization approaches, including QoS, QoE, network resource efficiency, and fairness towards other types of traffic. Finally, in order to achieve a solution that makes use of both the video rate adaptation capability and the access network diversity of multi-access environments, this thesis has considered supporting both the functions simultaneously in a video streaming system. In this case, a solution for coordinating optimization functions operating on distinct protocol layers for efficiency and improved performance has been proposed.

The work presented essentially utilizes and promotes the flexible adaptation and transmission capabilities of the SVC technology, which are clearly beneficial when implementing video services targeted for heterogeneous networks and terminals. Nevertheless, the cross-layer enhanced solutions proposed for video stream adaptation 
are not constrained to SVC-based solutions, and could be applied also with other coding techniques or transcoders. Additionally, the other cross-layer assisted algorithms proposed for mobility management and video stream routing have been designed so that they can be used with other protocols besides MIP or the proprietary application layer multipath solution implemented in this thesis. These factors are seen to improve the adoption of the concepts and ideas presented in the thesis.

The results of the dissertation provide the means for network operators and service providers to manage the QoS and QoE for their customers, which is becoming ever more important with the ever-increasing data traffic loads demanded of wireless networks. Nevertheless, the proposed enhancements are achieved with changes to the network and service architectures in terms of introducing new components and protocols for the cross-layer signaling and control. In addition, the full end-to-end solution will require changes across almost the whole video stream delivery chain, that is, to the service provider's servers and the access network equipment as well as the mobile device capabilities. Yet, performance improvements can be obtained already with a subset of the solutions, allowing a gradual deployment. Moreover, with the inter-working network architectures for $3 \mathrm{G} / 4 \mathrm{G}$ networks and WLANs becoming more common, we may also soon see commercial implementations of information systems such as the IEEE 802.21 that can be seen as steps towards a full cross-layer architecture.

The future directions of the work presented in the thesis are essentially aimed at a cognitive networking approach, as well as addressing the deficiencies identified in current work. The cognitive networking topic has already been touched upon in this thesis in terms of utilizing cognitive algorithms in detecting the access network status and controlling the terminal mobility and video adaptation accordingly. However, in the near future the aim is to develop more advanced algorithms with learning ability in order to improve the QoS and QoE of video streaming in the end-to-end scope in heterogeneous wireless networks. The aim is also to introduce coordination to the network side in order to manage a larger number of terminals. This follow-up work is already being conducted in the frameworks of the EUREKA/Celtic COMMUNE, EU FP7 ICT CONCERTO, and VTT's AWARENESS projects. Moreover, the work regarding cross-layer video adaptation will continue in terms of studying the applicability of the proposed solutions to HTTP-based adaptive video streaming and other wireless network technologies besides WLAN. In addition, further development of the multipath video streaming solution, including more extensive experimentation, will take place in the projects. Regarding the cross-layer signaling architecture, future development will 
include enhancing the security of the information transfer, which is mainly overlooked in the triggering framework, as well as conducting more extensive analysis of the scalability of the solutions. The use of HTTP streaming opens up possibilities for considering other service architectures too, such as content delivery networks (CDN) and new CDN-specific client-server protocols (e.g. ALTO). In this context, the research presented in this dissertation can be extended to cross-layer assisted server selection and service migration optimization. 


\section{References}

1. Bhagwat P, Perkins C \& Tripathi S (1996) Network layer mobility: an architecture and survey. Personal Communications, IEEE 3(3): 54-64.

2. Pahlavan K, Krisnamurthy P, Hatami A, Ylianttila M, Mäkelä J, Pichna R \& Vallström J (2000) Handoff in hybrid mobile data networks. IEEE Personal Communications 7(2): 34-47.

3. Sachs J \& Olsson M (2010) Access network discovery and selection in the evolved 3GPP multi-access system architecture. European Transactions on Telecommunications 21(6): 544-557.

4. Gustafsson E \& Jonsson A (2003) Always best connected. IEEE Wireless Communications Magazine 10(1): 49-55.

5. Cisco (2012). Cisco visual networking index: Global mobile data traffic forecast update, 2011-2016. White paper. URI: http://www.cisco.com/en/US/ solutions/collateral/ns341/ns525/ns537/ns705/ns827/white_paper_c11-520862.pdf (Visited on 11 October 2012).

6. Girod B, Chakareski J, Kalman M, Liang YJ, Setton E \& Zhang R (2002) Advances in network-adaptive video streaming. In: International Workshop on Digital Communications (IWDC '02), pp. 1-8. Calcutta, India.

7. Cermak G, Pinson M \& Wolf S (2011) The relationship among video quality, screen resolution, and bit rate. IEEE Transactions on Broadcasting 57(2): 258-262.

8. Motorola (2009). Opportunity and impact of video on LTE networks. White paper. URI: http://business.motorola.com/experiencelte/pdf/LTEVideoImpactWhitePaper.pdf (Visited on 14 August 2012).

9. Perkins, C (Ed) (2010). IP mobility support for IPv4, revised. IETF Request for Comments: 5944. (Obsoletes: 3344).

10. Perkins C, Johnson D \& Arkko J (2011). Mobility support in IPv6. IETF Request for Comments: 6275.

11. Perkins C (1998) Mobile IP: design principles and practices. Addison-Wesley. 275 pages.

12. Pahlavan K \& Krishnamurthy P (2002) Principles of Wireless Networks: A Unified Approach. Prentice Hall PTR, New Jersey, USA. 608 pages.

13. Sutinen T (2004) End user service quality in multi-access networks. Master's thesis, University of Oulu, Department of Electrical and Information Engineering, Finland. 80 pages.

14. Floyd S, Handley M, Padhye J \& Widmer J (2000) Equation-based congestion control for unicast applications. SIGCOMM Computer Communication Review 30(4): 43-56.

15. Tappayuthpijarn K, Liebl G, Stockhammer T \& Steinbach E (2009) Adaptive video streaming over a mobile network with TCP-friendly rate control. In: The 2009 International Conference on Wireless Communications and Mobile Computing (IWCMC '09), pp. 1325-1329. Leipzig, Germany.

16. Kofler I, Seidl J, Timmerer C, Hellwagner H, Djama I \& Ahmed T (2008) Using MPEG-21 for cross-layer multimedia content adaptation. Springer Journal on Signal, Image and Video Processing 2: 355-370. 
17. Miras D (2004) On quality aware adaptation of internet video. Ph.D. thesis, Department of Computer Science, University College London.

18. Srivastava V \& Motani M (2005) Cross-layer design: A survey and the road ahead. IEEE Communications Magazine 43(12): 112-119.

19. Huusko J, Vehkaperä J, Amon P, Lamy-Bergot C, Panza G, Peltola J \& Martini M (2007) Cross-layer architecture for scalable video transmission in wireless network. Signal Processing: Image Communication 22: 317-330.

20. Lamy-Bergot C, Fracchia R, Mazzotti M, Moretti S, Piri E, Sutinen T, Zhuo J, Vehkaperä J, Feher G, Jeney G, Panza G \& Amon P (2011) Optimisation of multimedia over wireless IP links via $\mathrm{X}$-layer design: an end-to-end transmission chain simulator. Springer Multimedia Tools and Applications 55(2): 261-288.

21. Luoto M (2008) Mobility triggering in a multiaccess environment. Master's thesis, University of Oulu, Department of Electrical and Information Engineering, Finland. 50 pages.

22. IEEE (2012). Wireless LAN medium access control (MAC) and physical layer (PHY) specifications. IEEE Std 802.11-2012.

23. IEEE (2012). MAC enhancements for robust audio video streaming. IEEE Std 802.11aa2012.

24. Halonen T, Romero J \& Melero J (2002) GSM, GPRS, and EDGE performance: Evolution towards 3G/UMTS. John Wiley \& Sons, Ltd. 585 pages.

25. Holma H \& Toskala A (eds.) (2010) WCDMA for UMTS: HSPA Evolution and LTE. John Wiley \& Sons, Ltd., 5th edition. 629 pages.

26. Holma H \& Toskala A (eds.) (2011) LTE for UMTS: Evolution to LTE-Advanced. John Wiley \& Sons, Ltd., 2nd edition. 576 pages.

27. Ekstrom H (2009) QoS control in the 3GPP evolved packet system. IEEE Communications Magazine 47(2): 76-83.

28. Andrews J, Ghosh A \& Muhamed R (2007) Fundamentals of WiMAX: Understanding broadband wireless networking. Prentice Hall. 449 pages.

29. Motorola (2007). Driving 4G: WiMAX \& LTE. Positioning paper. URI: http://www.motorola.com/web/Business/Solutions/Industry\%20Solutions/Service\%20 Providers/Wireless\%20Operators/Wireless\%20Broadband/wi4\%20WiMAX/_Document/ StaticFile/a\%20Driving_4G_WiMAX_and_LTE.pdf (Visited on 11 July 2012).

30. IEEE (2009). Air interface for broadband wireless access systems. IEEE Std 802.16-2009.

31. IEEE (2011). Air interface for broadband wireless access systems amendment 3: Advanced air interface. IEEE Std 802.16m-2011 (Amendment to IEEE Std 802.16-2009).

32. IEEE (2008). Carrier sense multiple access with collision detection (CSMA/CD) access method and physical layer specifications - section one. IEEE Std 802.3-2008.

33. Ha J, Kim J, Kim JU \& Kim SH (2009) Dynamic load balancing architecture in heterogeneous wireless network environment. In: The 9th International Conference on Communications and Information Technologies (ISCIT '09), pp. 248-253. Incheon, Korea.

34. Sankaran C (2012) Data offloading techniques in 3GPP rel-10 networks: A tutorial. IEEE Communications Magazine 50(6): 46-53.

35. Anderson S (2010) Over-the-top video. Broadcast Engineering (World Edition) 52(11): $18-19$.

36. Ott J (2006) Application protocol design considerations for a mobile internet. In: Proceedings of first ACM/IEEE international workshop on Mobility in the evolving internet architecture, MobiArch '06, pp. 75-80. San Francisco, CA, USA. 
37. Montavont N \& Noël T (2002) Handover management for mobile nodes in IPv6 networks. IEEE Communications Magazine pp. 38-43.

38. Moskowitz R \& Nikander P (2006). Host identity protocol (HIP) architecture. IETF Request for Comments: 4423.

39. Pierrel S, Jokela P, Melén J \& Slavov K (2007) A policy system for simultaneous multiaccess with host identity protocol. In: 1st IEEE Workshop on Autonomic Communication and Network Management (ACNM '07), pp. 71-77. Munich, Germany.

40. Stewart R, Xie Q, Morneault K, Sharp C, Schwarzbauer H, Taylor T, Rytina I, Kalla M, Zhang L \& Paxson V (2000). Stream control transmission protocol. IETF Request for Comments: 2960.

41. Ford A, Raiciu C, Handley M, Barre S \& Iyengar J (2011). Architectural guidelines for multipath TCP development. IETF Request for Comments: 6182.

42. Singh V, Karkkainen T, Ott J, Ahsan S \& Eggert L (2012). Multipath RTP (MPRTP). IETF Internet-Draft. URI: http://tools.ietf.org/html/draft-singh-avtcore-mprtp-05 (Visited on September 25 2012).

43. Rosenberg J, Schulzrinne H, Camarillo G, Johnston A, Peterson J, Sparks R, Handley M \& Schooler E (2002). SIP: session initiation protocol. IETF Request for Comments: 3261.

44. Wakikawa RE (2009). Multiple care-of addresses registration. IETF Request for Comments: 5648.

45. Tsirtsis G, Soliman H, Montavont N, Giaretta G \& Kuladinithi K (2011). Flow bindings in mobile IPv6 and network mobility (NEMO) basic support. IETF Request for Comments: 6089. (Updates: 5648).

46. Gundavelli, S (Ed) (2012). Flow binding support for mobile IPv4. IETF Internet-Draft. URI: http://tools.ietf.org/html/draft-ietf-mip4-multiple-tunnel-support-04 (Visited on August 10 2012).

47. IEEE (2009). Media independent handover services. IEEE Std 802.21-2009.

48. Piri E \& Pentikousis K (2009) IEEE 802.21: Media-independent handover services. Internet Protocol Journal 12(2).

49. Mäkelä J \& Pentikousis K (2007) Trigger management mechanisms. In: The 2nd international symposium on wireless pervasive computing (ISWPC '07), pp. 378-383. San Juan, Puerto Rico.

50. Räisänen V (2003) Implementing service quality in IP networks. John Wiley \& Sons, Ltd. 325 pages.

51. Wright D (2007) Maintaining QoS during handover among multiple wireless access technologies. In: Management of Mobile Business (ICMB '07), p. 10. Toronto, Canada.

52. Marques V, Aguiar R, Garcia C, Moreno J, Beaujean C, Melin E \& Liebsch M (2003) An IP-based QoS architecture for 4G operator scenarios. IEEE Wireless Communications 10(3): 54-62.

53. Pliakas T, Kormentzas G \& Tsekeridou S (2007) Joint scalable video coding and packet prioritisation for video streaming over IP/802.11e heterogeneous networks. In: The 3rd International Conference on Mobile Multimedia Communications (Mobimedia '07), pp. 31:1-31:6. Nafpakos, Greece.

54. Gundavelli, S (Ed) (2008). Proxy mobile IPv6. IETF Request for Comments: 5213.

55. Makela A \& Korhonen J (2012). Home agent-assisted route optimization between mobile IPv4 networks. IETF Request for Comments: 6521. 
56. Montenegro, G (Ed) (2001). Reverse tunneling for mobile IP revised. IETF Request for Comments: 3024 .

57. Perkins CE \& Johnson DB (1998) Route optimization for mobile IP. Cluster Computing, Special Issue on Mobile Computing 1(2): 161-176.

58. Kassar M, Kervella B \& Pujolle G (2008) An overview of vertical handover decision strategies in heterogeneous wireless networks. Computer Communications 31(10): 26072620.

59. Zhang W, Jaehnert J \& Dolzer K (2003) Design and evaluation of a handover decision strategy for 4th generation mobile networks. In: The 57th IEEE Semiannual Vehicular Technology Conference (VTC 2003-Spring), volume 3, pp. 1969-1973. Jeju, Korea.

60. Wu D, Hou YT \& Zhang YQ (2000) Transporting real-time video over the internet: challenges and approaches. Proceedings of the IEEE 88(12): 1855-1877.

61. Eleftheriadis A, Eleftheriadis R \& Anastassiou D (1995) Meeting arbitrary QoS constraints using dynamic rate shaping of coded digital video. In: Fifth International Workshop on Network and Operating System Support for Digital Audio and Video (NOSSDAV '95), pp. 95-106. Durham, NH, USA.

62. Stockhammer T (2011) Dynamic adaptive streaming over HTTP: standards and design principles. In: Proceedings of the second annual ACM conference on multimedia systems (MMSys '11), pp. 133-144. San Jose, CA, USA.

63. Schierl T, Grüneberg K \& Wiegand T (2009) Scalable video coding over RTP and MPEG-2 transport stream in broadcast and IPTV channels. IEEE Wireless Communications Magazine 16(5): 64-71.

64. Wenger S, Wang Y \& Hannuksela M (2006) RTP payload format for H.264/SVC scalable video coding. Journal of Zhejiang University SCIENCE A 7(5): 657-667.

65. Ransburg M, Graciá E, Sutinen T, Ortíz J, Sablatschan M \& Hellwagner H (2012) Scalable video coding impact on networks. In: Mobile Multimedia Communications, volume 77 of Lecture Notes of the Institute for Computer Sciences, Social Informatics and Telecommunications Engineering, pp. 571-581. Springer.

66. Frederick R, Schulzrinne H, Casner S \& Jacobson V (2003). RTP: A transport protocol for real-time applications. IETF Request for Comments: 3550 .

67. Lanphier R, Schulzrinne H \& Rao A (1998). Real time streaming protocol (RTSP). IETF Request for Comments: 2326.

68. Schierl T \& Wenger S (2009). Signaling media decoding dependency in the session description protocol (SDP). IETF Request for Comments: 5583.

69. Schwarz H, Marpe D \& Wiegand T (2007) Overview of the scalable video coding extension of the H.264/AVC standard. IEEE Transactions on Circuits and Systems for Video Technology 17: 1103-1120.

70. ITU-T (2007) H.264: Advanced video coding for generic audiovisual services.

71. Kofler I, Kuschnig R \& Hellwagner H (2009) Improving IPTV services by H.264/SVC adaptation and traffic control. In: IEEE International Symposium on Broadband Multimedia Systems and Broadcasting (BMSB '09), pp. 1-6. Bilbao, Spain.

72. Wenger S, Wang YK, Schierl T \& Eleftheriadis A (2011). RTP payload format for scalable video coding. IETF Request for Comments: 6190.

73. Kofler I, Prangl M, Kuschnig R \& Hellwagner H (2008) An H.264/SVC-based adaptation proxy on a WiFi router. In: 18th International Workshop on Network and Operating Systems Support for Digital Audio and Video (NOSSDAV '08), pp. 63-68. Braunschweig, Germany. 
74. Schierl T, de la Fuente Y, Hellge C \& Wiegand T (2009) Decoding order recovery for multi flow transmission of scalable video coding (SVC) over mobile IP channels. In: The 5th International Mobile Multimedia Communications Conference (Mobimedia '09), pp. 12:1-12:4. London, United Kingdom.

75. Chang SF \& Vetro A (2005) Video adaptation: Concepts, technologies, and open issues. Proceedings of the IEEE 93(1): 148-158.

76. Bouch A, Kuchinsky A \& Bhatti N (2000) Quality is in the eye of the beholder: Meeting users' requirements for internet quality of service. CHI Letters 2(1): 297-304.

77. Zink M, Schmitt J \& Steinmetz R (2005) Layer-encoded video in scalable adaptive streaming. IEEE Transactions on Multimedia 7(1): 75-84.

78. Ni Z, Chen Z \& Ngan K (2005) Signal processing: Image communication. IEEE Transactions on Multimedia 20(3): 277-293.

79. Uitto M \& Vehkaperä J (2009) Spatial enhancement layer utilisation for SVC in base layer error concealment. In: The 5th International Mobile Multimedia Communications Conference (Mobimedia '09), pp. 10:1-10:7. London, UK.

80. Daronco LC, Roesler V, de Lima JV \& Balbinot R (2011) Quality analysis of scalable video coding on unstable transmissions. Multimedia Tools and Applications p. 27.

81. Handley M, Floyd S, Padhye J \& Widmer J (2003). TCP friendly rate control (TFRC): Protocol specification. IETF Request for Comments: 3448.

82. Srivastava V \& Motani M (2005) Cross-layer design: A survey and the road ahead. IEEE Communications Magazine 43(12): 112-119.

83. Fortuna C \& Mohorcic M (2009) Trends in the development of communication networks: Cognitive networks. Computer Networks 53(9): 1354-1376.

84. Kawadia V \& Kumar PR (2005) A cautionary perspective on cross-layer design. IEEE Wireless Communications 12(1): 3-11.

85. Daniel L \& Kojo M (2008) Employing cross-layer assisted TCP algorithms to improve TCP performance with vertical handoffs. International Journal of Communication Networks and Distributed Systems 1(4/5/6): 433-465.

86. Gurtov A \& Korhonen J (2004) Effect of vertical handovers on performance of TCP-friendly rate control. SIGMOBILE Mobile Computing and Communications Review 8: 73-87.

87. Fallah Y, Nasiopoulos P \& Alnuweiri H (2007) Scheduled and contention access transmission of partitioned H.264 video over WLANs. In: IEEE Global Communications Conference (Globecom '07), pp. 2134-2139. Washington DC, USA.

88. Chilamkurti N, Zeadally S, Soni R \& Giambene G (2010) Wireless multimedia delivery over IEEE 802.11e with cross-layer optimization techniques. Multimedia Tools and Applications 47: 189-205.

89. Ramakrishnan K, Floyd S \& Black D (2001). The addition of explicit congestion notification (ECN) to IP applications. IETF Request for Comments: 3168.

90. Eardley, P (Ed) (2009). Pre-congestion notification (PCN) architecture. IETF Request for Comments: 5559.

91. Menth M, Briscoe B \& Tsou T (2012) Precongestion notification: new QoS support for differentiated services IP networks. IEEE Communications Magazine 50(3): 94-103.

92. Mäkelä J, Pentikousis K \& Kyllönen V (2009) Mobility trigger management: Implementation and evaluation. International Journal of Communications, Network and System Sciences 2(3): 211-221. 
93. Dousson C, Pentikousis K, Sutinen T \& Mäkelä J (2007) Chronicle recognition for mobility management triggers. In: The 12th IEEE Symposium on Computers and Communications (ISCC 2007), pp. 305-310. Aveiro, Portugal.

94. Pääkkönen P, Salmela P, Aguero R \& Choque J (2007) An integrated ambient networks prototype. In: The 15th International Conference on Software, Telecommunications and Computer Networks (SoftCOM '07), pp. 1-5. Split - Dubrovnik, Croatia.

95. Mäkelä J, Luoto M, Sutinen T \& Pentikousis K (2011) Distributed information service architecture for overlapping multiaccess networks. Multimedia Tools and Applications 55(2): 289-306.

96. Kliazovich D, Sutinen T, Kokkoniemi-Tarkkanen H, Mäkelä J \& Horsmanheimo S (2011) Hierarchical management architecture for multi-access networks. In: IEEE Global Communications Conference (Globecom '11), pp. 1-6. Houston, TX, USA.

97. Piri E, Sutinen T \& Vehkaperä J (2009) Cross-layer architecture for adaptive realtime multimedia in heterogeneous network environment. In: European Wireless, pp. 293-297. Aalborg, Denmark.

98. Hämäläinen S, Sannek H \& Sartori C (eds.) (2012) LTE Self-organizing Networks (SON). John Wiley \& Sons, Ltd., 1st edition. 398 pages.

99. Horsmanheimo S, Eskelinen J \& Kokkoniemi-Tarkkanen H (2010) NES - Network Expert System for Heterogeneous Networks. In: The 17th International Conference on Telecommunications (ICT '10), pp. 680-685. Doha, Qatar.

100. Friedman T, Caceres R \& Clark A (2003). RTP control protocol extended reports (RTCP XR). IETF Request for Comments: 3611.

101. Ott J \& Perkins C (2010). Guidelines for extending the RTP control protocol (RTCP). IETF Request for Comments: 5968.

102. Liebl G, Schierl T, Wiegand T \& Stockhammer T (2006) Advanced wireless multiuser video streaming using the scalable video coding extensions of H.264/MPEG-4-AVC. In: IEEE International Conference on Multimedia and Expo (ICME '06), pp. 625-628. Toronto, Canada.

103. Juan HH, Huang HC, Huang C \& Chiang T (2010) Cross-layer mobile WiMAX MAC designs for the H.264/AVC scalable video coding. Springer Wireless Networks 16: 113-123.

104. Bianchi G, Detti A, Loreti P, Pisa C, Proto F, Kellerer W, Thakolsri S \& Widmer J (2009) Application-aware H.264 scalable video coding delivery over wireless LAN: experimental assessment. In: Second International Workshop on Cross Layer Design (IWCLD '09), pp. 1-6. Palma de Mallorca, Spain.

105. Chen HL, Lee PC \& Hu SH (2008) Improving scalable video transmission over IP/802.11e through a cross-layer architecture. In: The 4th International Conference on Wireless and Mobile Communications (ICWMC '08), pp. 241-246. Athens, Greece.

106. Haratcherev I, Taal J, Langendoen K, Lagendijk R \& Sips H (2006) Optimized video streaming over 802.11 by cross-layer signaling. IEEE Communications Magazine 44(1): $115-121$.

107. Huang CW, Loiacono M, Rosca J \& Hwang JN (2009) Airtime fair distributed cross-layer congestion control for real-time video over WLAN. IEEE Transactions on Circuits and Systems for Video Technology 19(2): 1158-1168.

108. Winkler S \& Mohandas P (2008) The evolution of video quality measurement: From PSNR to hybrid metrics. IEEE Transactions on Broadcasting 54(3): 660-668. 
109. Åhlund C, Brännström R \& Zaslavsky A (2005) M-MIP: Extended mobile IP to maintain multiple connections to overlapping wireless access networks. In: Lecture Notes in Computer Science, volume 3420, pp. 204-213.

110. Prokkola J, Hanski M, Jurvansuu M \& Immonen M (2007) Measuring WCDMA and HSDPA delay characteristics with QoSMeT. In: IEEE International Conference on Communications (ICC '07), pp. 492-498. Glasgow, Scotland.

111. Ciubotaru B \& Muntean GM (2009) SASHA - a quality-oriented handover algorithm for multimedia content delivery to mobile users. IEEE Transactions on Broadcasting 55: 437.

112. Andersson K \& Åhlund C (2011) Optimized access network selection in a combined WLAN/LTE environment. Wireless Personal Communications 61(4): 739-751.

113. Freris NM, Hsu CH, Zhu X \& Singh JP (2010) Resource allocation for multihomed scalable video streaming to multiple clients. In: The 2010 IEEE International Symposium on Multimedia (ISM '10), pp. 9-16. Taichung, Taiwan.

114. Vesanto J \& Alhoniemi E (2000) Clustering of the self-organizing map. IEEE Transactions on Neural Networks 11(3): 586-600. 


\section{Original papers}

I Sutinen T \& Huusko J (2010) MAC layer QoS architecture for optimized and fair transmission of scalable video. In IEEE Consumer Communications and Networking Conference (CCNC '10), Las Vegas, Nevada, USA: 421-425.

II Sutinen T, Vehkaperä J, Piri E \& Uitto M (2012) Towards ubiquitous video services through scalable video coding and cross-layer optimization. EURASIP Journal on Wireless Communications and Networking 2012:25.

III Luoto M \& Sutinen T (2008) Cross-layer enhanced mobility management in heterogeneous networks. In IEEE International Conference on Communications (ICC '08), Beijing, China: 2277-2281.

IV Sutinen T \& Rivas H (2011) Multi-interface extension to a scalable video streaming architecture. Journal of Communications 6(9): 700-710.

V Ojanperä T, Luoto M, Ortiz J \& Myllyniemi M (2012) Integrating adaptive video streaming service with multi-access network management. Mobile Networks and Applications 17(4): 492-505.

Reprinted with kind permissions from IEEE (I \& III), Springer Science and Business Media (II \& V), and Academy Publisher (IV).

Original publications are not included in the electronic version of the dissertation. 


\section{ACTA UNIVERSITATIS OULUENSIS \\ SERIES C TECHNICA}

436. Ossiannilsson, Ebba (20I2) Benchmarking e-learning in higher education : lessons learned from international projects

437. Pouttu, Ari (20/2) Performance Analysis of mMCSK-mMFSK modulation variants with comparative discussion

438. Kupiainen, Laura (20I2) Dilute acid catalysed hydrolysis of cellulose - extension to formic acid

439. Kurtti, Sami (20I2) Integrated receiver channel and timing discrimination circuits for a pulsed time-of-flight laser rangefinder

440. Distanont, Anyanitha (2012) Knowledge transfer in requirements engineering in collaborative product development

44I. Keskinarkaus, Anja (20I2) Digital watermarking techniques for printed images

442. Sorsa, Aki (20/3) Prediction of material properties based on non-destructive Barkhausen noise measurement

443. Sangi, Pekka (2013) Object motion estimation using block matching with uncertainty analysis

444. Duan, Guoyong (2013) Three-dimensional effects and surface breakdown addressing efficiency and reliability problems in avalanche bipolar junction transistors

445. Hirvonen-Kantola, Sari (2013) Eheyttäminen, kestävä kehittely ja yhteyttäminen : integroivaa kaupunkikehitystyötä Vantaalla

446. Hannu, Jari (20/3) Embedded mixed-signal testing on board and system level

447. Sonkki, Marko (20/3) Wideband and multi-element antennas for mobile applications

448. Saarinen, Tuomas (20/3) Temporal and spatial variation in the status of acid rivers and potential prevention methods of AS soil-related leaching in peatland forestry

449. Pirinen, Rauno (2013) Towards regional development by Higher Education Institutions : an empirical study of a University of Applied Sciences

450. Mäkinen, Liisa (2013) Improvement of resource efficiency in deinked pulp mill

45I. Guo, Yimo (2013) Image and video analysis by local descriptors and deformable image registration

Book orders:

Granum: Virtual book store http://granum.uta.fi/granum/ 


\section{A C TA UN IVER SITATIS O ULUENSIS}

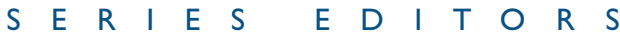

\section{SCIENTIAE RERUM NATURALIUM}

Senior Assistant Jorma Arhippainen

\section{HUMANIORA}

University Lecturer Santeri Palviainen

\section{TECHNICA}

Docent Hannu Heusala

\section{MEDICA}

Professor Olli Vuolteenaho

\section{SCIENTIAE RERUM SOCIALIUM}

University Lecturer Hannu Heikkinen

\section{SCRIPTA ACADEMICA}

Director Sinikka Eskelinen

\section{OECONOMICA}

$$
\text { Professor Jari Juga }
$$

EDITOR IN CHIEF

Professor Olli Vuolteenaho

PUBLICATIONS EDITOR

Publications Editor Kirsti Nurkkala

ISBN 978-952-62-0I50-4 (Paperback)

ISBN 978-952-62-0I5I-I (PDF)

ISSN 0355-32I 3 (Print) 